UCRL-ID-133846

\title{
Fracture Characterization of the Large-Block Test, Fran Ridge, Yucca Mountain, Nevada
}

Jeffrey L. Wagoner

May 1999

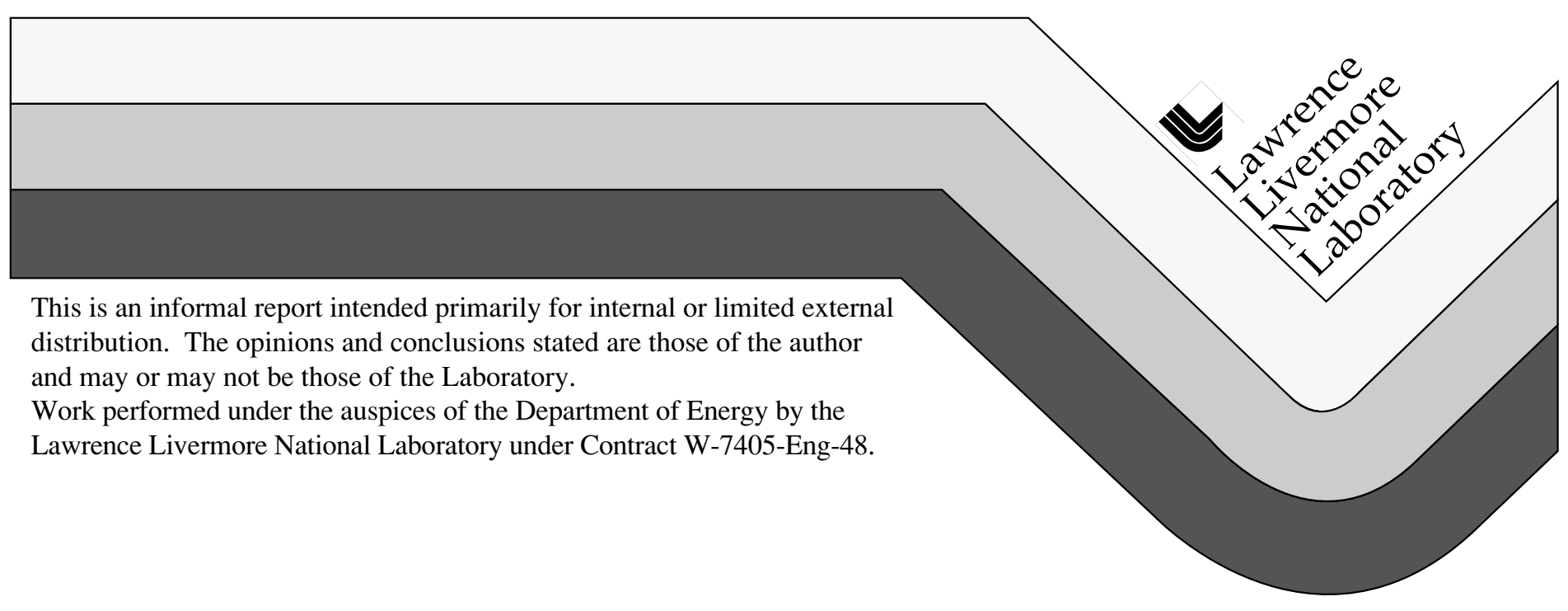




\section{DISCLAIMER}

This document was prepared as an account of work sponsored by an agency of the United States Government. Neither the United States Government nor the University of California nor any of their employees, makes any warranty, express or implied, or assumes any legal liability or responsibility for the accuracy, completeness, or usefulness of any information, apparatus, product, or process disclosed, or represents that its use would not infringe privately owned rights. Reference herein to any specific commercial product, process, or service by trade name, trademark, manufacturer, or otherwise, does not necessarily constitute or imply its endorsement, recommendation, or favoring by the United States Government or the University of California. The views and opinions of authors expressed herein do not necessarily state or reflect those of the United States Government or the University of California, and shall not be used for advertising or product endorsement purposes.

This report has been reproduced

directly from the best available copy.

Available to DOE and DOE contractors from the

Office of Scientific and Technical Information

P.O. Box 62, Oak Ridge, TN 37831

Prices available from (615) 576-8401, FTS 626-8401

Available to the public from the

National Technical Information Service

U.S. Department of Commerce

5285 Port Royal Rd.,

Springfield, VA 22161 


\section{Contents}

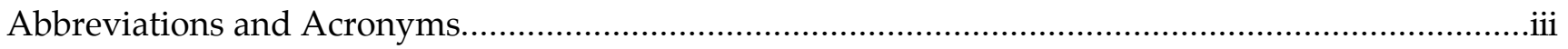

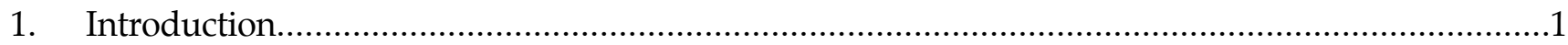

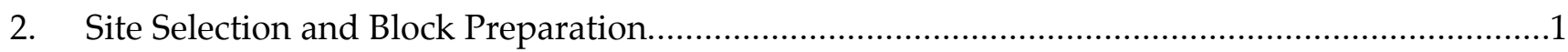

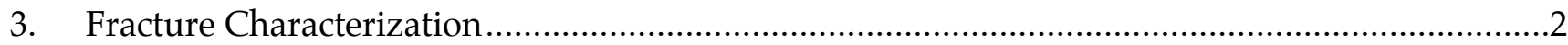

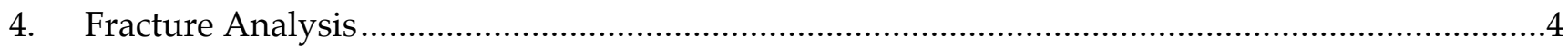

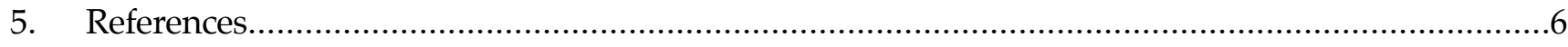

Appendix A. Structures Documented in the Borehole Videos................................................. A-1

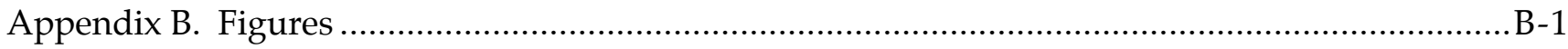

\section{Tables}

Table 1. Number of fractures per each length interval and the percentage of fractures occurring in each interval (fractures mapped on all five sides) ......................

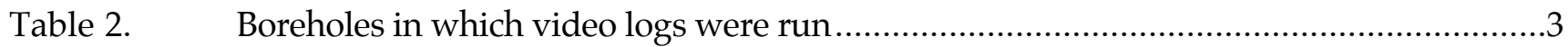

Table 3. All major fracture planes that have been modeled for the LBT................................4

\section{Figures}

Figure 1. Mapped surface fractures on the large block .................................................

Figure 2. All boreholes in the large block .................................................................. $\quad$ -

Figure 3. Equal-area diagram of poles to major fractures mapped for the LBT ...................B-3

Figure 4. $\quad 3-\mathrm{D}$ depiction of the major mappable fractures cutting the large block ................... B-4

Figure 5. $\quad$ Fracture system \#1 contains 3 fractures that strike N50E and

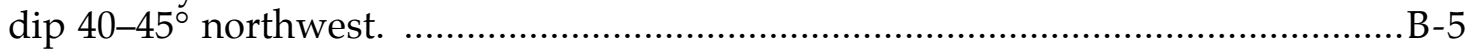

Figure 6. Fracture system \#2 contains 2 fractures that strike N30-40W and dip

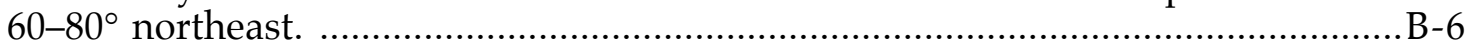

Figure 7. Fracture system \#3 contains 6 subhorizontal fractures. Fracture LBT1 (green) is the largest, most significant fracture mapped in the block.

Figure 8. Fracture system \#4 contains 13 mapped fractures and is the dominant system in the block. This system strikes northwest and dips toward the southwest.

Figure 9. Fracture system \#5 contains 4 approximately vertical fractures that strike east-west.

Figure 10. Fracture system \#6 contains 2 mappable fractures that strike northeast and dip to the southeast. B-10 
Figure 11. Analysis of fractures mapped after the top surface was leveled above the large block

Figure 12. Equal-area contour diagram of poles to 90 fractures mapped in the Climax Stock granite (concentrations in percentage points within one percent area counting circle).

Figure 13. Fractures mapped at the Fran Ridge site prior to construction of the LBT (square): mapped LBT fracture systems were projected to this surface (color coded).

Figure 14. Mapped fractures on top of the LBT

Figure 15. Mapped fractures on the west face of the LBT. B-15

Figure 16. Mapped fractures on the south face of the LBT B-16

Figure 17. Mapped fractures on the north face of the LBT. B-17

Figure 18. Mapped fractures on the east face of the LBT. B-18 


\title{
Abbreviations and Acronyms
}

\author{
3-D three-dimensional \\ DOE U.S. Department of Energy \\ LBT Large Block Test
}

Fracture Characterization of the Large-Block Test 



\section{Fracture Characterization of the Large Block Test, Fran Ridge, Yucca Mountain, Nevada}

\section{Introduction}

The U.S. Department of Energy (DOE) is investigating the suitability of Yucca Mountain as a potential site for the nation's first high-level nuclear waste repository. The site is located about $120 \mathrm{~km}$ northwest of Las Vegas, Nevada, at the Nevada Test Site. Favorable aspects of Yucca Mountain as a potential repository site include its arid nature and the sorptive properties of the rock materials. The arid environment results in unsaturated conditions at the potential emplacement horizon, which is the Topopah Spring tuff of the Paintbrush Group.

The Large Block Test (LBT) was designed to be one of a series of tests at different scales and conditions that assist in defining the physical processes that need to be considered in models of a potential repository in Yucca Mountain. The LBT is a critical test because it is of sufficient size to incorporate a fracture system that is representative of the distribution of fracture dimensions and characteristics-with the exception of major structures, such as faults - that would likely be present in a repository. The LBT location was chosen to include large, through-going fractures as well as small, healed fractures that are of limited extent. The LBT location also includes a variety of fracture sizes, connectivities, and characteristics that fall between the bounds of the large and very small fractures. The LBT allows for boundary controls and monitoring that are somewhat similar to those typical of laboratory studies, and it allows for three-dimensional (3-D) characterization and monitoring. The unique combination of size with boundary controls of the LBT allows processes to be evaluated and models to be tested more completely than in tests of any other scale (Wilder et al. 1997, Section 1).

\section{Site Selection and Block Preparation}

Yucca Mountain lies within the southern part of the Great Basin subprovince of the Basin and Range province in southwestern Nevada, at the southwest corner of the Nevada Test Site. Yucca Mountain is characterized by a series of north-trending, eastward-dipping elongated structural blocks that have been pulled apart and tilted along normal faults. Fran Ridge is located on the east side of Yucca Mountain.

The site at Fran Ridge was selected for the LBT because of its desirable rock type, fracture characteristics, and accessibility. The general location was stripped of vegetation and soil to expose the bedrock. All significant fractures were then mapped in the area. Based on this mapping, it was judged that the general site was adequate for the LBT, specifically that the rock fracturing and matrix block sizes were consistent with what was anticipated to exist at the potential Yucca Mountain repository site (Wilder 1993). Vertical instrumentation holes within the block were drilled and cored before the sawing and excavation of the block A belt saw was used to saw four vertical slots that formed the boundary of the large block. By this excavation process, a block of Topopah Spring tuff measuring $3 \times 3 \times 4.5 \mathrm{~m}$ was 
isolated at Fran Ridge. After block excavation was completed and the surfaces were mapped, horizontal boreholes were located (vertical holes had been drilled prior to excavation) for emplacing instrumentation and heaters.

\section{Fracture Characterization}

Characterization of the block began with mapping and analysis of the distribution of fractures. Fractures were carefully mapped using a 1-ft x 1-ft grid system on all 4 vertical sides of the block. Fracture mapping started on September 19, 1994, and was completed on October 6, 1994. Each fracture was assigned a unique number on each side of the block, and fracture attitudes were measured where possible. Fracture surface roughness was not recorded. More than 2400 individual fractures were mapped. The range and distribution of fracture lengths are recorded in Table 1. The fracture locations were digitized, and fracture segment nodes were assigned $x-y-z$ values. These scattered data points were then input into a 3-D modeling code (Earthvision ${ }^{\mathrm{TM}}$ ). The resulting surface fracture distribution is shown in Figure 1, which lists fractures ordered by length and shows that most of the fractures are less than $1 \mathrm{~m}$ long.

Table 1. Number of fractures per each length interval and the percentage of fractures occurring in each interval (fractures mapped on all five sides)

\begin{tabular}{cccc}
\hline $\begin{array}{c}\text { Number of } \\
\text { Fractures }\end{array}$ & Length $(\mathbf{m})$ & Percentage & $\begin{array}{c}\text { Cumulative } \\
\text { Percentage }\end{array}$ \\
\hline 1044 & $0.0-.15$ & 43.41 & 43.41 \\
631 & $.15-.30$ & 26.24 & 69.65 \\
251 & $.30-.46$ & 10.44 & 80.08 \\
130 & $.46-.61$ & 5.41 & 85.49 \\
118 & $.61-.76$ & 4.91 & 90.40 \\
59 & $.76-.91$ & 2.45 & 92.85 \\
35 & $.91-1.07$ & 1.46 & 94.30 \\
32 & $1.07-1.22$ & 1.33 & 95.63 \\
24 & $1.22-1.37$ & 1.00 & 96.63 \\
13 & $1.37-1.52$ & 0.54 & 97.17 \\
8 & $1.52-1.68$ & 0.33 & 97.51 \\
5 & $1.68-1.83$ & 0.21 & 97.71 \\
8 & $1.83-1.98$ & 0.33 & 98.05 \\
6 & $1.98-2.13$ & 0.25 & 98.30 \\
6 & $2.13-2.29$ & 0.25 & 98.54 \\
3 & $2.29-2.44$ & 0.12 & 98.67 \\
3 & $2.44-2.59$ & 0.12 & 98.79 \\
2 & $2.59-2.74$ & 0.08 & 98.88 \\
5 & $2.74-2.90$ & 0.21 & 99.09 \\
2 & $2.90-3.05$ & 0.08 & 99.17 \\
& & &
\end{tabular}




\begin{tabular}{cccc}
\hline $\begin{array}{c}\text { Number of } \\
\text { Fractures }\end{array}$ & Length $(\mathbf{m})$ & Percentage & $\begin{array}{c}\text { Cumulative } \\
\text { Percentage }\end{array}$ \\
\hline 8 & $3.05-3.20$ & 0.33 & 99.50 \\
0 & $3.20-3.35$ & 0.00 & 99.50 \\
0 & $3.35-3.51$ & 0.00 & 99.50 \\
1 & $3.51-3.66$ & 0.04 & 99.54 \\
0 & $3.66-3.81$ & 0.00 & 99.54 \\
0 & $3.81-3.96$ & 0.00 & 99.54 \\
2 & $3.96-4.11$ & 0.08 & 99.63 \\
0 & $4.11-4.27$ & 0.00 & 99.63 \\
3 & $4.27-4.42$ & 0.12 & 99.75 \\
0 & $4.42-4.57$ & 0.00 & 99.75 \\
2 & $4.57-4.72$ & 0.08 & 99.83 \\
3 & $4.72-4.88$ & 0.12 & 99.96 \\
0 & $4.88-5.03$ & 0.00 & 99.96 \\
1 & $5.03-5.18$ & 0.04 & 100.00 \\
\hline
\end{tabular}

Numerous boreholes were drilled in the large block for installation of monitoring instrumentation and observation. Figure 2 shows the location of those boreholes. The angled boreholes (color-coded yellow) are the post-test boreholes.

Fracture data were also collected from the borehole video logs of these holes. Information on the core is not included in this document. Detailed fracture information from the video logs is available in Appendix A. Seventy-one boreholes were videotaped. In that process, a measuring tape was placed in the borehole for location purposes. In the case of the vertical boreholes, the tape was hung along the north side of the hole, which provided an orientation in the borehole. Fractures were logged and described in the boreholes listed in Table 2. The depths at which the fracture enters and exits the borehole were recorded as were the strike, dip, dip direction, aperture, and magnitude of the features. Figure 2 shows the location of the boreholes in the large block. The angled boreholes (color-coded yellow) are the post-test boreholes.

Table 2. Boreholes in which video logs were run

\begin{tabular}{ll}
\hline Vertical boreholes drilled from the top of LBT & E1, E2, E3, E4, E5, E6, E7, E8, E9, E10, \\
& N1, N2, N3, N4, N5, N6, N7 \\
Horizontal boreholes drilled from the east side of LBT & EH1, EH2, EH3, EH4, EH5, \\
& E03 \\
Horizontal boreholes drilled from the west side of LBT & W05, \\
& WH1, WH2, \\
& WM1, WM2, WM3, \\
& WN2, WN3, WN4, \\
& WT1, WT2, WT3 \\
Horizontal boreholes drilled from the west side of LBT & N01, N02, \\
& NH1, NM1, NM2, NM3, \\
& NN1, NN2, NN3, NN4, NN5, NN6, \\
& NT1, NT2, NT3, NT4
\end{tabular}


Post-test bore holes drilled from the north side of LBT

Post-test bore holes drilled from the west side of LBT

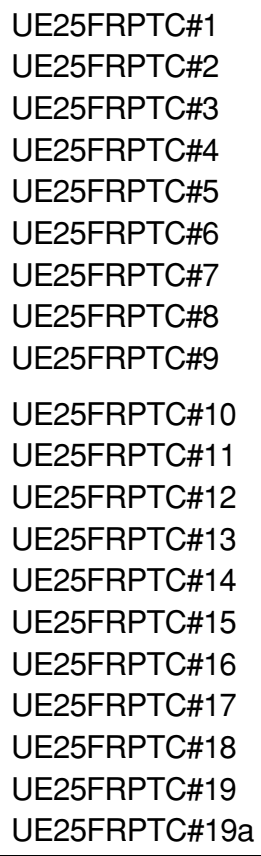

\section{Fracture Analysis}

The goal of the fracture analysis is to identify and model the major through-going structures that penetrate the LBT. This involved correlating the surface fracture traces with the location of fractures intersecting the boreholes. Correlation of the borehole fractures with the surface fractures is confirmed by the location and the strike and dip of the fracture as measured in the video log.

Figure 3 is an equal-area diagram of pole to the major fractures that have been defined for the LBT. These fractures are defined by the surface mapping of the LBT and by the video mapping of the boreholes. Table 3 lists the major mappable fractures that have been modeled in the LBT. The individual modeled fractures are grouped into six fracture systems based on similarity in strike and dip. Figure 4 is a 3-D perspective of these six systems cutting the block.

Table 3. All major fracture planes that have been modeled for the LBT

These fractures are defined by the surface mapping of the LBT and by the video mapping of the boreholes.

\begin{tabular}{|c|c|c|c|c|}
\hline Number & Strike & Dip & System & Number \\
\hline LBT21 & N32E & 82NW & & 1 \\
\hline LBT11 & N49E & $43 N W$ & & 1 \\
\hline LBT22 & N52E & $58 \mathrm{NW}$ & & 1 \\
\hline LBT2 & N30W & $78 N E$ & & 2 \\
\hline LBT15 & N37W & 62NE & & 2 \\
\hline LBT14 & N26E & 01SE & & 3 \\
\hline
\end{tabular}




\begin{tabular}{cccc}
\hline Number & Strike & Dip & System \\
\hline NBT1 & N36W & 11NE & 3 \\
LBT30 & N53W & 09SW & 3 \\
LBT32 & N68W & 13NE & 3 \\
LBT31 & N70W & 09NE & 3 \\
LBT33 & N78W & 23NE & 3 \\
LBT38 & N03W & $89 S W$ & 4 \\
LBT12 & N04W & $89 S W$ & 4 \\
LBT16 & N05W & $89 S W$ & 4 \\
LBT13 & N10W & $87 S W$ & 4 \\
LBT37 & N11W & $88 S W$ & 4 \\
LBT42 & N16W & $80 S W$ & 4 \\
LBT36 & N17W & $87 S W$ & 4 \\
LBT41 & N18W & $81 S W$ & 4 \\
LBT20 & N18W & $87 S W$ & 4 \\
LBT35 & N18W & $87 S W$ & 4 \\
LBT6 & N22W & $78 S W$ & 4 \\
LBT40 & N25W & $80 S W$ & 4 \\
LBT5 & N34W & $77 S W$ & 4 \\
LBT34 & N70W & $80 S W$ & 5 \\
LBT8 & N78W & $79 S W$ & 5 \\
LBT39 & N78W & $87 N E$ & 5 \\
LBT3 & N89E & 90 & 5 \\
LBT4 & N46E & $87 S E$ & 6 \\
\hline
\end{tabular}

Fracture system \#1 is defined by three major fractures called LBT11, LBT21, and LBT22. This system occurs on the south, east, and north sides of the large block and strikes N50E then dips $40-45^{\circ}$ to the northwest (Figure 5). System \#2 consists of two major mapped fractures called LBT2 and LBT15. These fractures strike N30-40W and dip $60-80^{\circ}$ to the northeast. The two fractures are mapped on the south and west sides of the large block (Figure 6).

Fracture system \#3 contains six major mappable fractures. The fractures making up this system are LBT14, LBT30, LBT31, LBT32, and LBT33. LBT1 is by far the most significant fracture in the LBT. It completely cuts through the block and is identified in all vertical boreholes drilled from the top of the large block. As seen in the borehole videos, this feature generally has a wide aperture, common secondary mineralization, and, locally, alteration halos. These fractures are subhorizontal and have strikes that are generally northwest and dip mainly $20^{\circ}$ toward the southwest (Figure 7). These fractures occur on all four sides of the large block. The dip direction of these fractures is similar to the topographic slope of this part of Fran Ridge.

Fracture system \#4 contains the greatest number of fractures. This system is defined by 13 major mappable fractures that penetrate the large block. The fracture attitudes range from N03 to $34 \mathrm{~W}$, dipping $77-89^{\circ}$ to the southwest. This system is present on all sides of the LBT 
and contains a large number of subordinate, associated fractures with similar attitudes (Figure 8). Fracture system \#5 contains three major mappable fractures that have a general east-west strike and near vertical dips (generally 80-90 ${ }^{\circ}$ (Figure 9). Fracture system \#6 is defined by two mappable fractures, LBT4 and LBT23. The fractures strike northeast with near-vertical dips (Figure 10).

Figure 11 is an equal-area net of poles to the major fractures that were mapped on top of the large block prior to construction of the LBT. The steeply dipping fractures correlate well with Figure 3, but the subhorizontal fractures are missing. These subhorizontal fractures were not observed in the initial mapping because of the shallow dip of the structures and probable subtle expression at the surface of the ground. This fracture pattern, including the subhorizontal fracture system, compares reasonably well with the fractures and faults that were mapped in the Climax Stock (Figure 12), located approximately $50 \mathrm{~km}$ northeast of Fran Ridge (Thorpe and Springer 1981, p. 14, Figure 6).

The fracture map of the ground surface above the area of the LBT prior to construction of the block is shown in Figure 13. The highlighted fractures have been identified in the large block and then projected to the land surface. Five of the six fracture systems have been identified; the subhorizontal fracture system \#3 is not identified on this map. Identification and correlation of the fractures was done by projecting the measured attitudes of the LBT fractures up to the land surface. The gray fractures in Figure 13 were not correlated with specific fracture surfaces in the LBT. These fractures were also not assigned to a specific fracture system because attitude data for them were not available.

Figure 14 through Figure 18 are two-dimensional presentations of all fractures mapped on the five surfaces of the LBT. The fracture systems are color-coded for increased visual discrimination. The gray fractures in Figues 14 through 18 were not assigned to a specific fracture system.

\section{Summary}

This data report documents fractures in the Large Block Test at Fran Ridge, Yucca Mountain. More than 2400 individual fractures were mapped on the surface of the block, while more than 1100 fractures were documented in video logs of 71 boreholes. Individual fractures less than $.76 \mathrm{~m}$ long are the most common on the surface of the block. The major fractures were modeled as 3-D surfaces using the surface and the video fracture data. Six through-going fracture systems were identified within the block. These systems were defined by the major fractures that have similar strike and dip directions. These fracture data will be incorporated into the evaluation of fluid flow during the heating and cooling phases of the Large Block Test.

\section{References}

Thorpe, R., and Springer, J. 1981. Fracture Mapping for Radionuclide Migration Studies in the Climax Granite. UCID19081. Livermore, California: Lawrence Livermore National Laboratory. 
Wilder, D.G. 1993. Preliminary Near-Field Environment Report, Volume II: Scientific Overview of the Near-Field Environment and Phenomenon. UCRL-LR-107476, Vol. II. Livermore, California: Lawrence Livermore National Laboratory. NNA.19920501.0002.

Wilder, D.G., Lin, W., Blair, S.C., Buscheck, T.A., Carlson, R.C., Lee, K., Meike, A., Ramirez, A.L., Wagoner, J.L., and Wang, J.S.Y. 1997. Large Block Test Status Report. UCRL-ID-128776. Livermore, California: Lawrence Livermore National Laboratory. 234859. 



\section{Appendix A Structures Documented in the Borehole Videos}





\section{Structures Documented in the Borehole Videos}

\begin{tabular}{|c|c|c|c|c|c|c|c|c|}
\hline Hole & $\begin{array}{l}\text { Surface } \\
\text { Feature }\end{array}$ & $\begin{array}{c}\text { Model } \\
\text { East }\end{array}$ & $\begin{array}{l}\text { Model } \\
\text { North }\end{array}$ & $\begin{array}{l}\text { Model } \\
\text { Depth }\end{array}$ & $\begin{array}{l}\text { Strike } \\
\text { Direct }\end{array}$ & $\begin{array}{c}\text { Dip } \\
\text { Direct }\end{array}$ & Dip & Comments \\
\hline \multicolumn{9}{|l|}{ N1 } \\
\hline & & 1.83 & 1.83 & -0.14 & $\mathrm{~N}-\mathrm{S}$ & W & 77 & top minor subvertical \\
\hline & & 1.83 & 1.83 & -0.47 & $\mathrm{~N}-\mathrm{S}$ & W & 77 & bottom minor subvertical \\
\hline & LBT1 & 1.83 & 1.83 & -0.53 & & & 18 & top large subhorizontal \\
\hline & LBT1 & 1.83 & 1.83 & -0.55 & & & 18 & base large subhorizontal \\
\hline & & 1.83 & 1.83 & -0.55 & NW-SE & SW & 72 & top minor subvertical \\
\hline & & 1.83 & 1.83 & -0.78 & NW-SE & SW & 72 & bottom minor subvertical \\
\hline & & 1.83 & 1.83 & -0.93 & $E-W$ & $\mathrm{~N}$ & & top subhorizontal \\
\hline & & 1.83 & 1.83 & -0.96 & $E-W$ & $\mathrm{~N}$ & & bottom subhorizontal \\
\hline & & 1.83 & 1.83 & -1.59 & $E-W$ & $S$ & 84 & tight fracture \\
\hline & & 1.83 & 1.83 & -2.28 & $E-W$ & $S$ & 84 & tight fracture \\
\hline & & 1.83 & 1.83 & -2.47 & & & & horizontal fracture \\
\hline & & 1.83 & 1.83 & -2.68 & SW-NE & SE & 72 & tight fracture \\
\hline & & 1.83 & 1.83 & -2.91 & SW-NE & SE & 72 & tight fracture \\
\hline & & 1.83 & 1.83 & -3.37 & SW-NE & SE & 34 & open subhorizontal \\
\hline & & 1.83 & 1.83 & -3.42 & SW-NE & SE & 34 & open subhorizontal \\
\hline & & 1.83 & 1.83 & -3.37 & SW-NE & SE & 72 & tight fracture \\
\hline & & 1.83 & 1.83 & -3.60 & SW-NE & SE & 72 & tight fracture \\
\hline & & 1.83 & 1.83 & -3.78 & & & & horizontal fracture \\
\hline & & 1.83 & 1.83 & -4.08 & & & & horizontal fracture \\
\hline & & 1.83 & 1.83 & -4.26 & & & & horizontal fracture \\
\hline & & 1.83 & 1.83 & -4.36 & & & & large horizontal fracture \\
\hline & & 1.83 & 1.83 & -4.69 & & & & subhorizontal? \\
\hline & & 1.83 & 1.83 & -5.10 & & & & horizontal partly open \\
\hline & & 1.83 & 1.83 & -5.28 & & & & horizontal fracture \\
\hline & & 1.83 & 1.83 & -5.30 & NW-SE & SW & 34 & top subhorizontal \\
\hline & & 1.83 & 1.83 & -5.35 & NW-SE & SW & & bottom subhorizontal \\
\hline & & 1.83 & 1.83 & -5.45 & $E-W$ & $\mathrm{NE}$ & 59 & major open fracture \\
\hline & & 1.83 & 1.83 & -5.58 & $E-W$ & $\mathrm{NE}$ & 59 & major open fracture \\
\hline \multicolumn{9}{|l|}{ N2 } \\
\hline & & 1.83 & 1.22 & -0.18 & $E-W$ & $S$ & 63 & minor fracture \\
\hline & & 1.83 & 1.22 & -0.34 & $E-W$ & S & 63 & minor fracture \\
\hline & LBT1 & 1.83 & 1.22 & -0.49 & & & & top large subhorizontal \\
\hline & LBT1 & 1.83 & 1.22 & -0.52 & & & & base large subhorizontal \\
\hline
\end{tabular}




\begin{tabular}{|c|c|c|c|c|c|c|c|c|}
\hline Hole & $\begin{array}{l}\text { Surface } \\
\text { Feature }\end{array}$ & $\begin{array}{c}\text { Model } \\
\text { East }\end{array}$ & $\begin{array}{l}\text { Model } \\
\text { North }\end{array}$ & $\begin{array}{l}\text { Model } \\
\text { Depth }\end{array}$ & $\begin{array}{l}\text { Strike } \\
\text { Direct }\end{array}$ & $\begin{array}{l}\text { Dip } \\
\text { Direct }\end{array}$ & Dip & Comments \\
\hline & & 1.83 & 1.22 & -0.93 & & & & horizontal partly open \\
\hline & LBT17 & 1.83 & 1.22 & -0.93 & $\mathrm{~N}-\mathrm{S}$ & W & & may have alteration halo \\
\hline & LBT17 & 1.83 & 1.22 & -1.35 & $\mathrm{~N}-\mathrm{S}$ & W & & may have alteration halo \\
\hline & & 1.83 & 1.22 & -1.61 & $E-W$ & $\mathrm{~N}$ & 53 & tight fracture \\
\hline & & 1.83 & 1.22 & -1.71 & $E-W$ & $\mathrm{~N}$ & 53 & tight fracture \\
\hline & & 1.83 & 1.22 & -2.95 & $E-W$ & $\mathrm{~N}$ & & top subvertical \\
\hline & & 1.83 & 1.22 & -3.00 & $E-W$ & $\mathrm{~N}$ & & bottom subvertical \\
\hline & & 1.83 & 1.22 & -3.69 & $E-W$ & $\mathrm{~N}$ & & top subhorizontal \\
\hline & & 1.83 & 1.22 & -3.72 & $E-W$ & $\mathrm{~N}$ & & bottom subhorizontal \\
\hline & & 1.83 & 1.22 & -3.94 & $E-W$ & $\mathrm{~N}$ & & top subhorizontal \\
\hline & & 1.83 & 1.22 & -3.97 & $E-W$ & $\mathrm{~N}$ & & bottom subhorizontal \\
\hline & & 1.83 & 1.22 & -4.30 & NW-SE & $\mathrm{NE}$ & & top subhorizontal \\
\hline & & 1.83 & 1.22 & -4.35 & NW-SE & $\mathrm{NE}$ & & bottom subhorizontal \\
\hline & & 1.83 & 1.22 & -5.29 & NW-SE & SW & & top subvertical \\
\hline & & 1.83 & 1.22 & -5.44 & NW-SE & SW & & bottom subvertical \\
\hline \multicolumn{9}{|l|}{ N3 } \\
\hline & & 1.83 & 2.44 & 0.00 & & & & open horizontal fracture \\
\hline & LBT8 & 1.83 & 2.44 & 0.08 & NW-SE & SW & & top subvertical \\
\hline & LBT8 & 1.83 & 2.44 & -0.25 & NW-SE & SW & & bottom subvertical \\
\hline & LBT1 & 1.83 & 2.44 & -0.51 & & & 27 & top large subhorizontal \\
\hline & LBT1 & 1.83 & 2.44 & -0.55 & & & 27 & base large subhorizontal \\
\hline & & 1.83 & 2.44 & -0.99 & SW-NE & $\mathrm{MW}$ & & top subvertical \\
\hline & & 1.83 & 2.44 & -1.19 & SW-NE & $\mathrm{MW}$ & & bottom subvertical \\
\hline & & 1.83 & 2.44 & -1.02 & SW-NE & $M W$ & 72 & $\begin{array}{l}\text { top moderate/major tight } \\
\text { subvertical }\end{array}$ \\
\hline & & 1.83 & 2.44 & -1.24 & SW-NE & MW & 72 & $\begin{array}{l}\text { bottom moderate/major } \\
\text { tight subvertical }\end{array}$ \\
\hline & & 1.83 & 2.44 & -2.11 & & & & horizontal fracture \\
\hline & & 1.83 & 2.44 & -2.79 & $E-W$ & $S$ & 34 & major subhorizontal \\
\hline & & 1.83 & 2.44 & -2.84 & $E-W$ & $S$ & 34 & major subhorizontal \\
\hline & LBT22 & 1.83 & 2.44 & -3.06 & SW-NE & $\mathrm{MW}$ & 71 & major open subvertical \\
\hline & LBT22 & 1.83 & 2.44 & -3.28 & SW-NE & $\mathrm{MW}$ & 71 & major open subvertical \\
\hline & LBT14 & 1.83 & 2.44 & -3.40 & & & 79 & horizontal \\
\hline & LBT14 & 1.83 & 2.44 & -3.81 & & & 79 & horizontal \\
\hline & & 1.83 & 2.44 & -4.17 & & & & horizontal fracture \\
\hline & & 1.83 & 2.44 & -4.27 & & & & horizontal fracture \\
\hline
\end{tabular}




\begin{tabular}{|c|c|c|c|c|c|c|c|c|}
\hline Hole & $\begin{array}{l}\text { Surface } \\
\text { Feature }\end{array}$ & $\begin{array}{c}\text { Model } \\
\text { East }\end{array}$ & $\begin{array}{l}\text { Model } \\
\text { North }\end{array}$ & $\begin{array}{l}\text { Model } \\
\text { Depth }\end{array}$ & $\begin{array}{l}\text { Strike } \\
\text { Direct }\end{array}$ & $\begin{array}{l}\text { Dip } \\
\text { Direct }\end{array}$ & Dip & Comments \\
\hline & & 1.83 & 2.44 & -4.32 & SW-NE & SE & 82 & minor fracture \\
\hline & & 1.83 & 2.44 & -4.34 & & & & horizontal fracture \\
\hline & & 1.83 & 2.44 & -4.52 & & & & horizontal fracture \\
\hline & & 1.83 & 2.44 & -4.75 & & & & horizontal fracture \\
\hline & & 1.83 & 2.44 & -4.88 & SW-NE & SE & & minor \\
\hline & & 1.83 & 2.44 & -5.03 & $E-W$ & & & horizontal fracture \\
\hline & & 1.83 & 2.44 & -5.31 & & & & horizontal fracture \\
\hline \multicolumn{9}{|l|}{ N4 } \\
\hline & LBT1 & 1.52 & 3.05 & -0.43 & & & 18 & top large subhorizontal \\
\hline & LBT1 & 1.52 & 3.05 & -0.46 & & & 18 & base large subhorizontal \\
\hline & & 1.52 & 3.05 & -0.56 & & & 84 & subhorizontal \\
\hline & & 1.52 & 3.05 & -1.23 & & & 84 & subhorizontal \\
\hline & LBT12 & 1.52 & 3.05 & -1.27 & SW-NE & MW & 78 & major subvertical \\
\hline & LBT12 & 1.52 & 3.05 & -1.62 & SW-NE & MW & 78 & major subvertical \\
\hline & & 1.52 & 3.05 & -1.98 & & & & horizontal fracture \\
\hline & LBT13 & 1.52 & 3.05 & -1.98 & $E-W$ & $S ?$ & & $\begin{array}{l}\text { top highly fractured zone- } \\
\text { open major }\end{array}$ \\
\hline & LBT13 & 1.52 & 3.05 & -3.07 & $E-W$ & $S ?$ & & $\begin{array}{l}\text { base highly fractured zone- } \\
\text { open major }\end{array}$ \\
\hline & & 1.52 & 3.05 & -3.43 & SW-NE & MW & 59 & top subvertical \\
\hline & & 1.52 & 3.05 & -3.55 & SW-NE & $\mathrm{MW}$ & 59 & base subvertical \\
\hline & & 1.52 & 3.05 & -3.45 & & & & horizontal fracture \\
\hline & & 1.52 & 3.05 & -3.48 & & & & horizontal fracture \\
\hline & & 1.52 & 3.05 & -3.76 & & & & horizontal fracture \\
\hline & & 1.52 & 3.05 & -3.73 & & & & horizontal fracture \\
\hline & & 1.52 & 3.05 & -3.99 & & & & horizontal fracture \\
\hline & LBT22 & 1.52 & 3.05 & -4.09 & $\mathrm{~N}-\mathrm{S}$ & W & 53 & top major subvertical-open \\
\hline & LBT22 & 1.52 & 3.05 & -4.19 & $\mathrm{~N}-\mathrm{S}$ & W & 53 & base major subvertical-open \\
\hline & & 1.52 & 3.05 & -4.32 & NW-SE & & 69 & top major open fracture? \\
\hline & & 1.52 & 3.05 & -4.52 & NW-SE & & 69 & base major open fracture? \\
\hline & & 1.52 & 3.05 & -4.60 & & & & horizontal fracture \\
\hline & & 1.52 & 3.05 & -4.98 & & & & horizontal fracture \\
\hline & & 1.52 & 3.05 & -5.03 & $E-W$ & $\mathrm{~N}$ & 53 & tight fracture \\
\hline & & 1.52 & 3.05 & -5.13 & $E-W$ & $\mathrm{~N}$ & 53 & tight fracture \\
\hline & & 1.52 & 3.05 & -4.80 & NW-SE & SW & 83 & tight fracture \\
\hline & & 1.52 & 3.05 & -5.38 & NW-SE & SW & 83 & tight fracture \\
\hline
\end{tabular}




\begin{tabular}{|c|c|c|c|c|c|c|c|c|}
\hline Hole & $\begin{array}{l}\text { Surface } \\
\text { Feature }\end{array}$ & $\begin{array}{c}\text { Model } \\
\text { East }\end{array}$ & $\begin{array}{l}\text { Model } \\
\text { North }\end{array}$ & $\begin{array}{l}\text { Model } \\
\text { Depth }\end{array}$ & $\begin{array}{l}\text { Strike } \\
\text { Direct }\end{array}$ & $\begin{array}{l}\text { Dip } \\
\text { Direct }\end{array}$ & Dip & Comments \\
\hline & & 1.52 & 3.05 & -5.41 & SW-NE & SE & 53 & major open fracture \\
\hline & & 1.52 & 3.05 & -5.51 & SW-NE & SE & 53 & major open fracture \\
\hline \multicolumn{9}{|l|}{ N5 } \\
\hline & LBT1 & 0.00 & 1.52 & -0.17 & & & & top large subhorizontal \\
\hline & LBT1 & 0.00 & 1.52 & -0.51 & & & & base large subhorizontal \\
\hline & & 0.00 & 1.52 & -0.29 & $\mathrm{~N}-\mathrm{S}$ & E & 90 & base vertical fracture \\
\hline & LBT2 & 0.00 & 1.52 & -0.24 & $\mathrm{~N}-\mathrm{S}$ & $E$ & 77 & top major? subvertical \\
\hline & LBT2 & 0.00 & 1.52 & -0.57 & $\mathrm{~N}-\mathrm{S}$ & E & 77 & base major? subvertical \\
\hline & & 0.00 & 1.52 & -0.60 & SW-NE & MW & & fracture \\
\hline & & 0.00 & 1.52 & -0.93 & & & & base subvertical \\
\hline & & 0.00 & 1.52 & -1.03 & & & & horizontal fracture \\
\hline & & 0.00 & 1.52 & -1.51 & & & & horizontal fracture \\
\hline & & 0.00 & 1.52 & -1.89 & & & & horizontal fracture \\
\hline & & 0.00 & 1.52 & -2.60 & NW-SE & $N E$ & & $\begin{array}{l}\text { top sharp, open minor } \\
\text { subvertical }\end{array}$ \\
\hline & & 0.00 & 1.52 & -2.76 & NW-SE & $N E$ & & $\begin{array}{l}\text { bottom sharp, open minor } \\
\text { subvertical }\end{array}$ \\
\hline & & 0.00 & 1.52 & -2.86 & & & & horizontal fracture \\
\hline & & 0.00 & 1.52 & -3.16 & & & & horizontal fracture \\
\hline & & 0.00 & 1.52 & -3.47 & & & & horizontal fracture \\
\hline & LBT20 & 0.00 & 1.52 & -3.47 & $E-W$ & $S$ & 78 & $\begin{array}{l}\text { top major subvertical open } \\
\text { fracture }\end{array}$ \\
\hline & LBT20 & 0.00 & 1.52 & -3.82 & $E-W$ & S & 78 & $\begin{array}{l}\text { base major subvertical open } \\
\text { fracture }\end{array}$ \\
\hline & & 0.00 & 1.52 & -3.70 & & & & horizontal fracture \\
\hline & & 0.00 & 1.52 & -3.87 & & & & horizontal fracture \\
\hline & & 0.00 & 1.52 & -4.69 & $\mathrm{~N}-\mathrm{S}$ & W & 81 & top open fracture \\
\hline & & 0.00 & 1.52 & -4.74 & & & & horizontal fracture \\
\hline & & 0.00 & 1.52 & -4.89 & & & & horizontal fracture \\
\hline & & 0.00 & 1.52 & -5.17 & $\mathrm{~N}-\mathrm{S}$ & W & 81 & base open fracture \\
\hline \multicolumn{9}{|l|}{ N6 } \\
\hline & & 1.52 & 0.00 & 0.05 & & & & horizontal fracture \\
\hline & & 1.52 & 0.00 & -0.15 & & & & horizontal fracture \\
\hline & LBT1 & 1.52 & 0.00 & -0.38 & & & 18 & top large subhorizontal \\
\hline & LBT1 & 1.52 & 0.00 & -0.40 & & & 18 & base large subhorizontal \\
\hline
\end{tabular}




\begin{tabular}{|c|c|c|c|c|c|c|c|c|}
\hline Hole & $\begin{array}{l}\text { Surface } \\
\text { Feature }\end{array}$ & $\begin{array}{c}\text { Model } \\
\text { East }\end{array}$ & $\begin{array}{l}\text { Model } \\
\text { North }\end{array}$ & $\begin{array}{l}\text { Model } \\
\text { Depth }\end{array}$ & $\begin{array}{l}\text { Strike } \\
\text { Direct }\end{array}$ & $\begin{array}{l}\text { Dip } \\
\text { Direct }\end{array}$ & Dip & Comments \\
\hline & & 1.52 & 0.00 & -0.66 & $\mathrm{~N}-\mathrm{S}$ & W & 89 & $\begin{array}{l}\text { top subvertical, cannot see } \\
\text { base }\end{array}$ \\
\hline & & 1.52 & 0.00 & -0.73 & & & 89 & horizontal fracture \\
\hline & & 1.52 & 0.00 & -0.81 & & & & horizontal fracture \\
\hline & & 1.52 & 0.00 & -1.22 & & & & horizontal fracture \\
\hline & & 1.52 & 0.00 & -1.57 & & & & horizontal fracture \\
\hline & & 1.52 & 0.00 & -1.67 & & & & horizontal fracture \\
\hline & & 1.52 & 0.00 & 0.00 & $\mathrm{~N}-\mathrm{S}$ & W & & base subvertical \\
\hline & LBT2 & 1.52 & 0.00 & -1.90 & $\mathrm{~N} 45 \mathrm{~W}$ & $\mathrm{NE}$ & 76 & top significant subvertical \\
\hline & LBT2 & 1.52 & 0.00 & -2.21 & N45W & $\mathrm{NE}$ & 76 & base significant subvertical \\
\hline & & 1.52 & 0.00 & -2.10 & & & & horizontal fracture \\
\hline & & 1.52 & 0.00 & -2.66 & & & & horizontal fracture \\
\hline & LBT17 & 1.52 & 0.00 & -2.92 & N30W & SW & 73 & $\begin{array}{l}\text { top major subvertical } \\
\text { (alteration halo?) }\end{array}$ \\
\hline & LBT17 & 1.52 & 0.00 & -3.17 & N30W & SW & 73 & $\begin{array}{l}\text { base major subvertical } \\
\text { (alteration halo?) }\end{array}$ \\
\hline & & 1.52 & 0.00 & -3.65 & & & & horizontal fracture \\
\hline & & 1.52 & 0.00 & -4.49 & $\mathrm{~N}-\mathrm{S}$ & W & 81 & top subvertical \\
\hline & & 1.52 & 0.00 & -4.95 & $\mathrm{~N}-\mathrm{S}$ & W & 81 & base subvertical \\
\hline & & 1.52 & 0.00 & -4.95 & $\mathrm{~N}-\mathrm{S}$ & W & 53 & top subvertical \\
\hline & & 1.52 & 0.00 & -5.05 & $\mathrm{~N}-\mathrm{S}$ & W & 53 & base subvertical \\
\hline & & 1.52 & 0.00 & -5.10 & & & & horizontal fracture \\
\hline & LBT11(?) & 1.52 & 0.00 & -5.25 & N30W & $\mathrm{NE}$ & 82 & major sharp open fracture \\
\hline \multicolumn{9}{|l|}{ N7 } \\
\hline & & 3.05 & 1.52 & -0.24 & & & & horizontal fracture \\
\hline & LBT1 & 3.05 & 1.52 & -0.63 & & & & top large subhorizontal \\
\hline & LBT1 & 3.05 & 1.52 & -0.65 & & & & base large subhorizontal \\
\hline & & 3.05 & 1.52 & -1.06 & & & & horizontal fracture \\
\hline & LBT21 & 3.05 & 1.52 & -1.26 & $\mathrm{~N}-\mathrm{S}$ & W & 76 & top minor subvertical \\
\hline & LBT21 & 3.05 & 1.52 & -1.57 & $\mathrm{~N}-\mathrm{S}$ & W & 76 & base minor subvertical \\
\hline & & 3.05 & 1.52 & -1.49 & & & & horizontal fracture \\
\hline & & 3.05 & 1.52 & -1.92 & & & & horizontal fracture \\
\hline & & 3.05 & 1.52 & -3.27 & & & & horizontal fracture \\
\hline & & 3.05 & 1.52 & -3.88 & & & & horizontal fracture \\
\hline & & 3.05 & 1.52 & -4.41 & $E-W$ & $\mathrm{~N}$ & & top subvertical \\
\hline & & 3.05 & 1.52 & -4.46 & $E-W$ & $\mathrm{~N}$ & & base subvertical \\
\hline
\end{tabular}




\begin{tabular}{|c|c|c|c|c|c|c|c|c|}
\hline Hole & $\begin{array}{l}\text { Surface } \\
\text { Feature }\end{array}$ & $\begin{array}{c}\text { Model } \\
\text { East }\end{array}$ & $\begin{array}{l}\text { Model } \\
\text { North }\end{array}$ & $\begin{array}{l}\text { Model } \\
\text { Depth }\end{array}$ & $\begin{array}{l}\text { Strike } \\
\text { Direct }\end{array}$ & $\begin{array}{l}\text { Dip } \\
\text { Direct }\end{array}$ & Dip & Comments \\
\hline & & 3.05 & 1.52 & -4.72 & & & 90 & $\begin{array}{l}\text { several vertical cooling } \\
\text { joints? }\end{array}$ \\
\hline & & 3.05 & 1.52 & -5.02 & & & & horizontal fracture \\
\hline & & 3.05 & 1.52 & -5.15 & & & & horizontal fracture \\
\hline \multicolumn{9}{|l|}{ E1 } \\
\hline & & 2.44 & 1.22 & -0.09 & & & & horizontal fracture \\
\hline & LBT1 & 2.44 & 1.22 & -0.57 & & & 34 & top large subhorizontal \\
\hline & LBT1 & 2.44 & 1.22 & -0.59 & & & 34 & base large subhorizontal \\
\hline & & 2.44 & 1.22 & -0.92 & $\sim \mathrm{N} 45 \mathrm{~W}$ & NE? & & goes out same side of hole \\
\hline & & 2.44 & 1.22 & -1.03 & & & & horizontal fracture \\
\hline & & 2.44 & 1.22 & -1.10 & $\sim \mathrm{N} 45 \mathrm{~W}$ & $\mathrm{NE} ?$ & & goes out same side of hole \\
\hline & & 2.44 & 1.22 & -1.23 & & & & horizontal fracture \\
\hline & & 2.44 & 1.22 & -1.38 & & & & horizontal fracture \\
\hline & & 2.44 & 1.22 & -1.43 & $\sim \mathrm{N} 45 \mathrm{~W}$ & & & fracture \\
\hline & & 2.44 & 1.22 & -1.79 & & & & horizontal fracture \\
\hline & & 2.44 & 1.22 & -2.78 & & & & base of open fracture \\
\hline & & 2.44 & 1.22 & -3.08 & $M W$ & SW & & significant fracture \\
\hline & & 2.44 & 1.22 & -3.29 & $M W$ & SW & & significant fracture \\
\hline & & 2.44 & 1.22 & -3.34 & $\mathrm{~N}-\mathrm{S}$ & $E$ & & $\begin{array}{l}\text { top open dipping } \\
\text { subhorizontal fracture }\end{array}$ \\
\hline & & 2.44 & 1.22 & -3.36 & $\mathrm{~N}-\mathrm{S}$ & $E$ & & $\begin{array}{l}\text { bottom open dipping } \\
\text { subhorizontal fracture }\end{array}$ \\
\hline & & 2.44 & 1.22 & -3.46 & & & & top rubble continuous \\
\hline & & 2.44 & 1.22 & -3.72 & & & & base rubble zone \\
\hline & & 2.44 & 1.22 & -3.57 & & & & $\begin{array}{l}\text { gaping fracture (cooling } \\
\text { joint?) }\end{array}$ \\
\hline & & 2.44 & 1.22 & -3.72 & & & & leaves same side of hole \\
\hline & & 2.44 & 1.22 & -4.05 & $E-W$ & $S$ & 79 & top subvertical \\
\hline & & 2.44 & 1.22 & -4.25 & $E-W$ & $S$ & 79 & base subvertical \\
\hline & & 2.44 & 1.22 & -4.28 & $E-W$ & $S$ & 78 & top subvertical \\
\hline & & 2.44 & 1.22 & -4.38 & & & & horizontal fracture \\
\hline & & 2.44 & 1.22 & -4.45 & $E-W$ & $S$ & 78 & base subvertical \\
\hline & & 2.44 & 1.22 & -4.53 & & & & horizontal fracture \\
\hline & & 2.44 & 1.22 & -4.61 & & & & top subhorizontal \\
\hline & & 2.44 & 1.22 & -4.66 & & & & bottom subhorizontal \\
\hline & & 2.44 & 1.22 & -4.81 & $E-W$ & S & 69 & top subvertical \\
\hline & & 2.44 & 1.22 & -4.91 & $E-W$ & $\bar{S}$ & 69 & base subvertical \\
\hline
\end{tabular}




\begin{tabular}{|c|c|c|c|c|c|c|c|c|}
\hline Hole & $\begin{array}{l}\text { Surface } \\
\text { Feature }\end{array}$ & $\begin{array}{c}\text { Model } \\
\text { East }\end{array}$ & $\begin{array}{l}\text { Model } \\
\text { North }\end{array}$ & $\begin{array}{l}\text { Model } \\
\text { Depth }\end{array}$ & $\begin{array}{l}\text { Strike } \\
\text { Direct }\end{array}$ & $\begin{array}{l}\text { Dip } \\
\text { Direct }\end{array}$ & Dip & Comments \\
\hline & & 2.44 & 1.22 & -5.17 & $\mathrm{~N}-\mathrm{S}$ & W & 84 & top major open subvertical \\
\hline & & 2.44 & 1.22 & -5.22 & & & & horizontal fracture \\
\hline & & 2.44 & 1.22 & -5.52 & $N-S$ & W & 84 & $\begin{array}{l}\text { bottom major open } \\
\text { subvertical }\end{array}$ \\
\hline \multicolumn{9}{|l|}{ E2 } \\
\hline & & 2.44 & 1.83 & -0.16 & & & & horizontal fracture \\
\hline & LBT1 & 2.44 & 1.83 & -0.59 & & & 34 & top large subhorizontal \\
\hline & LBT1 & 2.44 & 1.83 & -0.62 & & & 34 & base large subhorizontal \\
\hline & & 2.44 & 1.83 & -1.02 & & & & horizontal fracture \\
\hline & & 2.44 & 1.83 & -1.40 & & & & horizontal fracture \\
\hline & & 2.44 & 1.83 & -1.68 & $E-W$ & $S$ & & minor fracture \\
\hline & & 2.44 & 1.83 & -1.86 & $E-W$ & $S$ & & minor fracture \\
\hline & & 2.44 & 1.83 & -1.86 & & & & horizontal fracture \\
\hline & & 2.44 & 1.83 & -1.99 & & & & horizontal fracture \\
\hline & & 2.44 & 1.83 & -2.85 & & & & horizontal fracture \\
\hline & & 2.44 & 1.83 & -3.16 & $\mathrm{~N}-\mathrm{S}$ & & & major open cooling? joint \\
\hline & & 2.44 & 1.83 & -3.46 & $\mathrm{~N}-\mathrm{S}$ & & & major open cooling? joint \\
\hline & & 2.44 & 1.83 & -3.40 & $E-W$ & S & & top minor subhorizontal \\
\hline & & 2.44 & 1.83 & -3.44 & $E-W$ & $S$ & & bottom minor subhorizontal \\
\hline & & 2.44 & 1.83 & -3.56 & $E-W$ & $E$ & & $\begin{array}{l}\text { top minor/moderate } \\
\text { subhorizontal }\end{array}$ \\
\hline & & 2.44 & 1.83 & -3.59 & $E-W$ & E & & $\begin{array}{l}\text { bottom minor/moderate } \\
\text { subhorizontal }\end{array}$ \\
\hline & & 2.44 & 1.83 & -4.02 & & & & horizontal fracture \\
\hline & & 2.44 & 1.83 & -4.07 & & & & horizontal fracture \\
\hline & & 2.44 & 1.83 & -4.15 & $\mathrm{~N}-\mathrm{S}$ & W & & top minor subhorizontal \\
\hline & & 2.44 & 1.83 & -4.20 & $\mathrm{~N}-\mathrm{S}$ & W & & bottom minor subhorizontal \\
\hline & & 2.44 & 1.83 & -4.30 & & & & horizontal fracture \\
\hline & & 2.44 & 1.83 & -4.43 & NW-SE & NE & & $\begin{array}{l}\text { top open moderate sharp } \\
\text { subhorizontal }\end{array}$ \\
\hline & & 2.44 & 1.83 & -4.45 & NW-SE & NE & & $\begin{array}{l}\text { bottom moderate open sharp } \\
\text { subhorizontal }\end{array}$ \\
\hline & & 2.44 & 1.83 & -4.76 & & & & horizontal fracture \\
\hline & & 2.44 & 1.83 & -4.83 & $\mathrm{~N}-\mathrm{S}$ & $E$ & 53 & minor fracture \\
\hline & & 2.44 & 1.83 & -4.88 & $\mathrm{~N}-\mathrm{S}$ & $E$ & 53 & minor fracture \\
\hline & & 2.44 & 1.83 & -4.91 & $N-S$ & & & $\begin{array}{l}\text { major open curving cooling? } \\
\text { joint }\end{array}$ \\
\hline
\end{tabular}




\begin{tabular}{|c|c|c|c|c|c|c|c|c|}
\hline Hole & $\begin{array}{l}\text { Surface } \\
\text { Feature }\end{array}$ & $\begin{array}{l}\text { Model } \\
\text { East }\end{array}$ & $\begin{array}{l}\text { Model } \\
\text { North }\end{array}$ & $\begin{array}{l}\text { Model } \\
\text { Depth }\end{array}$ & $\begin{array}{l}\text { Strike } \\
\text { Direct }\end{array}$ & $\begin{array}{l}\text { Dip } \\
\text { Direct }\end{array}$ & Dip & Comments \\
\hline & & 2.44 & 1.83 & -5.09 & $\mathrm{~N}-\mathrm{S}$ & & & $\begin{array}{l}\text { major open curving cooling? } \\
\text { joint }\end{array}$ \\
\hline & & 2.44 & 1.83 & -5.14 & & & & horizontal fracture \\
\hline & & 2.44 & 1.83 & -5.19 & & & & open joint \\
\hline & & 2.44 & 1.83 & -5.37 & & & & open joint \\
\hline & & 2.44 & 1.83 & -5.26 & $\mathrm{~N}-\mathrm{S}$ & W & 90 & major open vertical joint \\
\hline \multicolumn{9}{|l|}{ E3 } \\
\hline & & 1.83 & 0.61 & 0.03 & $N-S$ & W & & $\begin{array}{l}\text { top subvertical (can't see } \\
\text { base) }\end{array}$ \\
\hline & LBT1 & 1.83 & 0.61 & -0.37 & & & 34 & top large subhorizontal \\
\hline & LBT1 & 1.83 & 0.61 & -0.39 & & & 34 & base large subhorizontal \\
\hline & & 1.83 & 0.61 & -0.79 & & & & horizontal fracture \\
\hline & LBT17 & 1.83 & 0.61 & -0.89 & $\mathrm{~N}-\mathrm{S}$ & W & 79 & major subvertical (halo?) \\
\hline & LBT17 & 1.83 & 0.61 & -1.09 & $\mathrm{~N}-\mathrm{S}$ & W & 79 & major subvertical (halo?) \\
\hline & & 1.83 & 0.61 & -1.09 & NE-SW & & 69 & minor fracture \\
\hline & & 1.83 & 0.61 & -1.19 & NE-SW & & 69 & minor fracture \\
\hline & & 1.83 & 0.61 & -1.24 & & & & horizontal fracture \\
\hline & & 1.83 & 0.61 & -1.80 & & & & horizontal fracture \\
\hline & & 1.83 & 0.61 & -2.77 & & & & horizontal fracture \\
\hline & & 1.83 & 0.61 & -3.07 & & & & horizontal fracture \\
\hline & & 1.83 & 0.61 & -3.68 & & & & horizontal fracture \\
\hline & & 1.83 & 0.61 & -4.34 & & & & horizontal fracture \\
\hline & & 1.83 & 0.61 & 0.00 & $\mathrm{~N}-\mathrm{S}$ & W & & minor fracture \\
\hline & & 1.83 & 0.61 & -4.42 & $\mathrm{~N}-\mathrm{S}$ & W & & minor fracture \\
\hline & & 1.83 & 0.61 & -4.90 & NE-SW & MW & 76 & moderate fracture \\
\hline & & 1.83 & 0.61 & -5.05 & NE-SW & $\mathrm{MW}$ & 76 & moderate fracture \\
\hline & & 1.83 & 0.61 & -5.28 & & & & horizontal fracture \\
\hline \multicolumn{9}{|l|}{ E4 } \\
\hline & & 1.52 & 1.37 & -0.18 & & & & horizontal fracture \\
\hline & LBT1 & 1.52 & 1.37 & -0.38 & & & 34 & top large subhorizontal \\
\hline & LBT1 & 1.52 & 1.37 & -0.41 & & & 34 & base large subhorizontal \\
\hline & & 1.52 & 1.37 & -0.48 & & & & horizontal fracture \\
\hline & & 1.52 & 1.37 & -0.89 & & & & horizontal fracture \\
\hline & & 1.52 & 1.37 & -1.25 & & & & horizontal fracture \\
\hline & & 1.52 & 1.37 & -1.42 & $\mathrm{~N}-\mathrm{S}$ & W & 69 & minor fracture \\
\hline & & 1.52 & 1.37 & -1.53 & $\mathrm{~N}-\mathrm{S}$ & W & 69 & minor fracture \\
\hline
\end{tabular}




\begin{tabular}{|c|c|c|c|c|c|c|c|c|}
\hline Hole & $\begin{array}{l}\text { Surface } \\
\text { Feature }\end{array}$ & $\begin{array}{c}\text { Model } \\
\text { East }\end{array}$ & $\begin{array}{l}\text { Model } \\
\text { North }\end{array}$ & $\begin{array}{l}\text { Model } \\
\text { Depth }\end{array}$ & $\begin{array}{l}\text { Strike } \\
\text { Direct }\end{array}$ & $\begin{array}{l}\text { Dip } \\
\text { Direct }\end{array}$ & Dip & Comments \\
\hline & & 1.52 & 1.37 & -1.58 & $\mathrm{~N}-\mathrm{S}$ & W & & $\begin{array}{l}\text { top open major/moderate } \\
\text { subhorizontal }\end{array}$ \\
\hline & & 1.52 & 1.37 & -1.65 & $\mathrm{~N}-\mathrm{S}$ & W & & $\begin{array}{l}\text { base open major/moderate } \\
\text { subhorizontal }\end{array}$ \\
\hline & & 1.52 & 1.37 & -2.29 & $E-W$ & $S$ & 69 & minor fracture \\
\hline & & 1.52 & 1.37 & -2.39 & $E-W$ & $S$ & 69 & minor fracture \\
\hline & & 1.52 & 1.37 & -2.42 & & & & horizontal fracture \\
\hline & & 1.52 & 1.37 & -3.25 & & & & horizontal fracture \\
\hline & & 1.52 & 1.37 & -3.46 & & & & horizontal fracture \\
\hline & & 1.52 & 1.37 & -3.58 & & & & horizontal fracture \\
\hline & & 1.52 & 1.37 & -3.63 & & & & horizontal fracture \\
\hline & & 1.52 & 1.37 & -3.86 & $E-W$ & $\mathrm{~S}$ & 63 & minor fracture \\
\hline & & 1.52 & 1.37 & -3.94 & $E-W$ & $S$ & 63 & minor fracture \\
\hline & & 1.52 & 1.37 & -3.89 & $\mathrm{~N}-\mathrm{S}$ & W & 63 & minor fracture \\
\hline & & 1.52 & 1.37 & -3.96 & $\mathrm{~N}-\mathrm{S}$ & W & 63 & minor fracture \\
\hline & & 1.52 & 1.37 & -4.12 & & & & horizontal fracture \\
\hline & & 1.52 & 1.37 & -5.13 & & & & horizontal fracture \\
\hline \multicolumn{9}{|l|}{ E5 } \\
\hline & & 1.22 & 0.61 & 0.13 & & & & horizontal fracture \\
\hline & LBT1 & 1.22 & 0.61 & -0.30 & & & & top large subhorizontal \\
\hline & LBT1 & 1.22 & 0.61 & -0.34 & & & & base large subhorizontal \\
\hline & LBT16 & 1.22 & 0.61 & -0.13 & $\mathrm{~N}-\mathrm{S}$ & W & 34 & moderate fracture \\
\hline & LBT16 & 1.22 & 0.61 & -0.15 & $\mathrm{~N}-\mathrm{S}$ & W & 34 & moderate fracture \\
\hline & & 1.22 & 0.61 & -0.20 & $E-W$ & $S$ & 81 & minor subvertical \\
\hline & & 1.22 & 0.61 & -0.43 & $E-W$ & $S$ & 81 & minor subvertical \\
\hline & & 1.22 & 0.61 & -0.74 & & & & horizontal fracture \\
\hline & & 1.22 & 0.61 & -1.14 & & & & horizontal fracture \\
\hline & & 1.22 & 0.61 & -1.55 & & & & horizontal fracture \\
\hline & & 1.22 & 0.61 & -1.57 & & & & horizontal fracture \\
\hline & LBT2 & 1.22 & 0.61 & -2.18 & $\mathrm{~N}-\mathrm{S}$ & $E$ & & top open subvertical \\
\hline & LBT2 & 1.22 & 0.61 & -2.23 & $\mathrm{~N}-\mathrm{S}$ & $E$ & & base open subvertical \\
\hline & & 1.22 & 0.61 & -3.43 & $E-W$ & S & 89 & minor fracture \\
\hline & & 1.22 & 0.61 & 0.00 & $E-W$ & $\mathrm{~S}$ & 89 & minor fracture \\
\hline & & 1.22 & 0.61 & -3.61 & & & & horizontal fracture \\
\hline & & 1.22 & 0.61 & -4.09 & & & & open horizontal fracture \\
\hline & & 1.22 & 0.61 & -4.72 & & & & horizontal fracture \\
\hline
\end{tabular}




\begin{tabular}{|c|c|c|c|c|c|c|c|c|}
\hline Hole & $\begin{array}{l}\text { Surface } \\
\text { Feature }\end{array}$ & $\begin{array}{c}\text { Model } \\
\text { East }\end{array}$ & $\begin{array}{l}\text { Model } \\
\text { North }\end{array}$ & $\begin{array}{l}\text { Model } \\
\text { Depth }\end{array}$ & $\begin{array}{l}\text { Strike } \\
\text { Direct }\end{array}$ & $\begin{array}{l}\text { Dip } \\
\text { Direct }\end{array}$ & Dip & Comments \\
\hline & & 1.22 & 0.61 & -4.77 & & & & horizontal fracture \\
\hline & & 1.22 & 0.61 & -4.88 & $E-W$ & $\mathrm{~S}$ & & minor fracture \\
\hline & & 1.22 & 0.61 & -4.95 & NE-SW & SE & 79 & moderate fracture \\
\hline & & 1.22 & 0.61 & -5.16 & NE-SW & SE & 79 & moderate fracture \\
\hline \multicolumn{9}{|l|}{ E6 } \\
\hline & & & & & & & & horizontal fracture \\
\hline & LBT1 & 1.22 & 1.22 & -0.32 & & & 34 & top large subhorizontal \\
\hline & LBT1 & 1.22 & 1.22 & -0.34 & & & 34 & base large subhorizontal \\
\hline & LBT16 & 1.22 & 1.22 & -0.22 & $\mathrm{~N}-\mathrm{S}$ & W & 63 & moderate open subvertical \\
\hline & LBT16 & 1.22 & 1.22 & -0.29 & $\mathrm{~N}-\mathrm{S}$ & W & 63 & moderate open subvertical \\
\hline & & 1.22 & 1.22 & -1.16 & & & & horizontal fracture \\
\hline & & 1.22 & 1.22 & -1.59 & & & & horizontal fracture \\
\hline & & 1.22 & 1.22 & -2.63 & & & 34 & minor subvertical \\
\hline & & 1.22 & 1.22 & -2.65 & & & 34 & minor subvertical \\
\hline & LBT2 & 1.22 & 1.22 & -3.52 & $\mathrm{~N}-\mathrm{S}$ & E & & highly fractured zone \\
\hline & & 1.22 & 1.22 & -4.05 & $\mathrm{~N}-\mathrm{S}$ & E & & $\begin{array}{l}\text { probably more than one } \\
\text { fracture }\end{array}$ \\
\hline \multicolumn{9}{|l|}{ E7 } \\
\hline & & 1.22 & 1.83 & 0.00 & & & & horizontal fracture \\
\hline & & 1.22 & 1.83 & -0.38 & & & & horizontal fracture \\
\hline & LBT1 & 1.22 & 1.83 & -0.46 & & & 34 & top large subhorizontal \\
\hline & LBT1 & 1.22 & 1.83 & -0.48 & & & 34 & base large subhorizontal \\
\hline & LBT16 & 1.22 & 1.83 & -0.25 & $\mathrm{~N}-\mathrm{S}$ & W & 73 & moderate open fracture \\
\hline & LBT16 & 1.22 & 1.83 & -0.38 & $\mathrm{~N}-\mathrm{S}$ & W & 73 & moderate open fracture \\
\hline & & 1.22 & 1.83 & -0.86 & $\mathrm{~N}-\mathrm{S}$ & W & 69 & minor fracture \\
\hline & & 1.22 & 1.83 & -0.89 & & & 69 & horizontal fracture \\
\hline & & 1.22 & 1.83 & -0.96 & $\mathrm{~N}-\mathrm{S}$ & W & & minor fracture \\
\hline & & 1.22 & 1.83 & -1.09 & & & & $\begin{array}{l}\text { wandering partly open } \\
\text { fracture }\end{array}$ \\
\hline & & 1.22 & 1.83 & -1.55 & & & & $\begin{array}{l}\text { wandering partly open } \\
\text { fracture }\end{array}$ \\
\hline & & 1.22 & 1.83 & -2.16 & $E-W$ & S & & top minor fracture \\
\hline & & 1.22 & 1.83 & -2.39 & $E-W$ & S & & base minor fracture \\
\hline & & 1.22 & 1.83 & -2.62 & & & & horizontal fracture \\
\hline & & 1.22 & 1.83 & -3.17 & & & & horizontal fracture \\
\hline & & 1.22 & 1.83 & -3.61 & & & & horizontal fracture \\
\hline & & 1.22 & 1.83 & -3.71 & & & & horizontal fracture \\
\hline
\end{tabular}




\begin{tabular}{|c|c|c|c|c|c|c|c|c|}
\hline Hole & $\begin{array}{l}\text { Surface } \\
\text { Feature }\end{array}$ & $\begin{array}{c}\text { Model } \\
\text { East }\end{array}$ & $\begin{array}{l}\text { Model } \\
\text { North }\end{array}$ & $\begin{array}{l}\text { Model } \\
\text { Depth }\end{array}$ & $\begin{array}{l}\text { Strike } \\
\text { Direct }\end{array}$ & $\begin{array}{l}\text { Dip } \\
\text { Direct }\end{array}$ & Dip & Comments \\
\hline & & 1.22 & 1.83 & -3.83 & & & & horizontal fracture \\
\hline \multicolumn{9}{|l|}{ E8 } \\
\hline & & 1.22 & 2.44 & -0.01 & & & & horizontal fracture \\
\hline & LBT1 & 1.22 & 2.44 & -0.50 & & & & top large subhorizontal \\
\hline & LBT1 & 1.22 & 2.44 & -0.55 & & & & base large subhorizontal \\
\hline & LBT16 & 1.22 & 2.44 & -0.06 & $\mathrm{~N}-\mathrm{S}$ & W & & $\begin{array}{l}\text { moderate/major open } \\
\text { subvertical }\end{array}$ \\
\hline & LBT16 & 1.22 & 2.44 & -0.11 & $\mathrm{~N}-\mathrm{S}$ & W & & $\begin{array}{l}\text { moderate/major open } \\
\text { subvertical }\end{array}$ \\
\hline & & 1.22 & 2.44 & -0.27 & & & 34 & open subhorizontal fracture \\
\hline & & 1.22 & 2.44 & -0.29 & & & 34 & open subhorizontal fracture \\
\hline & & 1.22 & 2.44 & -1.36 & $E-W$ & $S$ & & minor subvertical \\
\hline & & 1.22 & 2.44 & -1.44 & $E-W$ & $S$ & & minor subvertical \\
\hline & & 1.22 & 2.44 & -1.87 & & & & horizontal fracture \\
\hline & LBT12 & 1.22 & 2.44 & -2.40 & $E-W$ & $S$ & & top major open subvertical \\
\hline & LBT12 & 1.22 & 2.44 & -2.48 & $E-W$ & $S$ & & $\begin{array}{l}\text { bottom major open } \\
\text { subvertical }\end{array}$ \\
\hline & & 1.22 & 2.44 & -2.73 & $E-W$ & $S$ & & $\begin{array}{l}\text { bottom major open } \\
\text { subvertical }\end{array}$ \\
\hline & & 1.22 & 2.44 & -2.65 & $\mathrm{~N}-\mathrm{S}$ & W & 83 & top major open subvertical \\
\hline & & 1.22 & 2.44 & -2.96 & $\mathrm{~N}-\mathrm{S}$ & W & 83 & $\begin{array}{l}\text { bottom major open } \\
\text { subvertical }\end{array}$ \\
\hline & & 1.22 & 2.44 & -2.65 & $E-W$ & $\mathrm{~N}$ & 77 & top major open subvertical \\
\hline & & 1.22 & 2.44 & -2.82 & $E-W$ & $\mathrm{~N}$ & 77 & $\begin{array}{l}\text { bottom major open } \\
\text { subvertical }\end{array}$ \\
\hline & & 1.22 & 2.44 & -3.04 & NE-SW & SE & & major open fracture \\
\hline & & 1.22 & 2.44 & -3.09 & NE-SW & SE & & major open fracture \\
\hline & LBT22 & 1.22 & 2.44 & -3.11 & NE-SW & MW & & major open fracture \\
\hline & LBT22 & 1.22 & 2.44 & -3.19 & NE-SW & $\mathrm{MW}$ & & major open fracture \\
\hline & & 1.22 & 2.44 & -3.44 & $E-W$ & $S$ & 79 & minor fracture \\
\hline & & 1.22 & 2.44 & -3.65 & $E-W$ & $S$ & 79 & minor fracture \\
\hline & & 1.22 & 2.44 & -3.54 & & & & horizontal fracture \\
\hline & & 1.22 & 2.44 & -3.65 & & & & horizontal fracture \\
\hline & & 1.22 & 2.44 & -3.67 & $\mathrm{~N}-\mathrm{S}$ & W & 84 & top major open subvertical \\
\hline & & 1.22 & 2.44 & -4.05 & $\mathrm{~N}-\mathrm{S}$ & W & 84 & $\begin{array}{l}\text { bottom major open } \\
\text { subvertical }\end{array}$ \\
\hline & & 1.22 & 2.44 & -4.26 & NE-SW & SE & 73 & minor fracture \\
\hline & & 1.22 & 2.44 & -4.38 & NE-SW & SE & 73 & minor fracture \\
\hline
\end{tabular}




\begin{tabular}{|c|c|c|c|c|c|c|c|c|}
\hline Hole & $\begin{array}{l}\text { Surface } \\
\text { Feature }\end{array}$ & $\begin{array}{c}\text { Model } \\
\text { East }\end{array}$ & $\begin{array}{l}\text { Model } \\
\text { North }\end{array}$ & $\begin{array}{l}\text { Model } \\
\text { Depth }\end{array}$ & $\begin{array}{l}\text { Strike } \\
\text { Direct }\end{array}$ & $\begin{array}{c}\text { Dip } \\
\text { Direct }\end{array}$ & Dip & Comments \\
\hline & & 1.22 & 2.44 & -4.51 & & & 90 & vertical open joint \\
\hline & & 1.22 & 2.44 & -4.71 & & & 90 & vertical open joint \\
\hline & & 1.22 & 2.44 & -4.76 & & & & subhorizontal fracture \\
\hline & & 1.22 & 2.44 & -4.94 & & & & horizontal fracture \\
\hline & & 1.22 & 2.44 & -5.07 & $\mathrm{~N}-\mathrm{S}$ & W & & tight, minor fracture \\
\hline & & 1.22 & 2.44 & -5.17 & $\mathrm{~N}-\mathrm{S}$ & W & & tight, minor fracture \\
\hline \multicolumn{9}{|l|}{ E9 } \\
\hline & & 0.61 & 1.22 & 0.04 & & & & horizontal fracture \\
\hline & LBT1 & 0.61 & 1.22 & -0.29 & & & 34 & top large subhorizontal \\
\hline & LBT1 & 0.61 & 1.22 & -0.32 & & & 34 & base large subhorizontal \\
\hline & & 0.61 & 1.22 & -0.32 & $\mathrm{~N}-\mathrm{S}$ & W & 63 & tight, minor fracture \\
\hline & & 0.61 & 1.22 & -0.40 & $\mathrm{~N}-\mathrm{S}$ & W & 63 & tight, minor fracture \\
\hline & & 0.61 & 1.22 & -0.73 & & & & horizontal fracture \\
\hline & & 0.61 & 1.22 & -0.95 & & & & horizontal fracture \\
\hline & & 0.61 & 1.22 & -0.95 & SE-NW & SW & 63 & tight, minor fracture \\
\hline & & 0.61 & 1.22 & -1.03 & SE-NW & SW & 63 & tight, minor fracture \\
\hline & & 0.61 & 1.22 & -1.13 & & & & horizontal fracture \\
\hline & LBT16 & 0.61 & 1.22 & -1.13 & $\mathrm{~N}-\mathrm{S}$ & W & & open fracture \\
\hline & & 0.61 & 1.22 & -1.26 & $E-W$ & $\mathrm{~S}$ & 63 & moderate fracture \\
\hline & & 0.61 & 1.22 & -1.34 & $E-W$ & $S$ & 63 & moderate fracture \\
\hline & & 0.61 & 1.22 & -1.44 & $\mathrm{~N}-\mathrm{S}$ & W & 73 & moderate fracture \\
\hline & & 0.61 & 1.22 & -1.56 & $\mathrm{~N}-\mathrm{S}$ & W & 73 & moderate fracture \\
\hline & LBT2 & 0.61 & 1.22 & -1.67 & SE-NW & NE & 76 & major open fracture! \\
\hline & LBT2 & 0.61 & 1.22 & -1.82 & SE-NW & $\mathrm{NE}$ & 76 & major open fracture! \\
\hline & & 0.61 & 1.22 & -3.82 & & & & horizontal fracture \\
\hline & & 0.61 & 1.22 & -4.26 & & & & horizontal fracture \\
\hline & & 0.61 & 1.22 & -5.02 & & & & top subhorizontal \\
\hline & & 0.61 & 1.22 & -5.20 & & & & base subhorizontal \\
\hline \multicolumn{9}{|l|}{ E10 } \\
\hline & & 0.61 & 1.83 & 0.15 & & & & horizontal fracture \\
\hline & LBT1 & 0.61 & 1.83 & -0.36 & & & 34 & top large subhorizontal \\
\hline & LBT1 & 0.61 & 1.83 & -0.38 & & & 34 & base large subhorizontal \\
\hline & & 0.61 & 1.83 & 0.15 & $\mathrm{~N}-\mathrm{S}$ & W & 81 & major open fracture \\
\hline & & 0.61 & 1.83 & -0.10 & $\mathrm{~N}-\mathrm{S}$ & W & 81 & major open fracture \\
\hline & & 0.61 & 1.83 & -1.25 & $\mathrm{~N}-\mathrm{S}$ & W & 63 & major open fracture \\
\hline & & 0.61 & 1.83 & -1.32 & $\mathrm{~N}-\mathrm{S}$ & W & 63 & major open fracture \\
\hline
\end{tabular}




\begin{tabular}{|c|c|c|c|c|c|c|c|c|}
\hline Hole & $\begin{array}{l}\text { Surface } \\
\text { Feature }\end{array}$ & $\begin{array}{c}\text { Model } \\
\text { East }\end{array}$ & $\begin{array}{l}\text { Model } \\
\text { North }\end{array}$ & $\begin{array}{l}\text { Model } \\
\text { Depth }\end{array}$ & $\begin{array}{l}\text { Strike } \\
\text { Direct }\end{array}$ & $\begin{array}{l}\text { Dip } \\
\text { Direct }\end{array}$ & Dip & Comments \\
\hline & LBT16 & 0.61 & 1.83 & -1.27 & $\mathrm{~N}-\mathrm{S}$ & W & 81 & major open fracture \\
\hline & LBT16 & 0.61 & 1.83 & -1.50 & $\mathrm{~N}-\mathrm{S}$ & W & 81 & major open fracture \\
\hline & LBT12 & 0.61 & 1.83 & -2.77 & $E-W$ & $S$ & 78 & major open fracture \\
\hline & LBT12 & 0.61 & 1.83 & -2.95 & $E-W$ & $S$ & 78 & major open fracture \\
\hline & LBT2 & 0.61 & 1.83 & -3.07 & SE-NW & $\mathrm{NE}$ & 79 & major open fracture \\
\hline & LBT2 & 0.61 & 1.83 & -3.28 & SE-NW & NE & 79 & major open fracture \\
\hline & LBT14 & 0.61 & 1.83 & -3.23 & & & & open horizontal fracture \\
\hline & & 0.61 & 1.83 & -3.71 & & & & horizontal fracture \\
\hline & & 0.61 & 1.83 & -3.84 & & & & horizontal fracture \\
\hline & & 0.61 & 1.83 & -5.11 & SW-NE & SE & & minor fracture \\
\hline \multicolumn{9}{|l|}{ EH1 } \\
\hline & LBT21 & 2.84 & 0.30 & -2.74 & & & 90 & $\begin{array}{l}\text { moderate vertical open } \\
\text { fracture }\end{array}$ \\
\hline & & 2.64 & 0.30 & -2.74 & & & 90 & $\begin{array}{l}\text { minor vertical closed } \\
\text { fracture }\end{array}$ \\
\hline & & 2.51 & 0.30 & -2.74 & N60E & SE & 80 & angled partly open fracture \\
\hline & & 2.44 & 0.30 & -2.74 & N60E & SE & 80 & angled partly open fracture \\
\hline & & 2.54 & 0.30 & -2.74 & & & & $\begin{array}{l}\text { closed, healed subhorizontal } \\
\text { fracture }\end{array}$ \\
\hline & & 2.11 & 0.30 & -2.74 & & & & $\begin{array}{l}\text { closed, healed subhorizontal } \\
\text { fracture }\end{array}$ \\
\hline & & 1.98 & 0.30 & -2.74 & & & 90 & minor vertical fracture \\
\hline & & 1.60 & 0.30 & -2.74 & & & 90 & $\begin{array}{l}\text { moderate vertical open } \\
\text { fracture }\end{array}$ \\
\hline & LBT2 & 1.57 & 0.30 & -2.74 & N50W & $N E$ & 80 & $\begin{array}{l}\text { major open angled fracture, } \\
\text { subvertical }\end{array}$ \\
\hline & LBT2 & 1.52 & 0.30 & -2.74 & N50W & $N E$ & 80 & $\begin{array}{l}\text { major open angled fracture, } \\
\text { subvertical }\end{array}$ \\
\hline & LBT17(?) & 1.50 & 0.30 & -2.74 & & & 90 & minor vertical fracture \\
\hline & & 1.45 & 0.30 & -2.74 & $\mathrm{~N} 30 \mathrm{E}$ & & 90 & $\begin{array}{l}\text { major open fracture, } \\
\text { vertical }\end{array}$ \\
\hline & & 1.42 & 0.30 & -2.74 & $\mathrm{~N} 30 \mathrm{E}$ & & 90 & $\begin{array}{l}\text { major open fracture, } \\
\text { vertical }\end{array}$ \\
\hline & & 0.74 & 0.30 & -2.74 & NE-SW & & & $\begin{array}{l}\text { minor subvertical closed } \\
\text { fracture }\end{array}$ \\
\hline \multicolumn{9}{|l|}{$\mathrm{EH} 2$} \\
\hline & LBT21 & 2.79 & 0.91 & -2.74 & & & 90 & significant vertical fracture \\
\hline & & 2.74 & 0.91 & -2.74 & & & 90 & $\begin{array}{l}\text { significant vertical } \\
\text { fracture, open }\end{array}$ \\
\hline
\end{tabular}




\begin{tabular}{|c|c|c|c|c|c|c|c|c|}
\hline Hole & $\begin{array}{l}\text { Surface } \\
\text { Feature }\end{array}$ & $\begin{array}{c}\text { Model } \\
\text { East }\end{array}$ & $\begin{array}{l}\text { Model } \\
\text { North }\end{array}$ & $\begin{array}{l}\text { Model } \\
\text { Depth }\end{array}$ & $\begin{array}{l}\text { Strike } \\
\text { Direct }\end{array}$ & $\begin{array}{l}\text { Dip } \\
\text { Direct }\end{array}$ & Dip & Comments \\
\hline & & 1.89 & 0.91 & -2.74 & & & & $\begin{array}{l}\text { minor subvertical closed } \\
\text { fracture }\end{array}$ \\
\hline & & 1.57 & 0.91 & -2.74 & NE-SW & & & subvertical open fracture \\
\hline & LBT17(?) & 1.57 & 0.91 & -2.74 & N30W & SW & 70 & moderate open fracture \\
\hline & LBT17(?) & 1.55 & 0.91 & -2.74 & N30W & SW & 70 & moderate open fracture \\
\hline & & 1.14 & 0.91 & -2.74 & NW-SE & SW & & $\begin{array}{l}\text { moderate partly open } \\
\text { subvertical fracture }\end{array}$ \\
\hline & & 0.74 & 0.91 & -2.74 & & & & $\begin{array}{l}\text { minor random irregular } \\
\text { closed fractures }\end{array}$ \\
\hline & & 0.30 & 0.91 & -2.74 & & & & $\begin{array}{l}\text { minor random irregular } \\
\text { closed fractures }\end{array}$ \\
\hline \multicolumn{9}{|l|}{ EH3 } \\
\hline & & 2.79 & 1.52 & -2.74 & & & 90 & $\begin{array}{l}\text { minor vertical closed } \\
\text { fracture }\end{array}$ \\
\hline & & 2.67 & 1.52 & -2.74 & & & 90 & $\begin{array}{l}\text { minor vertical closed } \\
\text { fracture, partly open }\end{array}$ \\
\hline & & 2.44 & 1.52 & -2.74 & & & 90 & $\begin{array}{l}\text { minor vertical closed } \\
\text { fracture }\end{array}$ \\
\hline & & 1.85 & 1.52 & -2.74 & & & 90 & $\begin{array}{l}\text { minor vertical closed } \\
\text { fracture }\end{array}$ \\
\hline & & 1.40 & 1.52 & -2.74 & & & 90 & $\begin{array}{l}\text { minor vertical closed } \\
\text { fracture }\end{array}$ \\
\hline & & 1.37 & 1.52 & -2.74 & N10E & & 90 & $\begin{array}{l}\text { major open vertical } \\
\text { fracture }\end{array}$ \\
\hline & & 1.19 & 1.52 & -2.74 & & & 90 & $\begin{array}{l}\text { minor partly open vertical } \\
\text { fracture }\end{array}$ \\
\hline & & 0.97 & 1.52 & -2.74 & & & 90 & $\begin{array}{l}\text { minor partly open vertical } \\
\text { fracture }\end{array}$ \\
\hline & LBT2(?) & 0.66 & 1.52 & -2.74 & N40W & $\mathrm{NE}$ & 70 & $\begin{array}{l}\text { angled vertical tight } \\
\text { fracture }\end{array}$ \\
\hline & LBT2(?) & 0.61 & 1.52 & -2.74 & N40W & $\mathrm{NE}$ & 70 & $\begin{array}{l}\text { angled vertical tight } \\
\text { fracture }\end{array}$ \\
\hline \multicolumn{9}{|l|}{$\mathrm{EH} 4$} \\
\hline & & 2.79 & 2.13 & -2.74 & & & 90 & $\begin{array}{l}\text { minor vertical partly open } \\
\text { fracture }\end{array}$ \\
\hline & & 2.77 & 2.13 & -2.74 & & & 90 & $\begin{array}{l}\text { minor vertical partly open } \\
\text { fracture }\end{array}$ \\
\hline & LBT6 & 2.64 & 2.13 & -2.74 & N10W & & 90 & $\begin{array}{l}\text { major(?) open vertical } \\
\text { fracture }\end{array}$ \\
\hline
\end{tabular}




\begin{tabular}{|c|c|c|c|c|c|c|c|c|}
\hline Hole & $\begin{array}{l}\text { Surface } \\
\text { Feature }\end{array}$ & $\begin{array}{c}\text { Model } \\
\text { East }\end{array}$ & $\begin{array}{l}\text { Model } \\
\text { North }\end{array}$ & $\begin{array}{l}\text { Model } \\
\text { Depth }\end{array}$ & $\begin{array}{l}\text { Strike } \\
\text { Direct }\end{array}$ & $\begin{array}{l}\text { Dip } \\
\text { Direct }\end{array}$ & Dip & Comments \\
\hline & & 2.57 & 2.13 & -2.74 & NW-SE & & 90 & $\begin{array}{l}\text { minor-moderate angled } \\
\text { partly open vertical } \\
\text { fracture }\end{array}$ \\
\hline & & 2.54 & 2.13 & -2.74 & NW-SE & & 90 & $\begin{array}{l}\text { minor-moderate angled } \\
\text { partly open vertical } \\
\text { fracture }\end{array}$ \\
\hline & & 2.57 & 2.13 & -2.74 & NE-SW & & & minor subvertical fracture \\
\hline & & 2.51 & 2.13 & -2.74 & NE-SW & & & minor subvertical fracture \\
\hline & & 2.06 & 2.13 & -2.74 & & & 90 & $\begin{array}{l}\text { major open vertical } \\
\text { fracture }\end{array}$ \\
\hline & LBT12(?) & 2.03 & 2.13 & -2.74 & & & 90 & $\begin{array}{l}\text { major open vertical } \\
\text { fracture, less significant } \\
\text { then above }\end{array}$ \\
\hline & & 1.78 & 2.13 & -2.74 & N20E & $M W$ & 90 & $\begin{array}{l}\text { major vertical open } \\
\text { fracture }\end{array}$ \\
\hline & & 1.65 & 2.13 & -2.74 & & & 90 & minor vertical fracture \\
\hline & & 1.50 & 2.13 & -2.74 & & & 90 & $\begin{array}{l}\text { minor fine fracture, } \\
\text { vertical barely open }\end{array}$ \\
\hline & & 1.45 & 2.13 & -2.74 & & & 90 & $\begin{array}{l}\text { minor fine fracture, } \\
\text { vertical barely open }\end{array}$ \\
\hline & & 1.40 & 2.13 & -2.74 & & & 90 & $\begin{array}{l}\text { minor fine fracture, } \\
\text { vertical barely open }\end{array}$ \\
\hline & & 1.32 & 2.13 & -2.74 & & & 90 & $\begin{array}{l}\text { open vertical fracture, } \\
\text { more significant then above }\end{array}$ \\
\hline & LBT17(?) & 1.09 & 2.13 & -2.74 & $\mathrm{~N} 60 \mathrm{E}$ & SE & 50 & major angled open fracture \\
\hline & LBT17(?) & 1.04 & 2.13 & -2.74 & $\mathrm{~N} 60 \mathrm{E}$ & SE & 50 & major angled open fracture \\
\hline & & 0.99 & 2.13 & -2.74 & N60E & SE & 45 & major angled open fracture \\
\hline & & 0.91 & 2.13 & -2.74 & N60E & SE & 45 & major angled open fracture \\
\hline & LBT23 & 0.81 & 2.13 & -2.74 & $\mathrm{~N} 20 \mathrm{E}$ & $\mathrm{MW}$ & 45 & major angled open fracture \\
\hline & LBT23 & 0.76 & 2.13 & -2.74 & $\mathrm{~N} 20 \mathrm{E}$ & $\mathrm{MW}$ & 45 & major angled open fracture \\
\hline \multicolumn{9}{|l|}{ EH5 } \\
\hline & & 2.84 & 2.74 & -2.74 & & & 90 & $\begin{array}{l}\text { major open vertical } \\
\text { fracture }\end{array}$ \\
\hline & & 2.82 & 2.74 & -2.74 & $N-S$ & E & 20 & $\begin{array}{l}\text { minor tight closed } \\
\text { subhorizontal healed } \\
\text { fracture }\end{array}$ \\
\hline & & 2.69 & 2.74 & -2.74 & $\mathrm{~N}-\mathrm{S}$ & $E$ & 20 & $\begin{array}{l}\text { minor tight closed } \\
\text { subhorizontal healed } \\
\text { fracture }\end{array}$ \\
\hline & & 2.69 & 2.74 & -2.74 & & & 90 & $\begin{array}{l}\text { minor vertical partly open } \\
\text { fracture }\end{array}$ \\
\hline
\end{tabular}




\begin{tabular}{|c|c|c|c|c|c|c|c|c|}
\hline Hole & $\begin{array}{l}\text { Surface } \\
\text { Feature }\end{array}$ & $\begin{array}{c}\text { Model } \\
\text { East }\end{array}$ & $\begin{array}{l}\text { Model } \\
\text { North }\end{array}$ & $\begin{array}{l}\text { Model } \\
\text { Depth }\end{array}$ & $\begin{array}{l}\text { Strike } \\
\text { Direct }\end{array}$ & $\begin{array}{l}\text { Dip } \\
\text { Direct }\end{array}$ & Dip & Comments \\
\hline & & 2.64 & 2.74 & -2.74 & N30E & SE & 80 & $\begin{array}{l}\text { moderate angled open } \\
\text { fracture }\end{array}$ \\
\hline & & 2.62 & 2.74 & -2.74 & $\mathrm{~N} 30 \mathrm{E}$ & SE & 80 & $\begin{array}{l}\text { moderate angled open } \\
\text { fracture }\end{array}$ \\
\hline & & 2.29 & 2.74 & -2.74 & $N-S$ & W & & $\begin{array}{l}\text { minor tight subvertical } \\
\text { fracture }\end{array}$ \\
\hline & & 2.26 & 2.74 & -2.74 & $N-S$ & W & & $\begin{array}{l}\text { minor tight subvertical } \\
\text { fracture }\end{array}$ \\
\hline & LBT6 & 2.13 & 2.74 & -2.74 & N20W & & 90 & $\begin{array}{l}\text { major open vertical } \\
\text { fracture zone }\end{array}$ \\
\hline & & 1.88 & 2.74 & -2.74 & $\mathrm{~N}-\mathrm{S}$ & E & 20 & $\begin{array}{l}\text { moderate subhorizontal } \\
\text { fracture, with assoc. other } \\
\text { minors }\end{array}$ \\
\hline & & 1.73 & 2.74 & -2.74 & $\mathrm{~N}-\mathrm{S}$ & E & 20 & $\begin{array}{l}\text { moderate subhorizontal } \\
\text { fracture, with assoc. other } \\
\text { minors }\end{array}$ \\
\hline & LBT13(?) & 1.60 & 2.74 & -2.74 & & & 90 & minor vertical fracture \\
\hline & LBT23 & 1.49 & 2.74 & -2.74 & N30E & & 80 & $\begin{array}{l}\text { moderate open vertical } \\
\text { fracture }\end{array}$ \\
\hline & LBT12(?) & 1.22 & 2.74 & -2.74 & & & 90 & $\begin{array}{l}\text { major open vertical sharp } \\
\text { fracture }\end{array}$ \\
\hline & & 0.99 & 2.74 & -2.74 & $N-S$ & E & 20 & $\begin{array}{l}\text { moderate subhorizontal } \\
\text { closed fracture }\end{array}$ \\
\hline & & 0.89 & 2.74 & -2.74 & $N-S$ & $E$ & 20 & $\begin{array}{l}\text { moderate subhorizontal } \\
\text { closed fracture }\end{array}$ \\
\hline & & 0.91 & 2.74 & -2.74 & N80W & & 80 & $\begin{array}{l}\text { angled moderate(?) partly } \\
\text { open fractures }\end{array}$ \\
\hline & & 0.71 & 2.74 & -2.74 & N80W & & 80 & $\begin{array}{l}\text { angled moderate(?) partly } \\
\text { open fractures }\end{array}$ \\
\hline & & 0.61 & 2.74 & -2.74 & & & & $\begin{array}{l}\text { minor subvertical closed } \\
\text { fracture, healed }\end{array}$ \\
\hline & & 0.53 & 2.74 & -2.74 & & & & $\begin{array}{l}\text { minor subvertical closed } \\
\text { fracture }\end{array}$ \\
\hline \multicolumn{9}{|l|}{ E03 } \\
\hline & LBT7 & 2.93 & 2.74 & -3.96 & & & 90 & vertical open fracture \\
\hline & & 2.54 & 2.74 & -3.96 & $\mathrm{~N}-\mathrm{S}$ & $E$ & 30 & moderate closed fracture \\
\hline & & 2.49 & 2.74 & -3.96 & $\mathrm{~N}-\mathrm{S}$ & $E$ & 30 & moderate closed fracture \\
\hline & & 2.46 & 2.74 & -3.96 & N60W & $M W$ & & $\begin{array}{l}\text { angled moderate fracture, } \\
\text { open, subvertical }\end{array}$ \\
\hline & & 2.44 & 2.74 & -3.96 & N60W & $\mathrm{MW}$ & & $\begin{array}{l}\text { angled moderate fracture, } \\
\text { open, subvertical }\end{array}$ \\
\hline
\end{tabular}




\begin{tabular}{|c|c|c|c|c|c|c|c|c|}
\hline Hole & $\begin{array}{l}\text { Surface } \\
\text { Feature }\end{array}$ & $\begin{array}{c}\text { Model } \\
\text { East }\end{array}$ & $\begin{array}{l}\text { Model } \\
\text { North }\end{array}$ & $\begin{array}{l}\text { Model } \\
\text { Depth }\end{array}$ & $\begin{array}{l}\text { Strike } \\
\text { Direct }\end{array}$ & $\begin{array}{l}\text { Dip } \\
\text { Direct }\end{array}$ & Dip & Comments \\
\hline & & 2.36 & 2.74 & -3.96 & $E-W$ & & 90 & $\begin{array}{l}\text { moderate partly open } \\
\text { fracture }\end{array}$ \\
\hline & & 2.31 & 2.74 & -3.96 & $E-W$ & & 90 & $\begin{array}{l}\text { moderate partly open } \\
\text { fracture }\end{array}$ \\
\hline & & 2.24 & 2.74 & -3.96 & & & 90 & minor vertical fracture \\
\hline & & 2.04 & 2.74 & -3.96 & $\mathrm{~N} 45 \mathrm{E}$ & & 90 & angled minor closed fracture \\
\hline & & 1.98 & 2.74 & -3.96 & $\mathrm{~N} 45 \mathrm{E}$ & & 90 & angled minor closed fracture \\
\hline & LBT6 & 1.78 & 2.74 & -3.96 & N70W & $\mathrm{NE}$ & 80 & major angled fracture \\
\hline & LBT6 & 1.65 & 2.74 & -3.96 & N70W & $\mathrm{NE}$ & 80 & major angled fracture \\
\hline & & 1.47 & 2.74 & -3.96 & $\mathrm{~N} 30 \mathrm{E}$ & & 90 & $\begin{array}{l}\text { major angled locally open } \\
\text { fracture }\end{array}$ \\
\hline & & 1.42 & 2.74 & -3.96 & $\mathrm{~N} 30 \mathrm{E}$ & & 90 & $\begin{array}{l}\text { major angled locally open } \\
\text { fracture }\end{array}$ \\
\hline & & 1.42 & 2.74 & -3.96 & & & & $\begin{array}{l}2 \text { open moderate subvertical } \\
\text { fractures }\end{array}$ \\
\hline & & 1.40 & 2.74 & -3.96 & & & & $\begin{array}{l}2 \text { open moderate subvertical } \\
\text { fractures }\end{array}$ \\
\hline & & 1.30 & 2.74 & -3.96 & & & 90 & $\begin{array}{l}\text { major large open vertical } \\
\text { fractures }\end{array}$ \\
\hline & & 0.99 & 2.74 & -3.96 & & & & $\begin{array}{l}\text { minor subvertical tight } \\
\text { fractures }\end{array}$ \\
\hline & & 0.97 & 2.74 & -3.96 & & & & $\begin{array}{l}\text { minor subvertical tight } \\
\text { fractures }\end{array}$ \\
\hline & & 0.66 & 2.74 & -3.96 & $\sim \mathrm{N}-\mathrm{S}$ & $E$ & 10 & $\begin{array}{l}\text { major open subhorizontal } \\
\text { fracture, complex system }\end{array}$ \\
\hline & & 0.38 & 2.74 & -3.96 & $\sim \mathrm{N}-\mathrm{S}$ & E & 10 & $\begin{array}{l}\text { major open subhorizontal } \\
\text { fracture, complex system }\end{array}$ \\
\hline \multicolumn{9}{|l|}{$\mathrm{NH} 1$} \\
\hline & LBT22 & 2.13 & 2.72 & -2.44 & NE-SW & $\mathrm{MW}$ & 80 & major open angled fracture \\
\hline & LBT22 & 2.13 & 2.06 & -2.44 & NE-SW & MW & 80 & major open angled fracture \\
\hline & & 2.13 & 1.80 & -2.44 & $E-W$ & & 90 & $\begin{array}{l}\text { minor tight vertical } \\
\text { fracture }\end{array}$ \\
\hline & & 2.13 & 1.09 & -2.44 & $E-W$ & & 90 & $\begin{array}{l}\text { moderate-major vertical } \\
\text { partly open fracture }\end{array}$ \\
\hline & & 2.13 & 0.66 & -2.44 & N30W & SW & 60 & $\begin{array}{l}\text { minor-moderate angled tight } \\
\text { fracture }\end{array}$ \\
\hline & & 2.13 & 0.28 & -2.44 & N30W & SW & 60 & $\begin{array}{l}\text { minor-moderate angled tight } \\
\text { fracture }\end{array}$ \\
\hline
\end{tabular}




\begin{tabular}{|c|c|c|c|c|c|c|c|c|}
\hline Hole & $\begin{array}{l}\text { Surface } \\
\text { Feature }\end{array}$ & $\begin{array}{c}\text { Model } \\
\text { East }\end{array}$ & $\begin{array}{l}\text { Model } \\
\text { North }\end{array}$ & $\begin{array}{l}\text { Model } \\
\text { Depth }\end{array}$ & $\begin{array}{l}\text { Strike } \\
\text { Direct }\end{array}$ & $\begin{array}{l}\text { Dip } \\
\text { Direct }\end{array}$ & Dip & Comments \\
\hline \multicolumn{9}{|l|}{ NM1 } \\
\hline & & 2.74 & 2.64 & -3.81 & & & & $\begin{array}{l}\text { series of tight minor } \\
\text { vertical to subvertical } \\
\text { fractures }\end{array}$ \\
\hline & & 2.74 & 2.49 & -3.81 & & & & $\begin{array}{l}\text { series of tight minor } \\
\text { vertical to subvertical } \\
\text { fractures }\end{array}$ \\
\hline & LBT8 & 2.74 & 1.80 & -3.81 & $\mathrm{~N} 80 \mathrm{E}$ & $S$ & 70 & major subvertical fracture \\
\hline & LBT8 & 2.74 & 1.73 & -3.81 & $\mathrm{~N} 80 \mathrm{E}$ & $S$ & 70 & major subvertical fracture \\
\hline & & 2.74 & 1.73 & -3.81 & & & & $\begin{array}{l}\text { series of minor subvertical } \\
\text { fractures }\end{array}$ \\
\hline & & 2.74 & 1.63 & -3.81 & & & & $\begin{array}{l}\text { series of minor subvertical } \\
\text { fractures }\end{array}$ \\
\hline & LBT3 & 2.74 & 1.50 & -3.81 & & & 90 & $\begin{array}{l}\text { minor-moderate partly open } \\
\text { vertical fracture }\end{array}$ \\
\hline & & 2.74 & 1.09 & -3.81 & $\mathrm{~N} 45 \mathrm{~W}$ & SW & 80 & $\begin{array}{l}\text { angled minor subvertical } \\
\text { fracture }\end{array}$ \\
\hline & & 2.74 & 0.99 & -3.81 & N45W & SW & 80 & $\begin{array}{l}\text { angled minor subvertical } \\
\text { fracture }\end{array}$ \\
\hline \multicolumn{9}{|l|}{ NM2 } \\
\hline & & 0.91 & 3.02 & -2.44 & $E-W$ & & & $\begin{array}{l}\text { minor-moderate tight } \\
\text { fracture }\end{array}$ \\
\hline & & 0.91 & 2.72 & -2.44 & $E-W$ & & & $\begin{array}{l}\text { minor-moderate tight } \\
\text { fracture }\end{array}$ \\
\hline & & 0.91 & 2.29 & -2.44 & $E-W$ & & 90 & $\begin{array}{l}\text { major open vertical } \\
\text { fracture }\end{array}$ \\
\hline & & 0.91 & 1.93 & -2.44 & $E-W$ & $N ?$ & & $\begin{array}{l}\text { tough to see, but looks like a } \\
\text { minor fracture }\end{array}$ \\
\hline & & 0.91 & 1.83 & -2.44 & & & & $\begin{array}{l}\text { minor tight subvertical } \\
\text { fracture }\end{array}$ \\
\hline & & 0.91 & 1.73 & -2.44 & & & & $\begin{array}{l}\text { moderate open subvertical } \\
\text { fracture }\end{array}$ \\
\hline & LBT2 & 0.91 & 1.12 & -2.44 & $\mathrm{~N} 45 \mathrm{~W}$ & SW & 70 & major angled open fracture \\
\hline & LBT2 & 0.91 & 0.99 & -2.44 & $\mathrm{~N} 45 \mathrm{~W}$ & SW & 70 & major angled open fracture \\
\hline & LBT3 & 0.91 & 0.97 & -2.44 & $E-W$ & & 90 & $\begin{array}{l}\text { moderate-major vertical } \\
\text { open fracture }\end{array}$ \\
\hline & & 0.91 & 0.61 & -2.44 & $E-W$ & & & minor tight fracture \\
\hline & & 0.91 & 0.28 & -2.44 & $E-W$ & & & minor tight fracture \\
\hline \multicolumn{9}{|l|}{ NM3 } \\
\hline & LBT24 & 0.30 & 2.39 & -0.91 & NE-SW & $\mathrm{MW}$ & & $\begin{array}{l}\text { moderate angled tight } \\
\text { fracture }\end{array}$ \\
\hline
\end{tabular}




\begin{tabular}{|c|c|c|c|c|c|c|c|c|}
\hline Hole & $\begin{array}{l}\text { Surface } \\
\text { Feature }\end{array}$ & $\begin{array}{c}\text { Model } \\
\text { East }\end{array}$ & $\begin{array}{l}\text { Model } \\
\text { North }\end{array}$ & $\begin{array}{l}\text { Model } \\
\text { Depth }\end{array}$ & $\begin{array}{l}\text { Strike } \\
\text { Direct }\end{array}$ & $\begin{array}{l}\text { Dip } \\
\text { Direct }\end{array}$ & Dip & Comments \\
\hline & LBT24 & 0.30 & 2.21 & -0.91 & NE-SW & $\mathrm{MW}$ & & $\begin{array}{l}\text { moderate angled tight } \\
\text { fracture }\end{array}$ \\
\hline & LBT2 & 0.30 & 1.24 & -0.91 & NW-SE & NE & & major open angled fracture \\
\hline & & 0.30 & 1.12 & -0.91 & NW-SE & NE & & major open angled fracture \\
\hline & LBT3 & 0.30 & 1.07 & -0.91 & & & 90 & $\begin{array}{l}\text { moderate open(?) vertical } \\
\text { fracture }\end{array}$ \\
\hline & & 0.30 & 0.48 & -0.91 & $E-W$ & & 90 & $\begin{array}{l}\text { moderate-major vertical } \\
\text { open wet fracture }\end{array}$ \\
\hline \multicolumn{9}{|l|}{ NN1 } \\
\hline & & 2.13 & 2.87 & -0.91 & NW-SE & NE & & $\begin{array}{l}\text { moderate-major angled } \\
\text { partly open fracture }\end{array}$ \\
\hline & & 2.13 & 2.79 & -0.91 & NW-SE & NE & & $\begin{array}{l}\text { moderate-major angled } \\
\text { partly open fracture }\end{array}$ \\
\hline & LBT8 & 2.13 & 2.24 & -0.91 & NW-SE & $S ?$ & 90 & $\begin{array}{l}\text { minor tight nearly vertical } \\
\text { fracture }\end{array}$ \\
\hline & & 2.13 & 2.18 & -0.91 & & & 90 & $\begin{array}{l}\text { moderate vertical open wet } \\
\text { fracture }\end{array}$ \\
\hline & & 2.13 & 2.11 & -0.91 & & & 90 & $\begin{array}{l}\text { minor vertical open wet } \\
\text { fracture }\end{array}$ \\
\hline & & 2.13 & 1.83 & -0.91 & & & 90 & $\begin{array}{l}\text { minor nearly vertical closed } \\
\text { fracture }\end{array}$ \\
\hline & & 2.13 & 1.30 & -0.91 & NW-SE & SW & & $\begin{array}{l}\text { moderate-major angled } \\
\text { fracture partly open }\end{array}$ \\
\hline & & 2.13 & 1.17 & -0.91 & NW-SE & SW & & $\begin{array}{l}\text { moderate-major angled } \\
\text { fracture partly open }\end{array}$ \\
\hline & & 2.13 & 1.02 & -0.91 & & & 90 & $\begin{array}{l}\text { moderate open wet vertical } \\
\text { fracture }\end{array}$ \\
\hline & & 2.13 & 0.56 & -0.91 & & & 90 & $\begin{array}{l}\text { vertical partly open } \\
\text { fracture }\end{array}$ \\
\hline & & 2.13 & 0.33 & -0.91 & & & 90 & $\begin{array}{l}\text { vertical open moderate- } \\
\text { major fracture }\end{array}$ \\
\hline \multicolumn{9}{|l|}{ NN2 } \\
\hline & LBT6 & 2.13 & 2.91 & -1.98 & & & & $\begin{array}{l}\text { major?, healed? vertical } \\
\text { fracture }\end{array}$ \\
\hline & & 2.13 & 2.72 & -1.98 & & & & $\begin{array}{l}\text { minor tight vertical } \\
\text { fracture }\end{array}$ \\
\hline & & 2.13 & 2.64 & -1.98 & N45E & & & $\begin{array}{l}\text { angled minor tight } \\
\text { subvertical fracture }\end{array}$ \\
\hline & & 2.13 & 2.62 & -1.98 & N45E & & & $\begin{array}{l}\text { angled minor tight } \\
\text { subvertical fracture }\end{array}$ \\
\hline
\end{tabular}




\begin{tabular}{|c|c|c|c|c|c|c|c|c|}
\hline Hole & $\begin{array}{l}\text { Surface } \\
\text { Feature }\end{array}$ & $\begin{array}{l}\text { Model } \\
\text { East }\end{array}$ & $\begin{array}{l}\text { Model } \\
\text { North }\end{array}$ & $\begin{array}{l}\text { Model } \\
\text { Depth }\end{array}$ & $\begin{array}{l}\text { Strike } \\
\text { Direct }\end{array}$ & $\begin{array}{l}\text { Dip } \\
\text { Direct }\end{array}$ & Dip & Comments \\
\hline & & 2.13 & 2.24 & -1.98 & N45E & & & $\begin{array}{l}\text { moderate tight angled } \\
\text { subvertical fracture }\end{array}$ \\
\hline & & 2.13 & 2.18 & -1.98 & N45E & & & $\begin{array}{l}\text { moderate tight angled } \\
\text { subvertical fracture }\end{array}$ \\
\hline & & 2.13 & 1.93 & -1.98 & $E-W$ & & 90 & $\begin{array}{l}\text { minor/moderate tight } \\
\text { fracture }\end{array}$ \\
\hline & & 2.13 & 1.35 & -1.98 & $E-W$ & & 90 & $\begin{array}{l}\text { minor } / \text { moderate tight } \\
\text { fracture }\end{array}$ \\
\hline & & 2.13 & 1.23 & -1.98 & N30W & SW & 70 & $\begin{array}{l}\text { minor-moderate partly open } \\
\text { angled fracture }\end{array}$ \\
\hline & & 2.13 & 1.04 & -1.98 & N30W & SW & 70 & $\begin{array}{l}\text { minor-moderate partly open } \\
\text { angled fracture }\end{array}$ \\
\hline & & 2.13 & 1.17 & -1.98 & $\sim \mathrm{N}-\mathrm{S}$ & W & & $\begin{array}{l}\text { major-moderate fracture } \\
\text { (leaves same side of hole) }\end{array}$ \\
\hline & & 2.13 & 1.07 & -1.98 & $\sim \mathrm{N}-\mathrm{S}$ & W & & $\begin{array}{l}\text { major-moderate fracture } \\
\text { (leaves same side of hole) }\end{array}$ \\
\hline & LBT3 & 2.13 & 1.09 & -1.98 & & & 90 & $\begin{array}{l}\text { major vertical open } \\
\text { fracture }\end{array}$ \\
\hline & & 2.13 & 0.71 & -1.98 & N45E & SE & 70 & minor closed angled fracture \\
\hline & & 2.13 & 0.67 & -1.98 & N45E & SE & 70 & minor closed angled fracture \\
\hline \multicolumn{9}{|l|}{ NN3 } \\
\hline & & 2.13 & 2.91 & -3.81 & N45E & & 90 & $\begin{array}{l}\text { major open vertical } \\
\text { fracture }\end{array}$ \\
\hline & & 2.13 & 2.39 & -3.81 & NE-SW & & 90 & $\begin{array}{l}\text { major angled vertical } \\
\text { fracture }\end{array}$ \\
\hline & & 2.13 & 2.60 & -3.81 & NE-SW & & 90 & $\begin{array}{l}\text { major angled vertical } \\
\text { fracture }\end{array}$ \\
\hline & & $?$ & $?$ & $?$ & & & 90 & $\begin{array}{l}\text { major open vertical } \\
\text { fracture }\end{array}$ \\
\hline & & 2.13 & 1.75 & -3.81 & NW-SE & & & highly fractured zone \\
\hline & & 2.13 & 1.60 & -3.81 & NW-SE & & & highly fractured zone \\
\hline & & 2.13 & 1.60 & -3.81 & $E-W$ & & & $\begin{array}{l}\text { major(?) partly open } \\
\text { subhorizontal fracture }\end{array}$ \\
\hline & & 2.13 & 1.30 & -3.81 & $E-W$ & & & $\begin{array}{l}\text { major(?) partly open } \\
\text { subhorizontal fracture }\end{array}$ \\
\hline & LBT3 & 2.13 & 1.04 & -3.81 & $E-W$ & & & $\begin{array}{l}\text { major(?) subvertical open } \\
\text { fracture }\end{array}$ \\
\hline & & 2.13 & 1.04 & -3.81 & $E-W$ & $S$ & 15 & $\begin{array}{l}\text { major(?) continuous } \\
\text { horizontal(?) fracture }\end{array}$ \\
\hline & & 2.13 & 0.74 & -3.81 & $E-W$ & $S$ & 15 & $\begin{array}{l}\text { major(?) continuous } \\
\text { horizontal(?) fracture }\end{array}$ \\
\hline
\end{tabular}




\begin{tabular}{|c|c|c|c|c|c|c|c|c|}
\hline Hole & $\begin{array}{l}\text { Surface } \\
\text { Feature }\end{array}$ & $\begin{array}{c}\text { Model } \\
\text { East }\end{array}$ & $\begin{array}{l}\text { Model } \\
\text { North }\end{array}$ & $\begin{array}{l}\text { Model } \\
\text { Depth }\end{array}$ & $\begin{array}{l}\text { Strike } \\
\text { Direct }\end{array}$ & $\begin{array}{l}\text { Dip } \\
\text { Direct }\end{array}$ & Dip & Comments \\
\hline & & 2.13 & 0.84 & -3.81 & $E-W$ & $N$ & 15 & $\begin{array}{l}\text { major open subhorizontal } \\
\text { fracture }\end{array}$ \\
\hline & & 2.13 & 0.51 & -3.81 & $E-W$ & $\mathrm{~N}$ & 15 & $\begin{array}{l}\text { major open subhorizontal } \\
\text { fracture }\end{array}$ \\
\hline & & 2.13 & 0.51 & -3.81 & & & & $\begin{array}{l}\text { wandering minor } \\
\text { subhorizontal fracture }\end{array}$ \\
\hline & & 2.13 & 0.28 & -3.81 & & & & $\begin{array}{l}\text { wandering minor } \\
\text { subhorizontal fracture }\end{array}$ \\
\hline \multicolumn{9}{|l|}{ NN4 } \\
\hline & & 0.91 & 2.92 & -0.91 & NW-SE & $N E$ & & $\begin{array}{l}\text { moderate-major angled } \\
\text { fracture, open }\end{array}$ \\
\hline & & 0.91 & 2.84 & -0.91 & NE-SW & $\mathrm{MW}$ & & $\begin{array}{l}\text { moderate-major open angled } \\
\text { fracture }\end{array}$ \\
\hline & & 0.91 & 2.77 & -0.91 & NE-SW & $\mathrm{MW}$ & & $\begin{array}{l}\text { moderate-major open angled } \\
\text { fracture }\end{array}$ \\
\hline & & 0.91 & 2.77 & -0.91 & $E-W$ & & 90 & $\begin{array}{l}\text { moderate open wet vertical } \\
\text { fracture }\end{array}$ \\
\hline & & 0.91 & 2.31 & -0.91 & NW-SE & SW & & minor angled closed fracture \\
\hline & & 0.91 & 1.57 & -0.91 & NE-SW & SE & & $\begin{array}{l}\text { minor tight subvertical } \\
\text { fracture }\end{array}$ \\
\hline & & 0.91 & 1.50 & -0.91 & $\sim \mathrm{N}-\mathrm{S}$ & W & & $\begin{array}{l}\text { major open angled fracture, } \\
\text { trending along side of hole }\end{array}$ \\
\hline & & 0.91 & 1.09 & -0.91 & $\sim \mathrm{N}-\mathrm{S}$ & W & & $\begin{array}{l}\text { major open angled fracture, } \\
\text { trending along side of hole }\end{array}$ \\
\hline & LBT3 & 0.91 & 1.07 & -0.91 & & & 90 & $\begin{array}{l}\text { major open vertical wet } \\
\text { fracture }\end{array}$ \\
\hline & & 0.91 & 0.89 & -0.91 & $\sim \mathrm{N}-\mathrm{S}$ & W & & $\begin{array}{l}\text { moderate-major fracture } \\
\text { barely grazing side of hole }\end{array}$ \\
\hline & & 0.91 & 0.79 & -0.91 & $\sim \mathrm{N}-\mathrm{S}$ & W & & $\begin{array}{l}\text { moderate-major fracture } \\
\text { barely grazing side of hole }\end{array}$ \\
\hline & & 0.91 & 0.71 & -0.91 & NW-SE & SSW & & major angled open fracture \\
\hline & & 0.91 & 0.53 & -0.91 & NW-SE & SSW & & major angled open fracture \\
\hline & LBT2 & 0.91 & 0.41 & -0.91 & NW-SE & $N$ & & $\begin{array}{l}\text { moderate-major open angled } \\
\text { fracture }\end{array}$ \\
\hline \multicolumn{9}{|l|}{ NN5 } \\
\hline & & 0.91 & 2.64 & -1.98 & N45E & SE & 80 & major open fracture, angled \\
\hline & & 0.91 & 2.54 & -1.98 & $\mathrm{~N} 45 \mathrm{E}$ & SE & 80 & major open fracture, angled \\
\hline & & 0.91 & 2.54 & -1.98 & & & 90 & $\begin{array}{l}\text { major vertical fracture } \\
\text { trending along strike of } \\
\text { hole... }\end{array}$ \\
\hline & & 0.91 & 2.26 & -1.98 & & & 90 & major vertical fracture \\
\hline
\end{tabular}




\begin{tabular}{|c|c|c|c|c|c|c|c|c|}
\hline Hole & $\begin{array}{l}\text { Surface } \\
\text { Feature }\end{array}$ & $\begin{array}{l}\text { Model } \\
\text { East }\end{array}$ & $\begin{array}{l}\text { Model } \\
\text { North }\end{array}$ & $\begin{array}{l}\text { Model } \\
\text { Depth }\end{array}$ & $\begin{array}{l}\text { Strike } \\
\text { Direct }\end{array}$ & $\begin{array}{l}\text { Dip } \\
\text { Direct }\end{array}$ & Dip & Comments \\
\hline & & & & & & & & $\begin{array}{l}\text { trending along strike of } \\
\text { hole... }\end{array}$ \\
\hline & & 0.91 & 2.24 & -1.98 & NW-SE & $N E$ & & $\begin{array}{l}\text { moderate tight subvertical } \\
\text { partly open fracture }\end{array}$ \\
\hline & & 0.91 & 2.03 & -1.98 & NW-SE & $N E$ & & $\begin{array}{l}\text { moderate tight subvertical } \\
\text { partly open fracture }\end{array}$ \\
\hline & & 0.91 & 2.03 & -1.98 & $E-W$ & & 90 & $\begin{array}{l}\text { moderate vertical open } \\
\text { fracture }\end{array}$ \\
\hline & & 0.91 & 1.68 & -1.98 & $E-W$ & & & $\begin{array}{l}\text { minor tight subvertical } \\
\text { fracture }\end{array}$ \\
\hline & & 0.91 & 1.04 & -1.98 & $E-W$ & & 90 & $\begin{array}{l}\text { moderate-major open } \\
\text { vertical fracture }\end{array}$ \\
\hline & LBT2 & 0.91 & 0.94 & -1.98 & $\mathrm{~N} 45 \mathrm{~W}$ & $N E$ & 90 & $\begin{array}{l}\text { major open vertical } \\
\text { fracture }\end{array}$ \\
\hline & LBT2 & 0.91 & 0.94 & -1.98 & N45W & $N E$ & 90 & $\begin{array}{l}\text { major open vertical } \\
\text { fracture }\end{array}$ \\
\hline \multicolumn{9}{|l|}{ NN6 } \\
\hline & & 0.91 & 2.79 & -3.81 & N20E & & 90 & $\begin{array}{l}\text { angled tight fracture along } \\
\text { the wall surface } \\
\end{array}$ \\
\hline & & 0.91 & 2.67 & -3.81 & & & 90 & $\begin{array}{l}\text { minor vertical closed } \\
\text { fracture }\end{array}$ \\
\hline & & 0.91 & 2.54 & -3.81 & & & 90 & $\begin{array}{l}\text { minor vertical closed } \\
\text { fracture }\end{array}$ \\
\hline & & 0.91 & 2.26 & -3.81 & & & 90 & $\begin{array}{l}\text { major open vertical } \\
\text { fracture }\end{array}$ \\
\hline & & 0.91 & 2.07 & -3.81 & $\mathrm{~N}-\mathrm{S}$ & $\mathrm{E}$ & 45 & $\begin{array}{l}\text { open fracture parallels the } \\
\text { hole }\end{array}$ \\
\hline & & 0.91 & 1.96 & -3.81 & $\mathrm{~N}-\mathrm{S}$ & $E$ & 45 & $\begin{array}{l}\text { open fracture parallels the } \\
\text { hole }\end{array}$ \\
\hline & LBT2 & 0.91 & 1.63 & -3.81 & & & 90 & $\begin{array}{l}\text { major vertical(?) open } \\
\text { fracture (is grout exiting } \\
\text { fracture?) }\end{array}$ \\
\hline & & 0.91 & 1.57 & -3.81 & N45W & & & $\begin{array}{l}\text { major subvertical(?) angled } \\
\text { open fracture }\end{array}$ \\
\hline & & 0.91 & 1.24 & -3.81 & N30W & $\mathrm{MW}$ & 90 & angled major fracture \\
\hline & & 0.91 & 1.04 & -3.81 & N30W & $\mathrm{MW}$ & 90 & angled major fracture \\
\hline & & 0.91 & 1.14 & -3.81 & $E-W$ & S & 10 & $\begin{array}{l}\text { major subhorizontal open } \\
\text { fracture }\end{array}$ \\
\hline & & 0.91 & 0.81 & -3.81 & $E-W$ & $S$ & 10 & $\begin{array}{l}\text { major subhorizontal open } \\
\text { fracture }\end{array}$ \\
\hline & & 0.91 & 0.81 & -3.81 & $E-W$ & S & 20 & $\begin{array}{l}\text { minor wandering } \\
\text { subhorizontal fracture }\end{array}$ \\
\hline
\end{tabular}




\begin{tabular}{|c|c|c|c|c|c|c|c|c|}
\hline Hole & $\begin{array}{l}\text { Surface } \\
\text { Feature }\end{array}$ & $\begin{array}{c}\text { Model } \\
\text { East }\end{array}$ & $\begin{array}{l}\text { Model } \\
\text { North }\end{array}$ & $\begin{array}{l}\text { Model } \\
\text { Depth }\end{array}$ & $\begin{array}{l}\text { Strike } \\
\text { Direct }\end{array}$ & $\begin{array}{l}\text { Dip } \\
\text { Direct }\end{array}$ & Dip & Comments \\
\hline & & 0.91 & 0.51 & -3.81 & $E-W$ & $S$ & 20 & $\begin{array}{l}\text { minor wandering } \\
\text { subhorizontal fracture }\end{array}$ \\
\hline \multicolumn{9}{|l|}{ N01 } \\
\hline & & 2.13 & 2.64 & -4.11 & $E-W$ & $S$ & 90 & $\begin{array}{l}\text { moderate-major open to } \\
\text { partly open vertical } \\
\text { fracture }\end{array}$ \\
\hline & & 2.13 & 2.59 & -4.11 & $E-W$ & & 90 & $\begin{array}{l}\text { moderate-major open to } \\
\text { partly open vertical } \\
\text { fracture }\end{array}$ \\
\hline & & 2.13 & 2.08 & -4.11 & N70E & & 90 & angled major open fracture \\
\hline & & 2.13 & 2.04 & -4.11 & N70E & & 90 & angled major open fracture \\
\hline & & 2.13 & 1.74 & -4.11 & $E-W$ & & 90 & $\begin{array}{l}\text { series of significant open } \\
\text { fractures }\end{array}$ \\
\hline & & 2.13 & 1.60 & -4.11 & $E-W$ & & 90 & $\begin{array}{l}\text { series of significant open } \\
\text { fractures }\end{array}$ \\
\hline & & 2.13 & 1.45 & -4.11 & N20W & & 90 & angled large fracture \\
\hline & & 2.13 & 1.22 & -4.11 & N20W & & 90 & angled large fracture \\
\hline & LBT3 & 2.13 & 1.07 & -4.11 & & & 90 & $\begin{array}{l}\text { minor vertical fracture, } \\
\text { looks closed }\end{array}$ \\
\hline & & 2.13 & 0.89 & -4.11 & N20W & & 90 & $\begin{array}{l}\text { angled moderate-minor tight } \\
\text { closed fracture }\end{array}$ \\
\hline & & 2.13 & 0.79 & -4.11 & N20W & & 90 & $\begin{array}{l}\text { angled moderate-minor tight } \\
\text { closed fracture }\end{array}$ \\
\hline & & 2.13 & 0.61 & -4.11 & $\mathrm{~N} 30 \mathrm{E}$ & MW & & $\begin{array}{l}\text { angled minor tight closed } \\
\text { fracture }\end{array}$ \\
\hline & & 2.13 & 0.56 & -4.11 & $\mathrm{~N} 30 \mathrm{E}$ & $\mathrm{MW}$ & & $\begin{array}{l}\text { angled minor tight closed } \\
\text { fracture }\end{array}$ \\
\hline & & 2.13 & 0.53 & -4.11 & & & 90 & $\begin{array}{l}\text { minor vertical partly open } \\
\text { fracture }\end{array}$ \\
\hline & & 2.13 & 0.41 & -4.11 & $E-W$ & $S$ & & closed subvertical fracture \\
\hline & & 2.13 & 0.36 & -4.11 & $E-W$ & $S$ & & closed subvertical fracture \\
\hline \multicolumn{9}{|l|}{ N02 } \\
\hline & & 0.91 & 2.84 & -4.11 & $\mathrm{~N} 30 \mathrm{E}$ & SE & 30 & $\begin{array}{l}\text { complex series of } \\
\text { subhorizontal open fractures }\end{array}$ \\
\hline & & 0.91 & 2.50 & -4.11 & $\mathrm{~N} 30 \mathrm{E}$ & SE & 30 & $\begin{array}{l}\text { complex series of } \\
\text { subhorizontal open fractures }\end{array}$ \\
\hline & & 0.91 & 2.24 & -4.11 & N70E & & 90 & $\begin{array}{l}\text { significant open subvertical } \\
\text { fracture }\end{array}$ \\
\hline & & 0.91 & 1.73 & -4.11 & N60W & SW & 70 & minor angled closed fracture \\
\hline & & 0.91 & 1.68 & -4.11 & N60W & SW & 70 & minor angled closed fracture \\
\hline
\end{tabular}




\begin{tabular}{|c|c|c|c|c|c|c|c|c|}
\hline Hole & $\begin{array}{l}\text { Surface } \\
\text { Feature }\end{array}$ & $\begin{array}{c}\text { Model } \\
\text { East }\end{array}$ & $\begin{array}{l}\text { Model } \\
\text { North }\end{array}$ & $\begin{array}{l}\text { Model } \\
\text { Depth }\end{array}$ & $\begin{array}{l}\text { Strike } \\
\text { Direct }\end{array}$ & $\begin{array}{l}\text { Dip } \\
\text { Direct }\end{array}$ & Dip & Comments \\
\hline & & 0.91 & 1.55 & -4.11 & & & 90 & $\begin{array}{l}\text { major open vertical } \\
\text { fracture }\end{array}$ \\
\hline & & 0.91 & 1.40 & -4.11 & N30W & SW & 70 & minor angled closed fracture \\
\hline & & 0.91 & 1.30 & -4.11 & N30W & SW & 70 & minor angled closed fracture \\
\hline & & 0.91 & 1.30 & -4.11 & & & 90 & minor vertical fracture \\
\hline & & 0.91 & 1.30 & -4.11 & N30W & SW & 80 & $\begin{array}{l}\text { angled moderate-minor } \\
\text { partly open fracture }\end{array}$ \\
\hline & & 0.91 & 1.17 & -4.11 & N30W & SW & 80 & $\begin{array}{l}\text { angled moderate-minor } \\
\text { partly open fracture }\end{array}$ \\
\hline & & 0.91 & 1.14 & -4.11 & $\mathrm{~N} 30 \mathrm{E}$ & MW & & $\begin{array}{l}\text { angled minor-moderate } \\
\text { vertical fracture }\end{array}$ \\
\hline & & 0.91 & 0.97 & -4.11 & N30E & MW & & $\begin{array}{l}\text { angled minor-moderate } \\
\text { vertical fracture }\end{array}$ \\
\hline & & 0.91 & 0.97 & -4.11 & & & 90 & $\begin{array}{l}\text { moderate vertical partly } \\
\text { open fracture }\end{array}$ \\
\hline & & 0.91 & 0.64 & -4.11 & NE-SW & & 90 & $\begin{array}{l}\text { moderate vertical partly } \\
\text { open fracture }\end{array}$ \\
\hline & & 0.91 & 0.66 & -4.11 & $\sim \mathrm{N}-\mathrm{S}$ & & 90 & $\begin{array}{l}\text { major open angled vertical } \\
\text { fracture-wandering }\end{array}$ \\
\hline & & 0.91 & 0.43 & -4.11 & $\sim \mathrm{N}-\mathrm{S}$ & & 90 & $\begin{array}{l}\text { major open angled vertical } \\
\text { fracture-wandering }\end{array}$ \\
\hline & & 0.91 & 0.43 & -4.11 & $\mathrm{~N} 30 \mathrm{E}$ & SE & 80 & angled fracture \\
\hline & & 0.91 & 0.30 & -4.11 & $\mathrm{~N} 30 \mathrm{E}$ & SE & 80 & angled fracture \\
\hline \multicolumn{9}{|l|}{ NT1 } \\
\hline & LBT22 & 2.74 & 2.97 & -2.44 & $\mathrm{~N} 30 \mathrm{E}$ & MW & 70 & angled fracture \\
\hline & LBT22 & 2.74 & 2.84 & -2.44 & N30E & $\mathrm{MW}$ & 70 & angled fracture \\
\hline & & 2.74 & 2.74 & -2.44 & & & 90 & $\begin{array}{l}\text { moderate/major vertical } \\
\text { open fracture }\end{array}$ \\
\hline & & 2.74 & 2.26 & -2.44 & N70E & $\mathrm{MW}$ & 80 & angled minor fracture \\
\hline & & 2.74 & 2.24 & -2.44 & N70E & $\mathrm{MW}$ & 80 & angled minor fracture \\
\hline & & 2.74 & 2.13 & -2.44 & N70E & $\mathrm{MW}$ & 80 & angled minor fracture \\
\hline & & 2.74 & 2.11 & -2.44 & N70E & $\mathrm{MW}$ & 80 & angled minor fracture \\
\hline & & 2.74 & 2.13 & -2.44 & N60E & $\mathrm{MW}$ & 80 & angled minor fracture \\
\hline & & 2.74 & 2.06 & -2.44 & N60E & $\mathrm{MW}$ & 80 & angled minor fracture \\
\hline & & 2.74 & 2.11 & -2.44 & N30W & $\mathrm{MW}$ & 80 & $\begin{array}{l}\text { moderate-major open } \\
\text { fracture }\end{array}$ \\
\hline & & 2.74 & 2.01 & -2.44 & N30W & $M W$ & 80 & $\begin{array}{l}\text { moderate-major open } \\
\text { fracture }\end{array}$ \\
\hline & & 2.74 & 1.91 & -2.44 & NE-SW & MW & & minor tight angled fracture \\
\hline
\end{tabular}




\begin{tabular}{|c|c|c|c|c|c|c|c|c|}
\hline Hole & $\begin{array}{l}\text { Surface } \\
\text { Feature }\end{array}$ & $\begin{array}{c}\text { Model } \\
\text { East }\end{array}$ & $\begin{array}{l}\text { Model } \\
\text { North }\end{array}$ & $\begin{array}{l}\text { Model } \\
\text { Depth }\end{array}$ & $\begin{array}{l}\text { Strike } \\
\text { Direct }\end{array}$ & $\begin{array}{l}\text { Dip } \\
\text { Direct }\end{array}$ & Dip & Comments \\
\hline & & 2.74 & 1.88 & -2.44 & NE-SW & $\mathrm{MW}$ & & minor tight angled fracture \\
\hline & & 2.74 & 1.80 & -2.44 & & & 90 & $\begin{array}{l}\text { minor vertical tight } \\
\text { fracture }\end{array}$ \\
\hline & & 2.74 & 1.50 & -2.44 & $\mathrm{~N} 15 \mathrm{~W}$ & SW & 60 & major angled open fracture \\
\hline & & 2.74 & 1.24 & -2.44 & $\mathrm{~N} 15 \mathrm{~W}$ & SW & 60 & major angled open fracture \\
\hline & & 2.74 & 1.24 & -2.44 & & & 90 & $\begin{array}{l}\text { minor vertical partly open } \\
\text { fracture }\end{array}$ \\
\hline & & 2.74 & 1.09 & -2.44 & N45E & SE & 70 & $\begin{array}{l}\text { moderate partly open angled } \\
\text { fracture }\end{array}$ \\
\hline & & 2.74 & 1.03 & -2.44 & N45E & SE & 70 & $\begin{array}{l}\text { moderate partly open angled } \\
\text { fracture }\end{array}$ \\
\hline & LBT3 & 2.74 & 0.94 & -2.44 & & & 90 & $\begin{array}{l}\text { moderate open vertical wet } \\
\text { fracture }\end{array}$ \\
\hline \multicolumn{9}{|l|}{ NT2 } \\
\hline & & 0.91 & 2.41 & -0.61 & NW-SE & & & $\begin{array}{l}\text { minor tight subvertical } \\
\text { fracture }\end{array}$ \\
\hline & & 0.91 & 2.31 & -0.61 & & & 90 & $\begin{array}{l}\text { minor tight vertical } \\
\text { fracture }\end{array}$ \\
\hline & & 0.91 & 1.55 & -0.61 & NW-SE & SW & & moderate open fracture \\
\hline & & 0.91 & 1.32 & -0.61 & NW-SE & SW & & moderate open fracture \\
\hline & & 0.91 & 1.09 & -0.61 & $E-W$ & & 90 & $\begin{array}{l}\text { minor vertical generally } \\
\text { closed fracture }\end{array}$ \\
\hline & & 0.91 & 1.07 & -0.61 & $E-W$ & & 90 & $\begin{array}{l}\text { minor vertical generally } \\
\text { closed fracture }\end{array}$ \\
\hline & & 0.91 & 0.69 & -0.61 & & & 90 & $\begin{array}{l}\text { minor vertical closed } \\
\text { fracture }\end{array}$ \\
\hline \multicolumn{9}{|l|}{ NT3 } \\
\hline & & 0.91 & 2.72 & -3.20 & & & 90 & $\begin{array}{l}\text { tight, minor vertical } \\
\text { fracture }\end{array}$ \\
\hline & & 0.91 & 2.79 & -3.20 & N30W & $N E$ & 80 & $\begin{array}{l}\text { minor, subvertical fracture, } \\
\text { angled }\end{array}$ \\
\hline & & 0.91 & 2.72 & -3.20 & $E-W$ & W & & $\begin{array}{l}\text { minor, subvertical fracture, } \\
\text { angled }\end{array}$ \\
\hline & & 0.91 & 2.41 & -3.20 & $E-W$ & & 90 & $\begin{array}{l}\text { major, open vertical } \\
\text { fracture }\end{array}$ \\
\hline & & 0.91 & 1.85 & -3.20 & N45E & $M W$ & & $\begin{array}{l}\text { minor-moderate subvertical } \\
\text { fracture, partly open }\end{array}$ \\
\hline & & 0.91 & 1.73 & -3.20 & N45E & $M W$ & & $\begin{array}{l}\text { minor-moderate subvertical } \\
\text { fracture, partly open }\end{array}$ \\
\hline & & 0.91 & 1.73 & -3.20 & $E-W$ & & 90 & $\begin{array}{l}\text { major open vertical } \\
\text { fracture }\end{array}$ \\
\hline
\end{tabular}




\begin{tabular}{|c|c|c|c|c|c|c|c|c|}
\hline Hole & $\begin{array}{l}\text { Surface } \\
\text { Feature }\end{array}$ & $\begin{array}{l}\text { Model } \\
\text { East }\end{array}$ & $\begin{array}{l}\text { Model } \\
\text { North }\end{array}$ & $\begin{array}{l}\text { Model } \\
\text { Depth }\end{array}$ & $\begin{array}{l}\text { Strike } \\
\text { Direct }\end{array}$ & $\begin{array}{l}\text { Dip } \\
\text { Direct }\end{array}$ & Dip & Comments \\
\hline & & 0.91 & 1.52 & -3.20 & $E-W$ & SE/S & & $\begin{array}{l}\text { moderate-major open } \\
\text { subvertical fracture }\end{array}$ \\
\hline & & 0.91 & 1.35 & -3.20 & NW-SE & SW & 90 & $\begin{array}{l}\text { moderate-major open } \\
\text { vertical fracture }\end{array}$ \\
\hline & & 0.91 & 1.17 & -3.20 & & & & $\begin{array}{l}\text { minor subvertical closed } \\
\text { fracture }\end{array}$ \\
\hline & & 0.91 & 0.97 & -3.20 & $E-W$ & & 90 & $\begin{array}{l}\text { moderate vertical fracture, } \\
\text { partly open }\end{array}$ \\
\hline \multicolumn{9}{|l|}{ NT4 } \\
\hline & LBT24 & 0.30 & 2.46 & -2.44 & NE-SW & $\mathrm{MW}$ & 70 & $\begin{array}{l}\text { major-moderate angled } \\
\text { sharp open fracture }\end{array}$ \\
\hline & LBT24 & 0.30 & 2.41 & -2.44 & NE-SW & $M W$ & 70 & $\begin{array}{l}\text { major-moderate angled } \\
\text { sharp open fracture }\end{array}$ \\
\hline & & 0.30 & 2.44 & -2.44 & NE-SW & $M W$ & 70 & $\begin{array}{l}\text { major-moderate angled } \\
\text { sharp open fracture }\end{array}$ \\
\hline & & 0.30 & 2.39 & -2.44 & NE-SW & $M W$ & 70 & $\begin{array}{l}\text { major-moderate angled } \\
\text { sharp open fracture }\end{array}$ \\
\hline & & 0.30 & 2.39 & -2.44 & NE-SW & $\mathrm{MW}$ & 70 & $\begin{array}{l}\text { major-moderate angled } \\
\text { sharp open fracture }\end{array}$ \\
\hline & & 0.30 & 2.34 & -2.44 & NE-SW & $M W$ & 70 & $\begin{array}{l}\text { major-moderate angled } \\
\text { sharp open fracture }\end{array}$ \\
\hline & & 0.30 & 2.03 & -2.44 & NE-SW & $M W$ & 80 & $\begin{array}{l}\text { moderate angled partly open } \\
\text { fracture }\end{array}$ \\
\hline & & 0.30 & 2.01 & -2.44 & NE-SW & $M W$ & 80 & $\begin{array}{l}\text { moderate angled partly open } \\
\text { fracture }\end{array}$ \\
\hline & & 0.30 & 1.98 & -2.44 & N80E & & & $\begin{array}{l}\text { major open vertical- } \\
\text { subvertical fracture }\end{array}$ \\
\hline & & 0.30 & 1.85 & -2.44 & N20E & $M W$ & 45 & $\begin{array}{l}\text { moderate-major discrete } \\
\text { sharp partly open/closed }\end{array}$ \\
\hline & & 0.30 & 1.68 & -2.44 & N20E & $M W$ & 45 & $\begin{array}{l}\text { moderate-major discrete } \\
\text { sharp partly open/closed }\end{array}$ \\
\hline & LBT23 & 0.30 & 1.35 & -2.44 & NE-SW & $\mathrm{MW}$ & 45 & major partly open fracture \\
\hline & LBT23 & 0.30 & 0.89 & -2.44 & NE-SW & $\mathrm{MW}$ & 45 & major partly open fracture \\
\hline & LBT3 & 0.30 & 1.02 & -2.44 & & & 90 & $\begin{array}{l}\text { moderate open vertical } \\
\text { fracture }\end{array}$ \\
\hline & & 0.30 & 0.86 & -2.44 & NW-SE & SW & 55 & $\begin{array}{l}\text { minor tight/healed angled } \\
\text { fracture }\end{array}$ \\
\hline & & 0.30 & 0.71 & -2.44 & NW-SE & SW & 55 & $\begin{array}{l}\text { minor tight/healed angled } \\
\text { fracture }\end{array}$ \\
\hline
\end{tabular}




\begin{tabular}{|c|c|c|c|c|c|c|c|c|}
\hline Hole & $\begin{array}{l}\text { Surface } \\
\text { Feature }\end{array}$ & $\begin{array}{c}\text { Model } \\
\text { East }\end{array}$ & $\begin{array}{l}\text { Model } \\
\text { North }\end{array}$ & $\begin{array}{l}\text { Model } \\
\text { Depth }\end{array}$ & $\begin{array}{l}\text { Strike } \\
\text { Direct }\end{array}$ & $\begin{array}{l}\text { Dip } \\
\text { Direct }\end{array}$ & Dip & Comments \\
\hline \multicolumn{9}{|l|}{ WH1 } \\
\hline & LBT16(?) & 0.66 & 1.68 & -1.22 & & & 90 & $\begin{array}{l}\text { major open vertical } \\
\text { fracture }\end{array}$ \\
\hline & & 1.09 & 1.68 & -1.22 & SW-NE & $\mathrm{E} ?$ & & $\begin{array}{l}\text { angled minor/moderate } \\
\text { subhorizontal party open } \\
\text { fracture }\end{array}$ \\
\hline & & 1.78 & 1.68 & -1.22 & & & 90 & $\begin{array}{l}\text { minor vertical partly open } \\
\text { fracture }\end{array}$ \\
\hline & & 2.08 & 1.68 & -1.22 & $\sim \mathrm{SW}-\mathrm{NE}$ & $E$ & & $\begin{array}{l}\text { moderate subhorizontal } \\
\text { closed fracture }\end{array}$ \\
\hline & & 2.18 & 1.68 & -1.22 & $\sim \mathrm{SW}-\mathrm{NE}$ & E & & $\begin{array}{l}\text { moderate subhorizontal } \\
\text { closed fracture }\end{array}$ \\
\hline \multicolumn{9}{|l|}{ WH2 } \\
\hline & & 0.48 & 1.68 & -2.29 & & & 90 & $\begin{array}{l}\text { minor tight vertical } \\
\text { fracture }\end{array}$ \\
\hline & & 0.69 & 1.68 & -2.29 & NE-SW & & 90 & $\begin{array}{l}\text { minor tight vertical } \\
\text { fracture }\end{array}$ \\
\hline & & 1.27 & 1.68 & -2.29 & & & 90 & $\begin{array}{l}\text { minor tight vertical } \\
\text { fracture }\end{array}$ \\
\hline & & 1.47 & 1.68 & -2.29 & $\mathrm{~N}-\mathrm{S}$ & & 90 & $\begin{array}{l}\text { major open wet vertical } \\
\text { fracture }\end{array}$ \\
\hline & & 1.83 & 1.68 & -2.29 & & & 90 & $\begin{array}{l}\text { minor tight vertical } \\
\text { fracture }\end{array}$ \\
\hline & & 1.98 & 1.68 & -2.29 & & & 90 & $\begin{array}{l}\text { minor tight vertical } \\
\text { fracture }\end{array}$ \\
\hline & & 2.41 & 1.68 & -2.29 & NW-SE & SW & & moderate tight (?) fracture \\
\hline & & 2.77 & 1.68 & -2.29 & NW-SE & SW & & moderate tight (?) fracture \\
\hline & & 2.49 & 1.68 & -2.29 & & & 90 & $\begin{array}{l}\text { minor tight vertical } \\
\text { fracture }\end{array}$ \\
\hline \multicolumn{9}{|l|}{ WM1 } \\
\hline & & 1.78 & 2.13 & -3.96 & & E-NE & & $\begin{array}{l}\text { major(?) subhorizontal } \\
\text { fracture, partly open }\end{array}$ \\
\hline & & 2.13 & 2.13 & -3.96 & & $\mathrm{E}-\mathrm{NE}$ & & $\begin{array}{l}\text { major(?) subhorizontal } \\
\text { fracture, partly open }\end{array}$ \\
\hline & & 2.67 & 2.13 & -3.96 & & & 90 & $\begin{array}{l}\text { moderate-major vertical } \\
\text { open fracture }\end{array}$ \\
\hline & & 2.44 & 2.13 & -3.96 & & & 90 & $\begin{array}{l}\text { minor vertical closed } \\
\text { fracture }\end{array}$ \\
\hline & & 2.34 & 2.13 & -3.96 & & & 90 & $\begin{array}{l}\text { major vertical open } \\
\text { fracture }\end{array}$ \\
\hline
\end{tabular}




\begin{tabular}{|c|c|c|c|c|c|c|c|c|}
\hline Hole & $\begin{array}{l}\text { Surface } \\
\text { Feature }\end{array}$ & $\begin{array}{c}\text { Model } \\
\text { East }\end{array}$ & $\begin{array}{l}\text { Model } \\
\text { North }\end{array}$ & $\begin{array}{l}\text { Model } \\
\text { Depth }\end{array}$ & $\begin{array}{l}\text { Strike } \\
\text { Direct }\end{array}$ & $\begin{array}{l}\text { Dip } \\
\text { Direct }\end{array}$ & Dip & Comments \\
\hline & & 1.55 & 2.13 & -3.96 & & & 90 & $\begin{array}{l}\text { major open vertical } \\
\text { fracture }\end{array}$ \\
\hline & & 1.50 & 2.13 & -3.96 & & & 90 & $\begin{array}{l}\text { major open vertical } \\
\text { fracture }\end{array}$ \\
\hline & & 1.55 & 2.13 & -3.96 & & & & $\begin{array}{l}\text { series of generally minor } \\
\text { vertical to subvertical tight } \\
\text { fractures }\end{array}$ \\
\hline & & 1.09 & 2.13 & -3.96 & & & & $\begin{array}{l}\text { series of generally minor } \\
\text { vertical to subvertical tight } \\
\text { fractures }\end{array}$ \\
\hline & & 0.81 & 2.13 & -3.96 & NE-SW & SW & & $\begin{array}{l}\text { moderate partly open } \\
\text { subvertical fracture }\end{array}$ \\
\hline & LBT2 & 0.53 & 2.13 & -3.96 & $\mathrm{~N}-\mathrm{S}$ & & 90 & minor vertical fracture \\
\hline & & 0.15 & 2.13 & -3.96 & & & 90 & $\begin{array}{l}\text { moderate-major vertical } \\
\text { open fracture }\end{array}$ \\
\hline \multicolumn{9}{|l|}{ WM1? } \\
\hline & & 0.43 & 2.13 & -3.96 & & & & $\begin{array}{l}\text { minor tight } \\
\text { subvertical/vertical } \\
\text { fracture }\end{array}$ \\
\hline & & 0.66 & 2.13 & -3.96 & $\mathrm{~N}-\mathrm{S}$ & & 90 & $\begin{array}{l}\text { minor tight vertical } \\
\text { fracture }\end{array}$ \\
\hline & & 1.09 & 2.13 & -3.96 & & & 90 & $\begin{array}{l}\text { minor vertical tight } \\
\text { fracture }\end{array}$ \\
\hline & & 1.35 & 2.13 & -3.96 & & & 90 & $\begin{array}{l}\text { minor vertical tight } \\
\text { fracture }\end{array}$ \\
\hline & & 1.77 & 2.13 & -3.96 & & & 90 & $\begin{array}{l}\text { minor vertical tight } \\
\text { fracture }\end{array}$ \\
\hline & & 2.06 & 2.13 & -3.96 & & & 90 & $\begin{array}{l}\text { minor vertical tight } \\
\text { fracture }\end{array}$ \\
\hline & & 2.34 & 2.13 & -3.96 & $\mathrm{~N} 45 \mathrm{~W}$ & SW & 70 & angled major open fracture \\
\hline & & 2.41 & 2.13 & -3.96 & $\mathrm{~N} 45 \mathrm{~W}$ & SW & 70 & angled major open fracture \\
\hline & & 2.46 & 2.13 & -3.96 & & & & $\begin{array}{l}\text { major open } \\
\text { vertical/subvertical } \\
\text { fracture-rubbly }\end{array}$ \\
\hline & & 2.46 & 2.13 & -3.96 & & & & $\begin{array}{l}\text { major series of horizontal } \\
\text { to subhorizontal open } \\
\text { fractures }\end{array}$ \\
\hline & & 2.77 & 2.13 & -3.96 & & & & $\begin{array}{l}\text { major series of horizontal } \\
\text { to subhorizontal open } \\
\text { fractures }\end{array}$ \\
\hline
\end{tabular}




\begin{tabular}{|c|c|c|c|c|c|c|c|c|}
\hline Hole & $\begin{array}{l}\text { Surface } \\
\text { Feature }\end{array}$ & $\begin{array}{l}\text { Model } \\
\text { East }\end{array}$ & $\begin{array}{l}\text { Model } \\
\text { North }\end{array}$ & $\begin{array}{l}\text { Model } \\
\text { Depth }\end{array}$ & $\begin{array}{l}\text { Strike } \\
\text { Direct }\end{array}$ & $\begin{array}{l}\text { Dip } \\
\text { Direct }\end{array}$ & Dip & Comments \\
\hline \multicolumn{9}{|l|}{ WM2 } \\
\hline & LBT2 & 0.66 & 0.91 & -1.22 & & & & $\begin{array}{l}\text { moderate subhorizontal } \\
\text { fracture-hard to see }\end{array}$ \\
\hline & & 0.97 & 0.91 & -1.22 & N20W & $N E$ & 20 & $\begin{array}{l}\text { major subhorizontal closed } \\
\text { to open fracture }\end{array}$ \\
\hline & & 1.52 & 0.91 & -1.22 & N20W & $N E$ & 20 & $\begin{array}{l}\text { major subhorizontal closed } \\
\text { to open fracture }\end{array}$ \\
\hline & & 1.80 & 0.91 & -1.22 & & & & $\begin{array}{l}\text { minor mostly closed } \\
\text { vertical fracture }\end{array}$ \\
\hline & & 2.08 & 0.91 & -1.22 & & & 90 & $\begin{array}{l}\text { minor vertical closed } \\
\text { fracture }\end{array}$ \\
\hline & & 2.41 & 0.91 & -1.22 & & & 90 & $\begin{array}{l}\text { minor vertical closed } \\
\text { fracture }\end{array}$ \\
\hline WM3 & \multicolumn{3}{|c|}{ cannot see the depths on tape } & & & & & \\
\hline \multicolumn{9}{|l|}{ WN2 } \\
\hline & & 0.10 & 2.13 & -1.68 & $\mathrm{~N} 30 \mathrm{E}$ & SE & 70 & $\begin{array}{l}\text { angled minor closed/healed } \\
\text { subvertical fracture }\end{array}$ \\
\hline & & 0.13 & 2.13 & -1.68 & $\mathrm{~N} 30 \mathrm{E}$ & SE & 70 & $\begin{array}{l}\text { angled minor closed/healed } \\
\text { subvertical fracture }\end{array}$ \\
\hline & & 0.20 & 2.13 & -1.68 & & & 90 & minor vertical fracture \\
\hline & & 0.51 & 2.13 & -1.68 & $\sim N-S$ & W & 80 & $\begin{array}{l}\text { major open fracture, with } \\
\text { some associated fractures }\end{array}$ \\
\hline & & 0.53 & 2.13 & -1.68 & $\sim \mathrm{N}-\mathrm{S}$ & W & 80 & $\begin{array}{l}\text { major open fracture, with } \\
\text { some associated fractures }\end{array}$ \\
\hline & & 0.84 & 2.13 & -1.68 & $\mathrm{~N} 45 \mathrm{~W}$ & SW & 40 & $\begin{array}{l}\text { major angled open to partly } \\
\text { open fracture }\end{array}$ \\
\hline & & 0.66 & 2.13 & -1.68 & $\mathrm{~N} 45 \mathrm{~W}$ & SW & 40 & $\begin{array}{l}\text { major angled open to partly } \\
\text { open fracture }\end{array}$ \\
\hline & & 1.04 & 2.13 & -1.68 & & & 90 & $\begin{array}{l}\text { minor vertical closed } \\
\text { fracture }\end{array}$ \\
\hline & & 1.24 & 2.13 & -1.68 & & & 90 & $\begin{array}{l}\text { minor vertical closed } \\
\text { fracture }\end{array}$ \\
\hline & & 1.27 & 2.13 & -1.68 & $\mathrm{~N}-\mathrm{S}$ & W & 20 & $\begin{array}{l}\text { major/moderate angled long } \\
\text { fracture, healed }\end{array}$ \\
\hline & LBT12(?) & 1.55 & 2.13 & -1.68 & $\mathrm{~N}-\mathrm{S}$ & W & 20 & $\begin{array}{l}\text { major/moderate angled long } \\
\text { fracture, healed }\end{array}$ \\
\hline & & 1.47 & 2.13 & -1.68 & & & 90 & $\begin{array}{l}\text { major open vertical } \\
\text { fracture }\end{array}$ \\
\hline & & 1.65 & 2.13 & -1.68 & & & 90 & $\begin{array}{l}\text { moderate vertical partly } \\
\text { open fracture (displaced by } \\
\text { a fault) }\end{array}$ \\
\hline
\end{tabular}




\begin{tabular}{|c|c|c|c|c|c|c|c|c|}
\hline Hole & $\begin{array}{l}\text { Surface } \\
\text { Feature }\end{array}$ & $\begin{array}{c}\text { Model } \\
\text { East }\end{array}$ & $\begin{array}{l}\text { Model } \\
\text { North }\end{array}$ & $\begin{array}{l}\text { Model } \\
\text { Depth }\end{array}$ & $\begin{array}{l}\text { Strike } \\
\text { Direct }\end{array}$ & $\begin{array}{l}\text { Dip } \\
\text { Direct }\end{array}$ & Dip & Comments \\
\hline & LBT13(?) & 1.80 & 2.13 & -1.68 & N80W & SW & 70 & $\begin{array}{l}\text { major? long angled } \\
\text { fracture/fault? }\end{array}$ \\
\hline & LBT13(?) & 1.55 & 2.13 & -1.68 & N80W & SW & 70 & $\begin{array}{l}\text { major? long angled } \\
\text { fracture/fault? }\end{array}$ \\
\hline & & 1.75 & 2.13 & -1.68 & & & 90 & $\begin{array}{l}\text { minor vertical fracture } \\
\text { (displaced by fault) }\end{array}$ \\
\hline & & 2.03 & 2.13 & -1.68 & & & 90 & $\begin{array}{l}\text { minor vertical closed } \\
\text { fracture }\end{array}$ \\
\hline & & 2.29 & 2.13 & -1.68 & & & 90 & $\begin{array}{l}\text { moderate vertical sharp } \\
\text { open fracture }\end{array}$ \\
\hline & & 2.62 & 2.13 & -1.68 & & & & $\begin{array}{l}\text { minor subvertical closed } \\
\text { fracture }\end{array}$ \\
\hline \multicolumn{9}{|l|}{ WN3 } \\
\hline & LBT2 & 0.88 & 1.68 & -3.96 & & & 90 & $\begin{array}{l}\text { major vertical open wet } \\
\text { fracture }\end{array}$ \\
\hline & & 1.00 & 1.68 & -3.96 & & & 90 & $\begin{array}{l}\text { Sharp open wet vertical } \\
\text { moderate fracture }\end{array}$ \\
\hline & & 1.21 & 1.68 & -3.96 & & & 90 & $\begin{array}{l}\text { major open wet vertical } \\
\text { fracture }\end{array}$ \\
\hline & & 1.35 & 1.68 & -3.96 & & & 90 & $\begin{array}{l}\text { major open wet vertical } \\
\text { fracture }\end{array}$ \\
\hline & & 2.77 & 1.68 & -3.96 & & & 90 & $\begin{array}{l}\text { vertical fracture-gaping } \\
\text { hole }\end{array}$ \\
\hline & & 1.30 & 1.68 & -3.96 & N70E & SE & 80 & top sharp open fracture \\
\hline & & 1.50 & 1.68 & -3.96 & N70E & SE & 80 & base sharp open fracture \\
\hline & & 1.50 & 1.68 & -3.96 & N60W & W & 45 & $\begin{array}{l}\text { top moderate partially open } \\
\text { fracture (shallow) }\end{array}$ \\
\hline & & 1.63 & 1.68 & -3.96 & N60W & W & 45 & $\begin{array}{l}\text { base moderate partially } \\
\text { open fracture (shallow) }\end{array}$ \\
\hline & & 1.55 & 1.68 & -3.96 & & W & & $\begin{array}{l}\text { subvertical partially open } \\
\text { fracture }\end{array}$ \\
\hline & & 1.88 & 1.68 & -3.96 & & & & $\begin{array}{l}\text { subvertical moderate wet } \\
\text { open fracture }\end{array}$ \\
\hline & & 1.96 & 1.68 & -3.96 & N70W & SW & 80 & $\begin{array}{l}\text { top major partly open angled } \\
\text { fracture }\end{array}$ \\
\hline & & 2.34 & 1.68 & -3.96 & N70W & SW & 80 & $\begin{array}{l}\text { base major partly open } \\
\text { angled fracture }\end{array}$ \\
\hline & & 2.11 & 1.68 & -3.96 & & & & $\begin{array}{l}\text { major subvertical open wet } \\
\text { fracture }\end{array}$ \\
\hline & & 2.34 & 1.68 & -3.96 & & & & $\begin{array}{l}\text { major subvertical open wet } \\
\text { fracture }\end{array}$ \\
\hline
\end{tabular}




\begin{tabular}{|c|c|c|c|c|c|c|c|c|}
\hline Hole & $\begin{array}{l}\text { Surface } \\
\text { Feature }\end{array}$ & $\begin{array}{c}\text { Model } \\
\text { East }\end{array}$ & $\begin{array}{l}\text { Model } \\
\text { North }\end{array}$ & $\begin{array}{l}\text { Model } \\
\text { Depth }\end{array}$ & $\begin{array}{l}\text { Strike } \\
\text { Direct }\end{array}$ & $\begin{array}{l}\text { Dip } \\
\text { Direct }\end{array}$ & Dip & Comments \\
\hline & & 2.55 & 1.68 & -3.96 & $\mathrm{~N} 45 \mathrm{~W}$ & SW & 70 & top tight closed fracture \\
\hline & & 2.59 & 1.68 & -3.96 & $\mathrm{~N} 45 \mathrm{~W}$ & SW & 70 & base tight closed fracture \\
\hline & & 2.67 & 1.68 & -3.96 & & & & $\begin{array}{l}\text { top subvertical wet open } \\
\text { fracture }\end{array}$ \\
\hline & & 2.69 & 1.68 & -3.96 & & & & $\begin{array}{l}\text { base subvertical wet open } \\
\text { fracture }\end{array}$ \\
\hline \multicolumn{9}{|l|}{ WN4 } \\
\hline & & 0.56 & 0.91 & -1.68 & & & 90 & $\begin{array}{l}\text { minor sharp vertical } \\
\text { fracture, thin maybe partly } \\
\text { open }\end{array}$ \\
\hline & & 0.76 & 0.91 & -1.68 & NW-SE & $\mathrm{NE}$ & & major partly open fracture \\
\hline & LBT2 & 0.80 & 0.91 & -1.68 & NW-SE & NE & & major partly open fracture \\
\hline & & 1.50 & 0.91 & -1.68 & $\mathrm{~N}-\mathrm{S}$ & S & 45 & $\begin{array}{l}\text { major partly open angled } \\
\text { fracture }\end{array}$ \\
\hline & & 1.57 & 0.91 & -1.68 & $\mathrm{~N}-\mathrm{S}$ & $S$ & 45 & $\begin{array}{l}\text { major partly open angled } \\
\text { fracture }\end{array}$ \\
\hline & & 1.70 & 0.91 & -1.68 & & & 90 & $\begin{array}{l}\text { moderate vertical open } \\
\text { fracture }\end{array}$ \\
\hline & & 1.75 & 0.91 & -1.68 & $\mathrm{~N}-\mathrm{S}$ & & 90 & minor vertical fracture \\
\hline & & 1.78 & 0.91 & -1.68 & $\mathrm{~N}-\mathrm{S}$ & & 90 & minor vertical fracture \\
\hline & & 2.01 & 0.91 & -1.68 & & & 90 & $\begin{array}{l}\text { vertical mostly closed } \\
\text { fracture }\end{array}$ \\
\hline & & 2.03 & 0.91 & -1.68 & & & 90 & $\begin{array}{l}\text { minor tight vertical } \\
\text { fracture }\end{array}$ \\
\hline & & 2.31 & 0.91 & -1.68 & & & 90 & $\begin{array}{l}\text { moderate sharp, vertical } \\
\text { open fracture, wet? }\end{array}$ \\
\hline \multicolumn{9}{|l|}{ W05 } \\
\hline & & 0.09 & 0.91 & -3.96 & & & 90 & $\begin{array}{l}\text { vertical moderate wet open } \\
\text { fracture }\end{array}$ \\
\hline & & 0.10 & 0.91 & -3.96 & $\mathrm{~N}-\mathrm{S}$ & W & & top shallow tight fracture \\
\hline & & 0.14 & 0.91 & -3.96 & N05E & $\mathrm{MW}$ & 20 & $\begin{array}{l}\text { base major open angled wet } \\
\text { fracture }\end{array}$ \\
\hline & & 0.25 & 0.91 & -3.96 & N05E & $\mathrm{MW}$ & 20 & $\begin{array}{l}\text { top major open angled wet } \\
\text { fracture }\end{array}$ \\
\hline & & 0.42 & 0.91 & -3.96 & NW-SE & & & $\begin{array}{l}\text { generally minor subvertical } \\
\text { tight fracture }\end{array}$ \\
\hline & & 0.55 & 0.91 & -3.96 & NE-SW & MW & & $\begin{array}{l}\text { base angled tight fracture } \\
\text { (top not seen) }\end{array}$ \\
\hline & & 0.58 & 0.91 & -3.96 & N30W & $\mathrm{NE}$ & 80 & moderate wet open fracture \\
\hline & & 0.61 & 0.91 & -3.96 & N30W & $\mathrm{NE}$ & 80 & moderate wet open fracture \\
\hline
\end{tabular}




\begin{tabular}{|c|c|c|c|c|c|c|c|c|}
\hline Hole & $\begin{array}{l}\text { Surface } \\
\text { Feature }\end{array}$ & $\begin{array}{c}\text { Model } \\
\text { East }\end{array}$ & $\begin{array}{l}\text { Model } \\
\text { North }\end{array}$ & $\begin{array}{l}\text { Model } \\
\text { Depth }\end{array}$ & $\begin{array}{l}\text { Strike } \\
\text { Direct }\end{array}$ & $\begin{array}{l}\text { Dip } \\
\text { Direct }\end{array}$ & Dip & Comments \\
\hline & & 0.61 & 0.91 & -3.96 & $\mathrm{~N}-\mathrm{S}$ & & & $\begin{array}{l}\text { subvertical tight minor } \\
\text { fracture }\end{array}$ \\
\hline & & 0.64 & 0.91 & -3.96 & $\sim E-W$ & & 90 & $\begin{array}{l}\text { base major open angled } \\
\text { fracture wet alteration halo }\end{array}$ \\
\hline & & 1.17 & 0.91 & -3.96 & $\sim E-W$ & & & $\begin{array}{l}\text { top major wandering } \\
\text { subvertical fracture }\end{array}$ \\
\hline & & 0.72 & 0.91 & -3.96 & $\mathrm{~N}-\mathrm{S} ?$ & & & $\begin{array}{l}\text { partly open wet minor } \\
\text { subvertical fracture on } \\
\text { north side of hole }\end{array}$ \\
\hline & & 0.79 & 0.91 & -3.96 & & & & $\begin{array}{l}\text { major? open wet } \\
\text { subvertical fracture }\end{array}$ \\
\hline & & 0.93 & 0.91 & -3.96 & $\mathrm{~N}-\mathrm{S}$ & & & $\begin{array}{l}\text { major? open wet } \\
\text { subvertical fracture }\end{array}$ \\
\hline & & 0.99 & 0.91 & -3.96 & $\mathrm{~N}-\mathrm{S} ?$ & & & $\begin{array}{l}\text { minor wet partly open } \\
\text { fracture subvertical on } \\
\text { north side of hole }\end{array}$ \\
\hline & & 1.08 & 0.91 & -3.96 & $N-S$ & & & $\begin{array}{l}\text { moderate partly open wet } \\
\text { subvertical fracture }\end{array}$ \\
\hline & & 1.46 & 0.91 & -3.96 & & & 90 & $\begin{array}{l}\text { fracture zone wet open } \\
\text { some vertical and angled }\end{array}$ \\
\hline & & 1.32 & 0.91 & -3.96 & & & 90 & $\begin{array}{l}\text { fracture zone wet open } \\
\text { some vertical and angled }\end{array}$ \\
\hline & & 1.60 & 0.91 & -3.96 & & & & $\begin{array}{l}\text { subvertical major? open? } \\
\text { fracture along the north } \\
\text { wall }\end{array}$ \\
\hline & & 1.52 & 0.91 & -3.96 & & & & $\begin{array}{l}\text { subvertical major? open? } \\
\text { fracture along the north } \\
\text { wall }\end{array}$ \\
\hline & & 1.63 & 0.91 & -3.96 & & & & $\begin{array}{l}\text { small patch of same } \\
\text { subvertical major? open? } \\
\text { fracture along the north } \\
\text { wall }\end{array}$ \\
\hline & & 1.73 & 0.91 & -3.96 & $N-S$ & & & $\begin{array}{l}\text { tight subvertical fracture } \\
\text { mainly on the north side of } \\
\text { hole }\end{array}$ \\
\hline & & 1.84 & 0.91 & -3.96 & $\mathrm{~N} 45 \mathrm{~W}$ & SW & 45 & tight minor angled fracture \\
\hline & & 1.91 & 0.91 & -3.96 & $\mathrm{~N} 45 \mathrm{~W}$ & SW & 45 & tight minor angled fracture \\
\hline & & 1.88 & 0.91 & -3.96 & $\mathrm{~N}-\mathrm{S}$ & & & $\begin{array}{l}\text { subvertical partly open wet } \\
\text { fracture }\end{array}$ \\
\hline & & 2.25 & 0.91 & -3.96 & $N-S$ & & & $\begin{array}{l}\text { major open wet subvertical } \\
\text { fracture }\end{array}$ \\
\hline & & 2.32 & 0.91 & -3.96 & $\mathrm{~N}-\mathrm{S}$ & & & $\begin{array}{l}\text { major open wet subvertical } \\
\text { fracture }\end{array}$ \\
\hline
\end{tabular}




\begin{tabular}{|c|c|c|c|c|c|c|c|c|}
\hline Hole & $\begin{array}{l}\text { Surface } \\
\text { Feature }\end{array}$ & $\begin{array}{c}\text { Model } \\
\text { East }\end{array}$ & $\begin{array}{l}\text { Model } \\
\text { North }\end{array}$ & $\begin{array}{l}\text { Model } \\
\text { Depth }\end{array}$ & $\begin{array}{l}\text { Strike } \\
\text { Direct }\end{array}$ & $\begin{array}{l}\text { Dip } \\
\text { Direct }\end{array}$ & Dip & Comments \\
\hline & & 2.53 & 0.91 & -3.96 & $\mathrm{~N}-\mathrm{S}$ & & & subvertical tight fracture \\
\hline \multicolumn{9}{|l|}{ WT1? } \\
\hline & & 0.79 & 1.68 & -0.76 & N20E & SE & 80 & $\begin{array}{l}\text { major open subvertical } \\
\text { fracture }\end{array}$ \\
\hline & & 1.14 & 1.68 & -0.76 & & & 90 & $\begin{array}{l}\text { minor tight vertical } \\
\text { fracture }\end{array}$ \\
\hline & & 1.85 & 1.68 & -0.76 & & & 90 & $\begin{array}{l}\text { moderate open wet vertical } \\
\text { fracture }\end{array}$ \\
\hline & & 1.88 & 1.68 & -0.76 & & & & $\begin{array}{l}\text { minor tight subvertical } \\
\text { fracture }\end{array}$ \\
\hline & & 2.08 & 1.68 & -0.76 & & & & $\begin{array}{l}\text { moderate sharp subvertical } \\
\text { open wet fracture }\end{array}$ \\
\hline \multicolumn{9}{|l|}{ WT2 } \\
\hline & LBT2 & 0.23 & 1.68 & -1.68 & $\sim \mathrm{N}-\mathrm{S}$ & $E$ & 80 & $\begin{array}{l}\text { subvertical angled } \\
\text { moderate, partly open } \\
\text { fracture }\end{array}$ \\
\hline & LBT2 & 0.25 & 1.68 & -1.68 & $\sim \mathrm{N}-\mathrm{S}$ & $E$ & 80 & $\begin{array}{l}\text { subvertical angled } \\
\text { moderate, partly open } \\
\text { fracture }\end{array}$ \\
\hline & & 0.51 & 1.68 & -1.68 & & & 90 & $\begin{array}{l}\text { vertical, moderate-major } \\
\text { fracture }\end{array}$ \\
\hline & & 1.04 & 1.68 & -1.68 & N60E & SE & 80 & $\begin{array}{l}\text { sharp angled minor- } \\
\text { moderate, open fracture }\end{array}$ \\
\hline & & 1.09 & 1.68 & -1.68 & N60E & SE & 80 & $\begin{array}{l}\text { sharp angled minor- } \\
\text { moderate, open fracture }\end{array}$ \\
\hline & & 1.57 & 1.68 & -1.68 & $\mathrm{~N}-\mathrm{S}$ & W & & $\begin{array}{l}\text { moderate subvertical open } \\
\text { fracture }\end{array}$ \\
\hline & LBT12(?) & 1.60 & 1.68 & -1.68 & $N-S$ & W & 90 & $\begin{array}{l}\text { moderate vertical open } \\
\text { fracture, but less open }\end{array}$ \\
\hline & & 1.70 & 1.68 & -1.68 & N20W & E & 80 & minor subvertical fracture \\
\hline & & 1.85 & 1.68 & -1.68 & & & 90 & $\begin{array}{l}\text { minor tight vertical } \\
\text { fracture }\end{array}$ \\
\hline \multicolumn{9}{|l|}{ WT3 } \\
\hline & & 0.11 & 1.68 & -3.05 & & & 90 & top zone vertical fractures \\
\hline & & 0.20 & 1.68 & -3.05 & & & 90 & base zone vertical fractures \\
\hline & LBT 2 & 0.66 & 1.68 & -3.05 & N20W & & 90 & open, wet vertical fracture \\
\hline & & 0.81 & 1.68 & -3.05 & N20W & E & & top tight subhorizontal \\
\hline & & 0.85 & 1.68 & -3.05 & N20W & $\mathrm{E}$ & & base tight subhorizontal \\
\hline & & 0.94 & 1.68 & -3.05 & N20W & W & 90 & $\begin{array}{l}\text { top partly open, vertical } \\
\text { fracture }\end{array}$ \\
\hline
\end{tabular}




\begin{tabular}{|c|c|c|c|c|c|c|c|c|}
\hline Hole & $\begin{array}{l}\text { Surface } \\
\text { Feature }\end{array}$ & $\begin{array}{c}\text { Model } \\
\text { East }\end{array}$ & $\begin{array}{l}\text { Model } \\
\text { North }\end{array}$ & $\begin{array}{l}\text { Model } \\
\text { Depth }\end{array}$ & $\begin{array}{l}\text { Strike } \\
\text { Direct }\end{array}$ & $\begin{array}{l}\text { Dip } \\
\text { Direct }\end{array}$ & Dip & Comments \\
\hline & & 0.99 & 1.68 & -3.05 & N20W & W & 90 & $\begin{array}{l}\text { base partly open, vertical } \\
\text { fracture }\end{array}$ \\
\hline & & 1.12 & 1.68 & -3.05 & N50W & $\mathrm{S}$ & 45 & moderate \\
\hline & & 1.27 & 1.68 & -3.05 & N50W & S & 45 & moderate \\
\hline & & 1.30 & 1.68 & -3.05 & N70E & $\mathrm{N}$ & 80 & $\begin{array}{l}\text { top fracture (cannot see } \\
\text { base) }\end{array}$ \\
\hline & & 1.33 & 1.68 & -3.05 & $\mathrm{~N}-\mathrm{S}$ & & 90 & vertical, wet, open fracture \\
\hline & LBT13 & 1.65 & 1.68 & -3.05 & N30W & W & & top subvertical fracture \\
\hline & LBT13 & 1.68 & 1.68 & -3.05 & $\mathrm{~N} 30 \mathrm{~W}$ & W & & base subvertical fracture \\
\hline & & 1.65 & 1.68 & -3.05 & $\mathrm{~N}-\mathrm{S}$ & $E$ & & top open, wet, subhorizontal \\
\hline & & 1.79 & 1.68 & -3.05 & $\mathrm{~N}-\mathrm{S}$ & E & & $\begin{array}{l}\text { base open, wet, } \\
\text { subhorizontal }\end{array}$ \\
\hline & & 2.25 & 1.68 & -3.05 & N10W & & 90 & fracture \\
\hline & & 2.25 & 1.68 & -3.05 & N60W & $\mathrm{S}$ & 70 & top open, wet fracture \\
\hline & & 2.36 & 1.68 & -3.05 & N60W & $S$ & 70 & base open, wet fracture \\
\hline & & 2.46 & 1.68 & -3.05 & $\mathrm{~N}-\mathrm{S}$ & & 90 & vertical fracture \\
\hline & & 2.51 & 1.68 & -3.05 & $\mathrm{~N}-\mathrm{S}$ & & 90 & tight, vertical fracture \\
\hline & & 2.59 & 1.68 & -3.05 & N10W & $\mathrm{S}$ & 80 & top tight fracture \\
\hline & & 2.51 & 1.68 & -3.05 & N10W & $S$ & 80 & base tight fracture \\
\hline & & 2.74 & 1.68 & -3.05 & & & 90 & open vertical, wet fracture \\
\hline \multicolumn{9}{|c|}{ UE25FRPTC\#1 } \\
\hline & LBT8 & 1.22 & 2.73 & -2.29 & & & subvert & $\begin{array}{l}\text { top major open/closed } \\
\text { fracture }\end{array}$ \\
\hline & LBT8 & 1.22 & 2.60 & -2.29 & & & subvert & $\begin{array}{l}\text { base major open/closed } \\
\text { fracture }\end{array}$ \\
\hline & & 1.22 & 2.57 & -2.29 & & & 90 & minor closed fracture \\
\hline & & 1.22 & 1.85 & -2.29 & & & & $\begin{array}{l}\text { major open void/clean } \\
\text { fracture }\end{array}$ \\
\hline & & 1.22 & 1.72 & -2.29 & & & & hole intersection fracture \\
\hline & & 1.22 & 1.26 & -2.29 & & & subhor & $\begin{array}{l}\text { top moderate-major closed } \\
\text { fracture }\end{array}$ \\
\hline & & 1.22 & 1.06 & -2.29 & & & subhor & $\begin{array}{l}\text { base moderate-major closed } \\
\text { fracture }\end{array}$ \\
\hline & LBT3 & 1.22 & 1.06 & -2.29 & & & 90 & $\begin{array}{l}\text { moderate-major } \\
\text { open/closed fracture }\end{array}$ \\
\hline & LBT33 & 1.22 & 0.93 & -2.29 & & & subhor & $\begin{array}{l}\text { top minor-moderate closed } \\
\text { fracture }\end{array}$ \\
\hline & LBT33 & 1.22 & 0.46 & -2.29 & & & subhor & $\begin{array}{l}\text { base minor-moderate closed } \\
\text { fracture }\end{array}$ \\
\hline
\end{tabular}




\begin{tabular}{|c|c|c|c|c|c|c|c|c|}
\hline Hole & $\begin{array}{l}\text { Surface } \\
\text { Feature }\end{array}$ & $\begin{array}{c}\text { Model } \\
\text { East }\end{array}$ & $\begin{array}{l}\text { Model } \\
\text { North }\end{array}$ & $\begin{array}{l}\text { Model } \\
\text { Depth }\end{array}$ & $\begin{array}{l}\text { Strike } \\
\text { Direct }\end{array}$ & $\begin{array}{l}\text { Dip } \\
\text { Direct }\end{array}$ & Dip & Comments \\
\hline & LBT2 & 1.22 & 0.67 & -2.29 & & & 90 & $\begin{array}{l}\text { top moderate closed } \\
\text { fracture }\end{array}$ \\
\hline & LBT2 & 1.22 & 0.54 & -2.29 & & & 90 & $\begin{array}{l}\text { base moderate closed } \\
\text { fracture }\end{array}$ \\
\hline & & 1.22 & 0.21 & -2.29 & & & 90 & $\begin{array}{l}\text { top minor-moderate closed } \\
\text { fracture }\end{array}$ \\
\hline & & 1.22 & 0.08 & -2.29 & & & 90 & $\begin{array}{l}\text { base minor-moderate closed } \\
\text { fracture }\end{array}$ \\
\hline \multicolumn{9}{|c|}{ UE25FRPTC\#2 } \\
\hline & & 1.22 & 3.03 & -2.01 & & & $\sim 90$ & top major open fracture \\
\hline & & 1.22 & 2.91 & -1.99 & & & $\sim 90$ & base major open fracture \\
\hline & & 1.22 & 2.78 & -1.97 & & & subhor & top minor closed fracture \\
\hline & & 1.22 & 2.60 & -1.94 & & & subhor & base minor closed fracture \\
\hline & & 1.22 & 2.48 & -1.92 & & & & pre-test borehole \\
\hline & & 1.22 & 1.95 & -1.82 & & & 90 & $\begin{array}{l}\text { minor-moderate closed } \\
\text { fracture }\end{array}$ \\
\hline & & 1.22 & 1.79 & -1.80 & & & & pre-test borehole \\
\hline & & 1.22 & 1.24 & -1.70 & & & & pre-test borehole \\
\hline & & 1.22 & 1.04 & -1.66 & & & 90 & moderate open fracture \\
\hline & & 1.22 & 0.86 & -1.63 & & & subhor & top major open fracture \\
\hline & & 1.22 & 0.56 & -1.58 & & & subhor & base major open fracture \\
\hline & & 1.22 & 0.71 & -1.60 & & & $\sim 45$ & $\begin{array}{l}\text { top moderate closed } \\
\text { fracture }\end{array}$ \\
\hline & & 1.22 & 0.45 & -1.56 & & & $\sim 45$ & $\begin{array}{l}\text { base moderate closed } \\
\text { fracture }\end{array}$ \\
\hline & & 1.22 & 0.58 & -1.58 & & & & pre-test borehole \\
\hline & LBT2 & 1.22 & 0.43 & -1.56 & & & $\sim 45$ & $\begin{array}{l}\text { top moderate-major } \\
\text { closed/open fracture }\end{array}$ \\
\hline & LBT2 & 1.22 & 0.23 & -1.52 & & & $\sim 45$ & $\begin{array}{l}\text { base moderate-major } \\
\text { closed/open fracture }\end{array}$ \\
\hline \multicolumn{9}{|c|}{ UE25FRPTC\#3 } \\
\hline & & 1.22 & 2.99 & -2.56 & & & $\sim 90$ & $\begin{array}{l}\text { top moderate-major } \\
\text { open/closed fracture }\end{array}$ \\
\hline & & 1.22 & 2.64 & -2.63 & & & $\sim 90$ & $\begin{array}{l}\text { base moderate-major } \\
\text { open/closed fracture }\end{array}$ \\
\hline & & 1.22 & 2.49 & -2.65 & & & $\sim 90$ & $\begin{array}{l}\text { minor-moderate closed } \\
\text { fracture }\end{array}$ \\
\hline & & 1.22 & 2.47 & -2.66 & & & subvert & $\begin{array}{l}\text { top moderate open/closed } \\
\text { fracture }\end{array}$ \\
\hline
\end{tabular}




\begin{tabular}{|c|c|c|c|c|c|c|c|c|}
\hline Hole & $\begin{array}{l}\text { Surface } \\
\text { Feature }\end{array}$ & $\begin{array}{c}\text { Model } \\
\text { East }\end{array}$ & $\begin{array}{l}\text { Model } \\
\text { North }\end{array}$ & $\begin{array}{l}\text { Model } \\
\text { Depth }\end{array}$ & $\begin{array}{l}\text { Strike } \\
\text { Direct }\end{array}$ & $\begin{array}{l}\text { Dip } \\
\text { Direct }\end{array}$ & Dip & Comments \\
\hline & & 1.22 & 2.37 & -2.67 & & & subvert & $\begin{array}{l}\text { base moderate open/closed } \\
\text { fracture }\end{array}$ \\
\hline & & 1.22 & 2.29 & -2.69 & & & & post-test borehole \\
\hline & & 1.22 & 2.04 & -2.73 & & & & post-test borehole \\
\hline & & 1.22 & 1.92 & -2.75 & & & & post-test borehole \\
\hline & & 1.22 & 1.85 & -2.77 & & & & post-test borehole \\
\hline & & 1.22 & 2.02 & -2.74 & & & subvert & top minor closed fracture \\
\hline & & 1.22 & 1.87 & -2.76 & & & subvert & base minor closed fracture \\
\hline & & 1.22 & 1.97 & -2.74 & & & subhor & top major open fracture \\
\hline & & 1.22 & 1.82 & -2.77 & & & subhor & base major open fracture \\
\hline & & 1.22 & 1.85 & -2.77 & & & & $\begin{array}{l}\text { possible major open } \\
\text { subvertical fracture }\end{array}$ \\
\hline & & 1.22 & 1.75 & -2.78 & & & 90 & minor closed fracture \\
\hline & LBT23 & 1.22 & 1.75 & -2.78 & & & $\sim 45$ & $\begin{array}{l}\text { top minor open/closed } \\
\text { fracture }\end{array}$ \\
\hline & LBT23 & 1.22 & 1.55 & -2.82 & & & $\sim 45$ & $\begin{array}{l}\text { base minor open/closed } \\
\text { fracture }\end{array}$ \\
\hline & & 1.22 & 1.57 & -2.82 & & & & $\begin{array}{l}\text { horizontal borehole } \\
\text { intersection }\end{array}$ \\
\hline & & 1.22 & 1.25 & -2.87 & & & & $\begin{array}{l}\text { vertical borehole } \\
\text { intersection }\end{array}$ \\
\hline & & 1.22 & 1.50 & -2.83 & & & subvert & top minor closed fracture \\
\hline & & 1.22 & 1.20 & -2.88 & & & subvert & base minor closed fracture \\
\hline & & 1.22 & 1.27 & -2.87 & & & subvert & top minor closed fracture \\
\hline & & 1.22 & 1.12 & -2.89 & & & subvert & base minor closed fracture \\
\hline & LBT3 & 1.22 & 1.07 & -2.90 & & & 90 & $\begin{array}{l}\text { moderate open/closed } \\
\text { fracture }\end{array}$ \\
\hline & & 1.22 & 1.00 & -2.92 & & & subvert & top minor fracture \\
\hline & LBT2 & 1.22 & 0.92 & -2.93 & & & subvert & top moderate open fracture \\
\hline & LBT2 & 1.22 & 0.80 & -2.95 & & & subvert & base moderate open fracture \\
\hline & & 1.22 & 0.62 & -2.98 & & & & $\begin{array}{l}\text { vertical borehole } \\
\text { intersection }\end{array}$ \\
\hline & & 1.22 & 0.10 & -3.07 & & & subvert & $\begin{array}{l}\text { top major open/closed } \\
\text { fracture }\end{array}$ \\
\hline & & 1.22 & 0.00 & -3.09 & & & subvert & $\begin{array}{l}\text { base major open/closed } \\
\text { fracture }\end{array}$ \\
\hline
\end{tabular}




\begin{tabular}{|c|c|c|c|c|c|c|c|c|}
\hline Hole & $\begin{array}{l}\text { Surface } \\
\text { Feature }\end{array}$ & $\begin{array}{c}\text { Model } \\
\text { East }\end{array}$ & $\begin{array}{l}\text { Model } \\
\text { North }\end{array}$ & $\begin{array}{l}\text { Model } \\
\text { Depth }\end{array}$ & $\begin{array}{l}\text { Strike } \\
\text { Direct }\end{array}$ & $\begin{array}{l}\text { Dip } \\
\text { Direct }\end{array}$ & Dip & Comments \\
\hline \multicolumn{9}{|c|}{ UE25FRPTC\#4 } \\
\hline & & 1.22 & 2.44 & -1.51 & & & & pre-test borehole \\
\hline & & 1.22 & 2.04 & -1.37 & & & $\sim 90$ & $\begin{array}{l}\text { moderate open/closed } \\
\text { fracture }\end{array}$ \\
\hline & & 1.22 & 1.74 & -1.25 & & & & post-test borehole \\
\hline & & 1.22 & 1.64 & -1.22 & & & & post-test borehole \\
\hline & & 1.22 & 1.24 & -1.08 & & & $\sim 45$ & minor-moderate ? fracture \\
\hline & LBT3 & 1.22 & 0.94 & -0.96 & & & subhor & top major open fracture \\
\hline & LBT3 & 1.22 & 1.13 & -1.03 & & & subhor & base major open fracture \\
\hline & & 1.22 & 0.59 & -0.84 & & & & pre-test borehole \\
\hline & & 1.22 & 0.47 & -0.79 & & & angled & top major open fracture \\
\hline & & 1.22 & 0.26 & -0.72 & & & angled & base major open fracture \\
\hline \multicolumn{9}{|c|}{ UE25FRPTC\#5 } \\
\hline & & 1.22 & 2.96 & -2.87 & & & $\sim 90$ & major open fracture \\
\hline & & 1.22 & 2.60 & -3.00 & & & $\sim 90$ & major open fracture \\
\hline & LBT8 & 1.22 & 2.60 & -3.00 & & & 90 & $\begin{array}{l}\text { moderate open/closed } \\
\text { fracture }\end{array}$ \\
\hline & & 1.22 & 2.55 & -3.02 & & & & pre-test borehole \\
\hline & LBT19(?) & 1.22 & 2.55 & -3.02 & & & $\sim 80$ & top moderate open fracture \\
\hline & LBT19(?) & 1.22 & 2.34 & -3.10 & & & $\sim 80$ & base moderate open fracture \\
\hline & LBT8 & 1.22 & 2.58 & -3.01 & & & $\sim 80$ & $\begin{array}{l}\text { top moderate open/closed } \\
\text { fracture }\end{array}$ \\
\hline & LBT8 & 1.22 & 2.24 & -3.13 & & & $\sim 80$ & $\begin{array}{l}\text { base moderate open/closed } \\
\text { fracture }\end{array}$ \\
\hline & LBT12 & 1.22 & 2.10 & -3.19 & & & $\sim 90$ & $\begin{array}{l}\text { top minor-moderate closed } \\
\text { fracture }\end{array}$ \\
\hline & LBT12 & 1.22 & 2.02 & -3.21 & & & $\sim 90$ & $\begin{array}{l}\text { base minor-moderate closed } \\
\text { fracture }\end{array}$ \\
\hline & & 1.22 & 1.97 & -3.23 & & & & post-test borehole \\
\hline & & 1.22 & 1.90 & -3.26 & & & & post-test borehole \\
\hline & & 1.22 & 1.83 & -3.28 & & & $\sim 90$ & $\begin{array}{l}\text { top minor-moderate } \\
\text { open/closed fracture }\end{array}$ \\
\hline & & 1.22 & 1.78 & -3.30 & & & $\sim 90$ & $\begin{array}{l}\text { base minor-moderate } \\
\text { open/closed fracture }\end{array}$ \\
\hline & & 1.22 & 1.73 & -3.32 & & & $\sim 90$ & $\begin{array}{l}\text { top minor-moderate } \\
\text { open/closed fracture }\end{array}$ \\
\hline & & 1.22 & 1.71 & -3.33 & & & $\sim 90$ & $\begin{array}{l}\text { base minor-moderate } \\
\text { open/closed fracture }\end{array}$ \\
\hline & LBT12(?) & 1.22 & 1.69 & -3.34 & & & $\sim 80$ & top moderate open fracture \\
\hline
\end{tabular}




\begin{tabular}{|c|c|c|c|c|c|c|c|c|}
\hline Hole & $\begin{array}{l}\text { Surface } \\
\text { Feature }\end{array}$ & $\begin{array}{c}\text { Model } \\
\text { East }\end{array}$ & $\begin{array}{l}\text { Model } \\
\text { North }\end{array}$ & $\begin{array}{l}\text { Model } \\
\text { Depth }\end{array}$ & $\begin{array}{l}\text { Strike } \\
\text { Direct }\end{array}$ & $\begin{array}{l}\text { Dip } \\
\text { Direct }\end{array}$ & Dip & Comments \\
\hline & LBT12(?) & 1.22 & 1.47 & -3.42 & & & $\sim 80$ & base moderate open fracture \\
\hline & LBT12(?) & 1.22 & 1.61 & -3.36 & & & $\sim 80$ & top major open fracture \\
\hline & LBT12(?) & 1.22 & 1.18 & -3.52 & & & $\sim 80$ & base major open fracture \\
\hline & & 1.22 & 1.30 & -3.48 & & & & pre-test borehole \\
\hline & LBT2 & 1.22 & 1.18 & -3.52 & & & $\sim 70$ & $\begin{array}{l}\text { top moderate-major open } \\
\text { fracture }\end{array}$ \\
\hline & LBT2 & 1.22 & 1.11 & -3.55 & & & $\sim 70$ & $\begin{array}{l}\text { base moderate-major open } \\
\text { fracture }\end{array}$ \\
\hline & & 1.22 & 1.04 & -3.57 & & & $\sim 90$ & $\begin{array}{l}\text { moderate open/closed } \\
\text { fracture }\end{array}$ \\
\hline & & 1.22 & 0.65 & -3.71 & & & & pre-test borehole \\
\hline & & 1.22 & 0.63 & -3.72 & & & subhor & $\begin{array}{l}\text { top major open/closed } \\
\text { fracture }\end{array}$ \\
\hline & & 1.22 & 0.29 & -3.84 & & & subhor & $\begin{array}{l}\text { base major open/closed } \\
\text { fracture }\end{array}$ \\
\hline & LBT15 & 1.22 & 0.48 & -3.77 & & & $\sim 80$ & top minor closed fracture \\
\hline & LBT15 & 1.22 & 0.36 & -3.82 & & & $\sim 80$ & base minor closed fracture \\
\hline & & 1.22 & 0.05 & -3.93 & & & & $\begin{array}{l}\text { possible breakout or } \\
\text { fracture }\end{array}$ \\
\hline \multicolumn{9}{|c|}{ UE25FRPTC\#6 } \\
\hline & & 1.22 & 3.04 & -1.40 & & & subvert & $\begin{array}{l}\text { top minor/tight closed } \\
\text { fracture }\end{array}$ \\
\hline & & 1.22 & 2.67 & -1.19 & & & subvert & $\begin{array}{l}\text { base minor/tight closed } \\
\text { fracture }\end{array}$ \\
\hline & & 1.22 & 2.44 & -1.05 & & & & pre-test borehole \\
\hline & & 1.22 & 1.88 & -0.73 & & & & pre-test borehole \\
\hline & LBT1 $(?)$ & 1.22 & 1.64 & -0.59 & & & & top major open fracture \\
\hline & LBT1 $(?)$ & 1.22 & 1.30 & -0.40 & & & & base major open fracture \\
\hline & & 1.22 & 1.25 & -0.37 & & & & pre-test borehole \\
\hline & LBT1(?) & 1.22 & 1.21 & -0.35 & & & subvert & $\begin{array}{l}\text { top minor-moderate } \\
\text { open/closed fracture }\end{array}$ \\
\hline & LBT1(?) & 1.22 & 1.17 & -0.32 & & & subvert & $\begin{array}{l}\text { base minor-moderate } \\
\text { open/closed fracture }\end{array}$ \\
\hline & LBT3 & 1.22 & 1.15 & -0.31 & & & subvert & $\begin{array}{l}\text { top minor-moderate } \\
\text { fracture }\end{array}$ \\
\hline & & 1.22 & 1.10 & -0.29 & & & subvert & $\begin{array}{l}\text { base minor-moderate } \\
\text { fracture }\end{array}$ \\
\hline & & 1.22 & 1.08 & -0.27 & & & $\sim 90$ & minor closed fracture \\
\hline & & 1.22 & 0.87 & -0.15 & & & $\sim 90$ & minor closed fracture \\
\hline
\end{tabular}




\begin{tabular}{|c|c|c|c|c|c|c|c|c|}
\hline Hole & $\begin{array}{l}\text { Surface } \\
\text { Feature }\end{array}$ & $\begin{array}{l}\text { Model } \\
\text { East }\end{array}$ & $\begin{array}{l}\text { Model } \\
\text { North }\end{array}$ & $\begin{array}{l}\text { Model } \\
\text { Depth }\end{array}$ & $\begin{array}{l}\text { Strike } \\
\text { Direct }\end{array}$ & $\begin{array}{l}\text { Dip } \\
\text { Direct }\end{array}$ & Dip & Comments \\
\hline \multicolumn{2}{|c|}{ UE25FRPTC\#7 } & \multicolumn{7}{|c|}{ very dirty hole with mudcake on the walls } \\
\hline & & 1.22 & 2.75 & -4.05 & & & & pre-test borehole \\
\hline & & 1.22 & 2.55 & -4.24 & & & & pre-test borehole \\
\hline & LBT8 & 1.22 & 2.18 & -4.59 & & & $\sim 60$ & top minor closed fracture \\
\hline & LBT8 & 1.22 & 2.09 & -4.68 & & & $\sim 60$ & base minor closed fracture \\
\hline & & 1.22 & 1.91 & -4.86 & & & & pre-test borehole \\
\hline & & 1.22 & 1.89 & -4.87 & & & $\sim 60$ & top minor closed fracture \\
\hline & & 1.22 & 1.82 & -4.94 & & & $\sim 60$ & base minor closed fracture \\
\hline & & 1.22 & 1.78 & -4.98 & & & $\sim 60$ & top minor closed fracture \\
\hline & & 1.22 & 1.71 & -5.05 & & & $\sim 60$ & base minor closed fracture \\
\hline & & 1.22 & 1.44 & -5.31 & & & subhor & $\begin{array}{l}\text { top moderate closed } \\
\text { fracture }\end{array}$ \\
\hline & & 1.22 & 1.31 & -5.44 & & & subhor & $\begin{array}{l}\text { base moderate closed } \\
\text { fracture }\end{array}$ \\
\hline & & 1.22 & 1.00 & -5.74 & & & $\sim 60$ & top minor closed fracture \\
\hline & & 1.22 & 0.96 & -5.77 & & & $\sim 60$ & base minor closed fracture \\
\hline & & 1.22 & 0.91 & -5.82 & & & subvert & top minor closed fracture \\
\hline & & 1.22 & 0.69 & -6.03 & & & subvert & base minor closed fracture \\
\hline & & 1.22 & 0.71 & -6.02 & & & subvert & major open fracture \\
\hline & & 1.22 & 0.44 & -6.28 & & & subvert & major open fracture \\
\hline \multicolumn{9}{|c|}{ UE25FRPTC\#8 } \\
\hline & & 1.22 & 2.57 & -0.61 & & & $\sim 45$ & top major closed fracture \\
\hline & & 1.22 & 2.41 & -0.48 & & & $\sim 45$ & base major closed fracture \\
\hline & LBT1 & 1.22 & 2.45 & -0.51 & & & $\sim 45$ & $\begin{array}{l}\text { top major open/closed } \\
\text { fracture }\end{array}$ \\
\hline & LBT1 & 1.22 & 2.35 & -0.43 & & & $\sim 45$ & $\begin{array}{l}\text { base major open/closed } \\
\text { fracture }\end{array}$ \\
\hline & & 1.22 & 2.37 & -0.44 & & & & pre-test borehole \\
\hline & & 1.22 & 2.37 & -0.44 & & & 90 & minor-moderate fracture \\
\hline & LBT16(?) & 1.22 & 2.22 & -0.31 & & & & top minor closed fracture \\
\hline & LBT16(?) & 1.22 & 2.08 & -0.20 & & & & base minor closed fracture \\
\hline & & 1.22 & 2.24 & -0.33 & & & $\sim 80$ & $\begin{array}{l}\text { top minor-moderate closed } \\
\text { fracture }\end{array}$ \\
\hline & & 1.22 & 1.98 & -0.11 & & & $\sim 80$ & $\begin{array}{l}\text { base minor-moderate closed } \\
\text { fracture }\end{array}$ \\
\hline \multicolumn{2}{|c|}{ UE25FRPTC\#9 } & \multicolumn{7}{|c|}{ locally muddy hole(covering tape) } \\
\hline & & 1.75 & 2.70 & -2.29 & & & 90 & $\begin{array}{l}\text { moderate open/closed } \\
\text { Discrete fracture }\end{array}$ \\
\hline
\end{tabular}




\begin{tabular}{|c|c|c|c|c|c|c|c|c|}
\hline Hole & $\begin{array}{l}\text { Surface } \\
\text { Feature }\end{array}$ & $\begin{array}{l}\text { Model } \\
\text { East }\end{array}$ & $\begin{array}{l}\text { Model } \\
\text { North }\end{array}$ & $\begin{array}{l}\text { Model } \\
\text { Depth }\end{array}$ & $\begin{array}{l}\text { Strike } \\
\text { Direct }\end{array}$ & $\begin{array}{l}\text { Dip } \\
\text { Direct }\end{array}$ & Dip & Comments \\
\hline & & 1.82 & 2.48 & -2.29 & & & & post-test borehole \\
\hline & LBT8 & 1.85 & 2.36 & -2.29 & & & $\sim 45$ & top minor closed fracture \\
\hline & LBT8 & 1.87 & 2.29 & -2.29 & & & $\sim 45$ & base minor closed fracture \\
\hline & & 1.87 & 2.29 & -2.29 & & & & top minor closed fracture \\
\hline & & 1.91 & 2.16 & -2.29 & & & & base minor closed fracture \\
\hline & & 1.99 & 1.89 & -2.29 & & & $\sim 90$ & minor closed fracture \\
\hline & & 2.02 & 1.77 & -2.29 & & & & post-test borehole \\
\hline & & 2.07 & 1.60 & -2.29 & & & & pre-test borehole \\
\hline & & 2.53 & 0.00 & -2.29 & & & $\sim 80$ & top minor closed fracture \\
\hline & & 2.16 & 1.28 & -2.29 & & & $\sim 80$ & base minor closed fracture \\
\hline & & 2.21 & 1.13 & -2.29 & & & $\sim 85$ & major open fracture \\
\hline & & 2.33 & 0.69 & -2.29 & & & $\sim 80$ & top minor closed fracture \\
\hline & & 2.36 & 0.59 & -2.29 & & & $\sim 80$ & base minor closed fracture \\
\hline \multicolumn{2}{|c|}{ UE25FRPTC\#10 } & \multicolumn{7}{|c|}{ difficult hole to log } \\
\hline & LBT1(?) & 0.88 & 1.83 & -0.41 & & & $\sim 90$ & top major open fracture \\
\hline & & 0.98 & 1.83 & -0.33 & & & $\sim 90$ & base major open fracture \\
\hline & & 1.15 & 1.83 & -0.20 & & & & pre-test borehole \\
\hline \multicolumn{2}{|c|}{ UE25FRPTC\#11 } & & & & & & & pre-test borehole \\
\hline & & 0.24 & 1.83 & -1.14 & & & $\sim 45$ & $\begin{array}{l}\text { top major open/closed } \\
\text { fracture }\end{array}$ \\
\hline & & 0.33 & 1.83 & -1.08 & & & $\sim 45$ & $\begin{array}{l}\text { base major open/closed } \\
\text { fracture }\end{array}$ \\
\hline & LBT16(?) & 1.05 & 1.83 & -0.62 & & & $\sim 45$ & $\begin{array}{l}\text { top minor-moderate closed } \\
\text { fracture }\end{array}$ \\
\hline & LBT16(?) & 1.14 & 1.83 & -0.55 & & & $\sim 45$ & $\begin{array}{l}\text { base minor-moderate closed } \\
\text { fracture }\end{array}$ \\
\hline & & 1.55 & 1.83 & -0.29 & & & & pre-test borehole \\
\hline \multicolumn{9}{|c|}{ UE25FRPTC\#12 } \\
\hline & & 0.10 & 1.83 & -1.46 & & & subvert & top major closed fracture \\
\hline & & 0.14 & 1.83 & -1.44 & & & subvert & base major closed fracture \\
\hline & & 0.24 & 1.83 & -1.39 & & & $\sim 90$ & minor closed fracture \\
\hline & & 0.38 & 1.83 & -1.32 & & & & pre-test borehole \\
\hline & LBT20(?) & 0.43 & 1.83 & -1.29 & & & $\sim 45$ & $\begin{array}{l}\text { top moderate-major open } \\
\text { fracture }\end{array}$ \\
\hline & LBT20(?) & 0.59 & 1.83 & -1.21 & & & $\sim 45$ & $\begin{array}{l}\text { base moderate-major open } \\
\text { fracture }\end{array}$ \\
\hline & & 1.06 & 1.83 & -0.97 & & & & pre-test borehole \\
\hline & LBT16(?) & 0.87 & 1.83 & -1.07 & & & $\sim 30$ & top minor closed fracture \\
\hline
\end{tabular}




\begin{tabular}{|c|c|c|c|c|c|c|c|c|}
\hline Hole & $\begin{array}{l}\text { Surface } \\
\text { Feature }\end{array}$ & $\begin{array}{l}\text { Model } \\
\text { East }\end{array}$ & $\begin{array}{l}\text { Model } \\
\text { North }\end{array}$ & $\begin{array}{l}\text { Model } \\
\text { Depth }\end{array}$ & $\begin{array}{l}\text { Strike } \\
\text { Direct }\end{array}$ & $\begin{array}{l}\text { Dip } \\
\text { Direct }\end{array}$ & Dip & Comments \\
\hline & LBT16(?) & 1.25 & 1.83 & -0.87 & & & $\sim 30$ & base minor closed fracture \\
\hline & & 1.32 & 1.83 & -0.84 & & & & post-test borehole \\
\hline & & 1.41 & 1.83 & -0.79 & & & & post-test borehole \\
\hline & LBT1 & 1.77 & 1.83 & -0.61 & & & subhor & top major open! fracture \\
\hline & LBT1 & 1.91 & 1.83 & -0.54 & & & subhor & base major open! fracture \\
\hline & & 1.98 & 1.83 & -0.50 & & & & pre-test borehole \\
\hline & & 2.28 & 1.83 & -0.35 & & & subhor & $\begin{array}{l}\text { top moderate closed } \\
\text { fracture }\end{array}$ \\
\hline & & 2.40 & 1.83 & -0.29 & & & subhor & $\begin{array}{l}\text { base moderate closed } \\
\text { fracture }\end{array}$ \\
\hline & & 2.45 & 1.83 & -0.26 & & & & $\begin{array}{l}\text { top moderate closed } \\
\text { fracture }\end{array}$ \\
\hline & & 2.61 & 1.83 & -0.18 & & & & $\begin{array}{l}\text { base moderate closed } \\
\text { fracture }\end{array}$ \\
\hline & & 2.59 & 1.83 & -0.19 & & & & pre-test borehole \\
\hline \multicolumn{9}{|c|}{ UE25FRPTC\#13 } \\
\hline & & -0.05 & 1.83 & -1.75 & & & subvert & $\begin{array}{l}\text { top minor-moderate } \\
\text { open/closed fracture }\end{array}$ \\
\hline & & 0.03 & 1.83 & -1.72 & & & subvert & $\begin{array}{l}\text { base minor-moderate } \\
\text { open/closed fracture }\end{array}$ \\
\hline & LBT20(?) & 0.45 & 1.83 & -1.57 & & & $\sim 90$ & minor-moderate fracture \\
\hline & & 1.03 & 1.83 & -1.35 & & & & post-test borehole \\
\hline & & 1.08 & 1.83 & -1.34 & & & & pre-test borehole \\
\hline & & 1.72 & 1.83 & -1.10 & & & & pre-test borehole \\
\hline & & 2.36 & 1.83 & -0.87 & & & & pre-test borehole \\
\hline & LBT1(?) & 2.53 & 1.83 & -0.81 & & & subhor & $\begin{array}{l}\text { top major open/closed } \\
\text { fracture }\end{array}$ \\
\hline & LBT1(?) & 2.39 & 1.83 & -0.86 & & & subhor & $\begin{array}{l}\text { base major open/closed } \\
\text { fracture }\end{array}$ \\
\hline \multicolumn{9}{|c|}{ UE25FRPTC\#14 } \\
\hline & LBT2 & 0.14 & 1.83 & -1.87 & & & $\sim 90$ & top major open fracture \\
\hline & LBT2 & 0.21 & 1.83 & -1.85 & & & $\sim 90$ & base major open fracture \\
\hline & & 1.22 & 1.83 & -1.60 & & & & pre-test borehole \\
\hline & LBT12 & 1.57 & 1.83 & -1.51 & & & $\sim 45$ & top major open fracture \\
\hline & LBT12 & 1.69 & 1.83 & -1.48 & & & $\sim 45$ & base major open fracture \\
\hline & & 1.77 & 1.83 & -1.47 & & & & pre-test borehole \\
\hline & LBT13 & 1.84 & 1.83 & -1.45 & & & $\sim 45$ & top minor closed fracture \\
\hline & LBT13 & 1.99 & 1.83 & -1.41 & & & $\sim 45$ & base minor closed fracture \\
\hline
\end{tabular}




\begin{tabular}{|c|c|c|c|c|c|c|c|c|}
\hline Hole & $\begin{array}{l}\text { Surface } \\
\text { Feature }\end{array}$ & $\begin{array}{c}\text { Model } \\
\text { East }\end{array}$ & $\begin{array}{l}\text { Model } \\
\text { North }\end{array}$ & $\begin{array}{l}\text { Model } \\
\text { Depth }\end{array}$ & $\begin{array}{l}\text { Strike } \\
\text { Direct }\end{array}$ & $\begin{array}{l}\text { Dip } \\
\text { Direct }\end{array}$ & Dip & Comments \\
\hline & & 2.43 & 1.83 & -1.30 & & & & pre-test borehole \\
\hline & & 2.51 & 1.83 & -1.28 & & & $\sim 45$ & $\begin{array}{l}\text { top minor-moderate } \\
\text { open/closed fracture }\end{array}$ \\
\hline & & 2.63 & 1.83 & -1.25 & & & $\sim 45$ & $\begin{array}{l}\text { base minor-moderate } \\
\text { open/closed fracture }\end{array}$ \\
\hline & & 2.60 & 1.83 & -1.26 & & & $\sim 45$ & $\begin{array}{l}\text { top minor-moderate } \\
\text { open/closed fracture }\end{array}$ \\
\hline & & 2.73 & 1.83 & -1.23 & & & $\sim 45$ & $\begin{array}{l}\text { base minor-moderate } \\
\text { open/closed fracture }\end{array}$ \\
\hline & & 2.73 & 1.83 & -1.23 & & & $\sim 60$ & $\begin{array}{l}\text { top minor-moderate } \\
\text { open/closed fracture }\end{array}$ \\
\hline & & 2.80 & 1.83 & -1.21 & & & $\sim 60$ & $\begin{array}{l}\text { base minor-moderate } \\
\text { open/closed fracture }\end{array}$ \\
\hline \multicolumn{9}{|c|}{ UE25FRPTC\#15 } \\
\hline & LBT20 & 0.16 & 1.83 & -2.26 & & & $\sim 90$ & top major open fracture \\
\hline & LBT20 & 0.51 & 1.83 & -2.25 & & & $\sim 90$ & base major open fracture \\
\hline & LBT2(?) & 0.35 & 1.83 & -2.25 & & & 90 & $\begin{array}{l}\text { top moderate closed } \\
\text { fracture }\end{array}$ \\
\hline & LBT2(?) & 0.93 & 1.83 & -2.24 & & & $\sim 80$ & $\begin{array}{l}\text { base moderate closed } \\
\text { fracture }\end{array}$ \\
\hline & & 1.12 & 1.83 & -2.24 & & & & \\
\hline & & 1.06 & 1.83 & -2.24 & & & subvert & $\begin{array}{l}\text { top moderate-major closed } \\
\text { fracture }\end{array}$ \\
\hline & & 1.57 & 1.83 & -2.23 & & & subvert & $\begin{array}{l}\text { base moderate-major closed } \\
\text { fracture }\end{array}$ \\
\hline & & 1.78 & 1.83 & -2.23 & & & & post-test borehole \\
\hline & & 1.91 & 1.83 & -2.23 & & & & post-test borehole \\
\hline & & 1.96 & 1.83 & -2.23 & & & subvert & $\begin{array}{l}\text { top moderate closed } \\
\text { fracture }\end{array}$ \\
\hline & & 2.41 & 1.83 & -2.22 & & & subvert & $\begin{array}{l}\text { base moderate closed } \\
\text { fracture }\end{array}$ \\
\hline & & 2.41 & 1.83 & -2.22 & & & & pre-test borehole \\
\hline \multicolumn{9}{|c|}{ UE25FRPTC\#16 } \\
\hline & LBT20(?) & 0.24 & 1.83 & -2.60 & & & $\sim 90$ & minor closed fracture \\
\hline & LBT2 & 0.37 & 1.83 & -2.62 & & & subvert & $\begin{array}{l}\text { top moderate open/closed } \\
\text { fracture }\end{array}$ \\
\hline & LBT2 & 0.45 & 1.83 & -2.63 & & & subvert & $\begin{array}{l}\text { base moderate open/closed } \\
\text { fracture }\end{array}$ \\
\hline & & 0.52 & 1.83 & -2.65 & & & & pre-test borehole \\
\hline & LBT23(?) & 0.57 & 1.83 & -2.66 & & & subvert & top moderate closed \\
\hline
\end{tabular}




\begin{tabular}{|c|c|c|c|c|c|c|c|c|}
\hline Hole & $\begin{array}{l}\text { Surface } \\
\text { Feature }\end{array}$ & $\begin{array}{c}\text { Model } \\
\text { East }\end{array}$ & $\begin{array}{l}\text { Model } \\
\text { North }\end{array}$ & $\begin{array}{l}\text { Model } \\
\text { Depth }\end{array}$ & $\begin{array}{l}\text { Strike } \\
\text { Direct }\end{array}$ & $\begin{array}{l}\text { Dip } \\
\text { Direct }\end{array}$ & Dip & Comments \\
\hline & & & & & & & & fracture \\
\hline & LBT23(?) & 0.93 & 1.83 & -2.72 & & & subvert & $\begin{array}{l}\text { base moderate closed } \\
\text { fracture }\end{array}$ \\
\hline & & 1.15 & 1.83 & -2.76 & & & & post-test borehole \\
\hline & & 1.28 & 1.83 & -2.78 & & & $\sim 90$ & $\begin{array}{l}\text { minor-moderate closed } \\
\text { fracture }\end{array}$ \\
\hline & & 1.58 & 1.83 & -2.83 & & & subvert & $\begin{array}{l}\text { top moderate open/closed } \\
\text { fracture }\end{array}$ \\
\hline & & 1.63 & 1.83 & -2.84 & & & subvert & $\begin{array}{l}\text { base moderate open/closed } \\
\text { fracture }\end{array}$ \\
\hline & & 1.73 & 1.83 & -2.86 & & & & post-test borehole \\
\hline & & 2.01 & 1.83 & -2.91 & & & $\sim 90$ & major open/closed fracture \\
\hline & & 2.39 & 1.83 & -2.98 & & & & pre-test borehole \\
\hline & & 2.19 & 1.83 & -2.94 & & & 90 & minor closed fracture \\
\hline & LBT8 & 2.74 & 1.83 & -3.04 & & & 90 & $\begin{array}{l}\text { minor-moderate } \\
\text { open/closed fracture }\end{array}$ \\
\hline \multicolumn{9}{|c|}{ UE25FRPTC\#17 } \\
\hline & & 0.06 & 1.83 & -2.86 & & & & $\begin{array}{l}\text { moderate-major open } \\
\text { fracture }\end{array}$ \\
\hline & & 0.39 & 1.83 & -2.98 & & & subvert & major open fracture \\
\hline & & 0.18 & 1.83 & -2.90 & & & $\sim 90$ & top major open! fracture \\
\hline & & 0.63 & 1.83 & -3.07 & & & $\sim 90$ & base major open! fracture \\
\hline & & 0.39 & 1.83 & -2.98 & & & subvert & $\begin{array}{l}\text { top moderate-major open } \\
\text { fracture }\end{array}$ \\
\hline & & 0.46 & 1.83 & -3.01 & & & subvert & $\begin{array}{l}\text { base moderate-major open } \\
\text { fracture }\end{array}$ \\
\hline & & 0.80 & 1.83 & -3.13 & & & subvert & $\begin{array}{l}\text { top moderate-major open } \\
\text { fracture }\end{array}$ \\
\hline & & 0.94 & 1.83 & -3.18 & & & subvert & $\begin{array}{l}\text { base moderate-major open } \\
\text { fracture }\end{array}$ \\
\hline & & 1.13 & 1.83 & -3.25 & & & & post-test borehole \\
\hline & & 1.28 & 1.83 & -3.31 & & & subvert & moderate-major fracture \\
\hline & LBT13 & 1.47 & 1.83 & -3.38 & & & subvert & major! open/closed fracture \\
\hline & LBT13 & 1.56 & 1.83 & -3.41 & & & subvert & major! open/closed fracture \\
\hline & & 1.68 & 1.83 & -3.45 & & & & post-test borehole \\
\hline & & 1.68 & 1.83 & -3.45 & & & subvert & $\begin{array}{l}\text { minor-moderate closed } \\
\text { fracture }\end{array}$ \\
\hline & & 2.02 & 1.83 & -3.58 & & & subvert & $\begin{array}{l}\text { minor-moderate closed } \\
\text { fracture }\end{array}$ \\
\hline & & 1.85 & 1.83 & -3.51 & & & subvert & minor closed fracture \\
\hline
\end{tabular}




\begin{tabular}{|c|c|c|c|c|c|c|c|c|}
\hline Hole & $\begin{array}{l}\text { Surface } \\
\text { Feature }\end{array}$ & $\begin{array}{c}\text { Model } \\
\text { East }\end{array}$ & $\begin{array}{l}\text { Model } \\
\text { North }\end{array}$ & $\begin{array}{l}\text { Model } \\
\text { Depth }\end{array}$ & $\begin{array}{l}\text { Strike } \\
\text { Direct }\end{array}$ & $\begin{array}{l}\text { Dip } \\
\text { Direct }\end{array}$ & Dip & Comments \\
\hline & & 1.90 & 1.83 & -3.53 & & & subvert & minor closed fracture \\
\hline & & 2.09 & 1.83 & -3.60 & & & subvert & moderate closed fracture \\
\hline & & 2.83 & 1.83 & -3.87 & & & subvert & moderate closed fracture \\
\hline & & 2.02 & 1.83 & -3.58 & & & & pre-test borehole \\
\hline & & 2.43 & 1.83 & -3.72 & & & subvert & moderate closed fracture \\
\hline & & 2.93 & 1.83 & -3.91 & & & subvert & moderate closed fracture \\
\hline & & 2.71 & 1.83 & -3.83 & & & & pre-test borehole \\
\hline \multicolumn{9}{|c|}{ UE25FRPTC\#18 } \\
\hline & & 0.45 & 1.83 & -3.67 & & & $\sim$ subver & minor fracture \\
\hline & LBT30(?) & 0.75 & 1.83 & -3.87 & & & subhor & $\begin{array}{l}\text { top moderate open/closed } \\
\text { fracture }\end{array}$ \\
\hline & LBT30(?) & 0.93 & 1.83 & -4.01 & & & subhor & $\begin{array}{l}\text { base moderate open/closed } \\
\text { fracture }\end{array}$ \\
\hline & & 0.85 & 1.83 & -3.95 & & & $\sim 50$ & top minor closed fracture \\
\hline & & 0.98 & 1.83 & -4.04 & & & $\sim 50$ & base minor closed fracture \\
\hline & LBT22(?) & 0.89 & 1.83 & -3.98 & & & subhor & top minor closed fracture \\
\hline & LBT22(?) & 1.00 & 1.83 & -4.05 & & & subhor & base minor closed fracture \\
\hline & & 1.37 & 1.83 & -4.31 & & & subvert & $\begin{array}{l}\text { top moderate closed } \\
\text { fracture }\end{array}$ \\
\hline & & 1.48 & 1.83 & -4.39 & & & subvert & $\begin{array}{l}\text { base moderate closed } \\
\text { fracture }\end{array}$ \\
\hline & & 1.52 & 1.83 & -4.42 & & & subvert & top moderate open fracture \\
\hline & & 1.56 & 1.83 & -4.45 & & & subvert & base moderate open fracture \\
\hline & & 1.62 & 1.83 & -4.49 & & & subvert & $\begin{array}{l}\text { top minor open/closed } \\
\text { fracture }\end{array}$ \\
\hline & & 1.65 & 1.83 & -4.51 & & & subvert & $\begin{array}{l}\text { base minor open/closed } \\
\text { fracture }\end{array}$ \\
\hline & & 1.73 & 1.83 & -4.56 & & & subvert & $\begin{array}{l}\text { top minor open/closed } \\
\text { fracture }\end{array}$ \\
\hline & & 1.81 & 1.83 & -4.62 & & & subvert & $\begin{array}{l}\text { base minor open/closed } \\
\text { fracture }\end{array}$ \\
\hline & & 2.00 & 1.83 & -4.75 & & & & post-test borehole \\
\hline & & 2.59 & 1.83 & -5.16 & & & & pre-test borehole \\
\hline \multicolumn{9}{|c|}{ UE25FRPTC\#19 } \\
\hline & & 0.27 & 1.83 & -2.16 & & & subvert & $\begin{array}{l}\text { top moderate-major } \\
\text { fracture }\end{array}$ \\
\hline & & 0.38 & 1.83 & -2.15 & & & subvert & $\begin{array}{l}\text { base moderate-major } \\
\text { fracture }\end{array}$ \\
\hline & & 0.45 & 1.83 & -2.15 & & & subvert & top minor fracture \\
\hline
\end{tabular}




\begin{tabular}{|c|l|l|l|l|l|l|l|l|}
\hline Hole & $\begin{array}{c}\text { Surface } \\
\text { Feature }\end{array}$ & $\begin{array}{c}\text { Model } \\
\text { East }\end{array}$ & $\begin{array}{c}\text { Model } \\
\text { North }\end{array}$ & $\begin{array}{c}\text { Model } \\
\text { Depth }\end{array}$ & $\begin{array}{c}\text { Strike } \\
\text { Direct }\end{array}$ & $\begin{array}{c}\text { Dip } \\
\text { Direct }\end{array}$ & Dip & \multicolumn{1}{|c|}{ Comments } \\
\hline & & 0.50 & 1.83 & -2.14 & & & subvert & base minor fracture \\
\hline & & 1.06 & 1.83 & -2.11 & & & subvert & top minor fracture \\
\hline & & 1.16 & 1.83 & -2.10 & & & subvert & base minor fracture \\
\hline & & 1.46 & 1.83 & -2.08 & & & 90 & major fracture \\
\hline & & 1.74 & 1.83 & -2.06 & & & & pre-test borehole \\
\hline & & 2.12 & 1.83 & -2.03 & & & & pre-test borehole \\
\hline & & 2.69 & 1.83 & -1.99 & & & $\sim 90$ & top major fracture \\
\hline & & 2.80 & 1.83 & -1.98 & & & $\sim 90$ & base major fracture \\
\hline & & & & & & & & \\
\hline & LBT20(?) & 0.54 & 1.24 & -2.14 & & & $\sim 90$ & top major open fracture \\
\hline & LBT20(?) & 0.64 & 1.21 & -2.13 & & & $\sim 90$ & base major open fracture \\
\hline & & 0.85 & 1.15 & -2.12 & & & & pre-test borehole \\
\hline & LBT3 & 1.00 & 1.10 & -2.11 & & & $\sim 90$ & top major open fracture \\
\hline & LBT3 & 1.23 & 1.04 & -2.09 & & & $\sim 90$ & base major open fracture \\
\hline & & 1.62 & 0.93 & -2.07 & & & $\sim 90$ & $\begin{array}{l}\text { moderate-major } \\
\text { open/closed fracture }\end{array}$ \\
\hline & & 2.01 & 0.82 & -2.04 & & & subhor & top moderate fracture \\
\hline & & 2.17 & 0.77 & -2.03 & & & subhor & base moderate fracture \\
\hline & & 2.12 & 0.79 & -2.03 & & & & pre-test borehole \\
\hline
\end{tabular}



Appendix B

Figures 



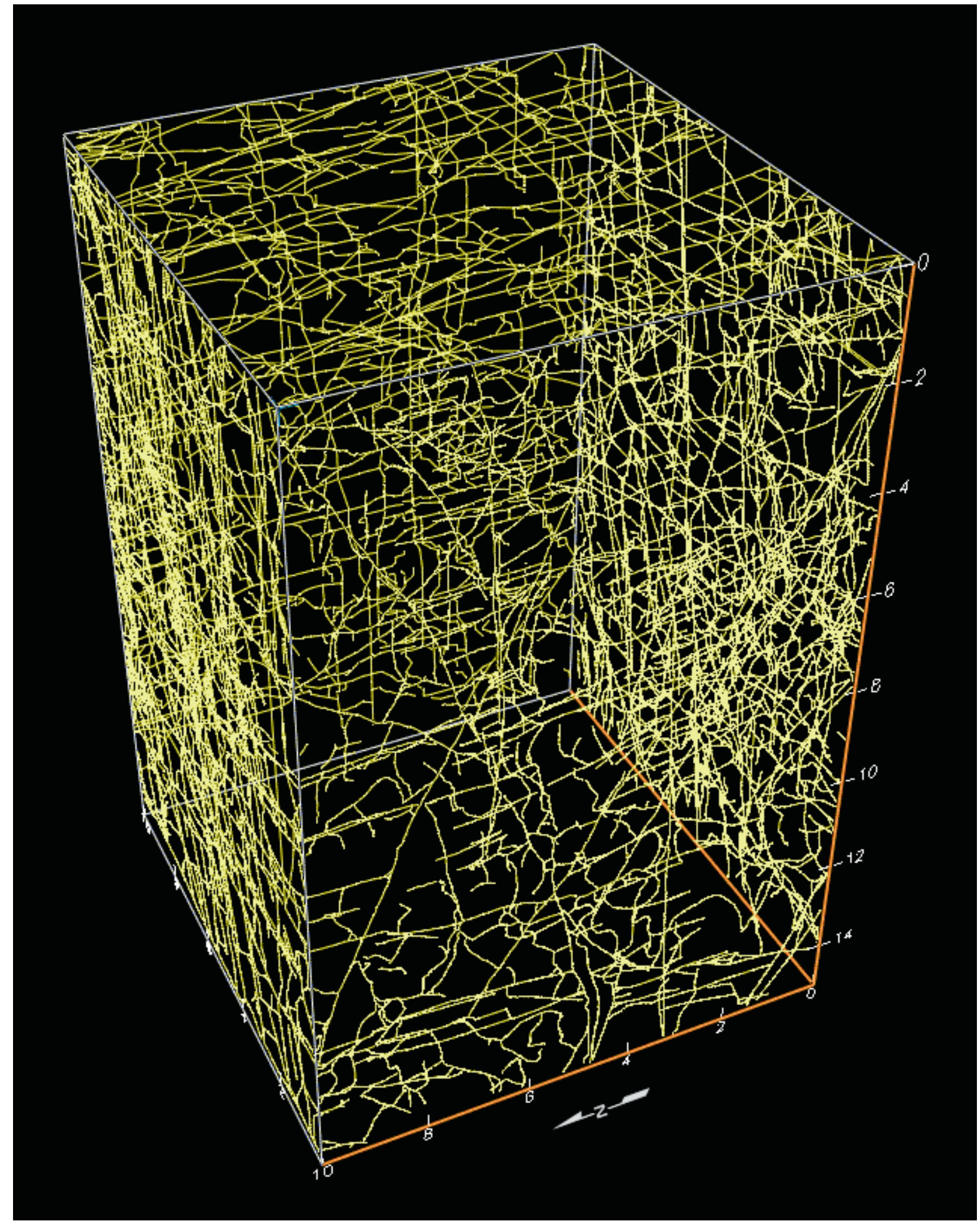

Figure 1. Mapped surface fractures on the large block: vertical and horizontal scales, in English units (ft), are equal and marked on the edges of the block. 

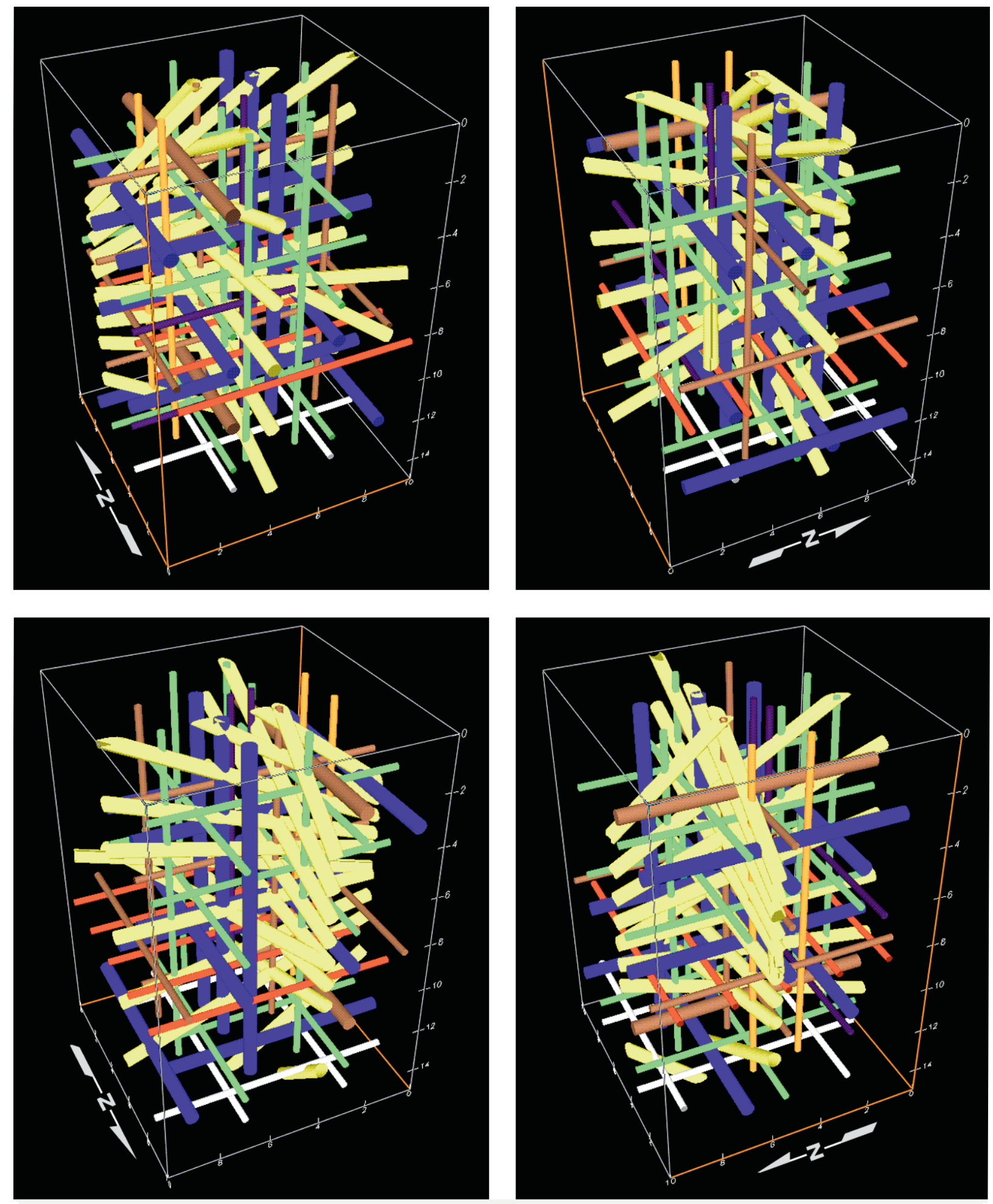

E9 E10 EH1 EH2 EH3 EH4 EH5 N1 N2 N3 NH1 NM1 NM2 NM3 WH1 WH2 WM1 WM2 WM3 $\square$ E03 NO1 NO2 WO5 $\square$ TE1 TR1 WR1 WR2 $\square$ E1 E7 NT1 NT2 NT3 NT4 WT1 WT2 WT3 E2 E3 E5 E6 E8 NN1 NN2 NN3 NN4 NN5 NN6 WN1 WN2 WN3 WN4 $\square$ Post-test holes

Figure 2. All boreholes in the large block: vertical and horizontal scales, in English units (ft), are equal and marked on the edges of the block. 


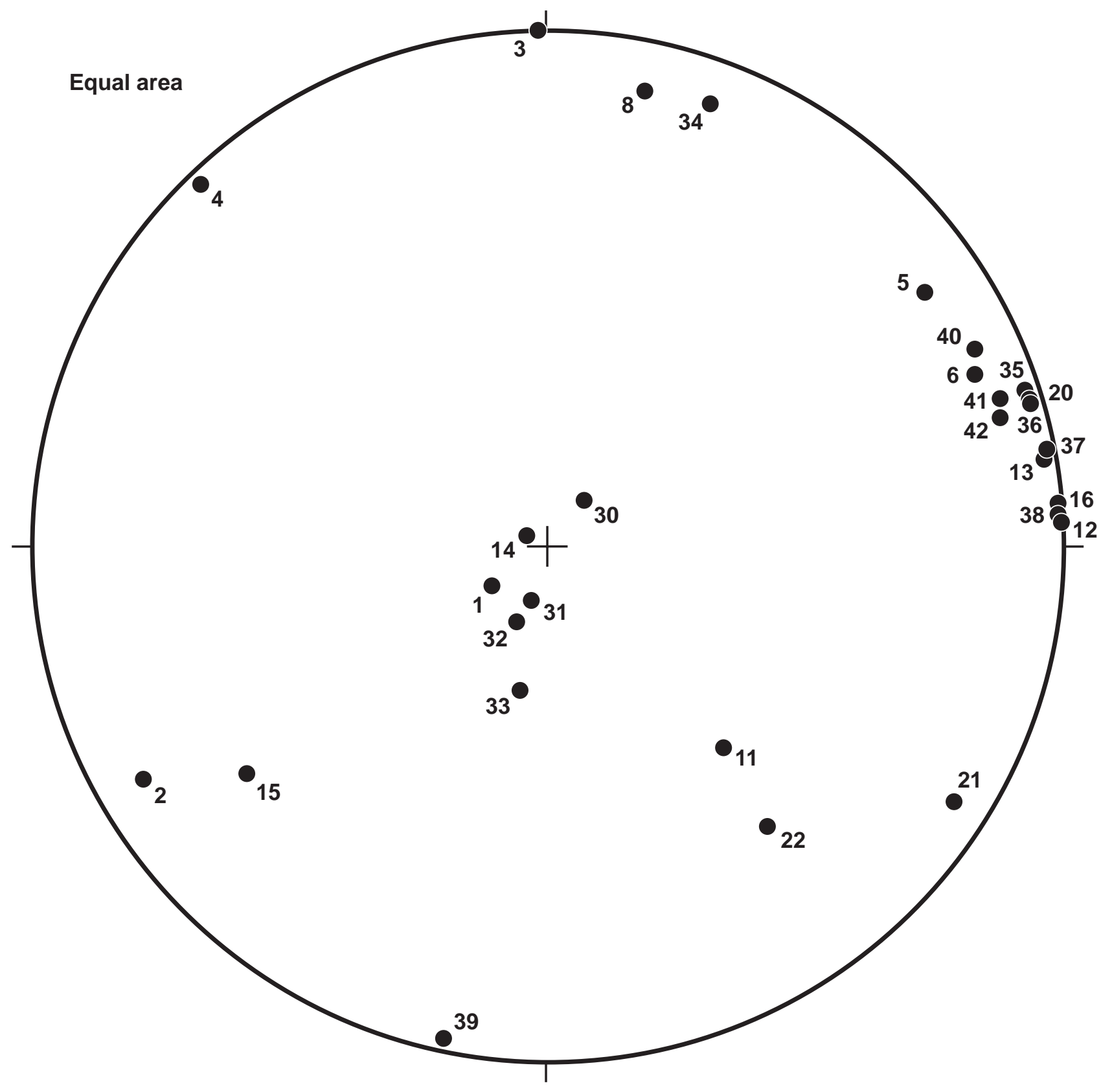

Figure 3. Equal-area diagram of poles to major fractures mapped for the LBT (Stereonet 4.9.5a by Richard Allmendinger of Cornell University in Ithaca, New York); fracture numbers correlate with fracture planes listed in Table 3 

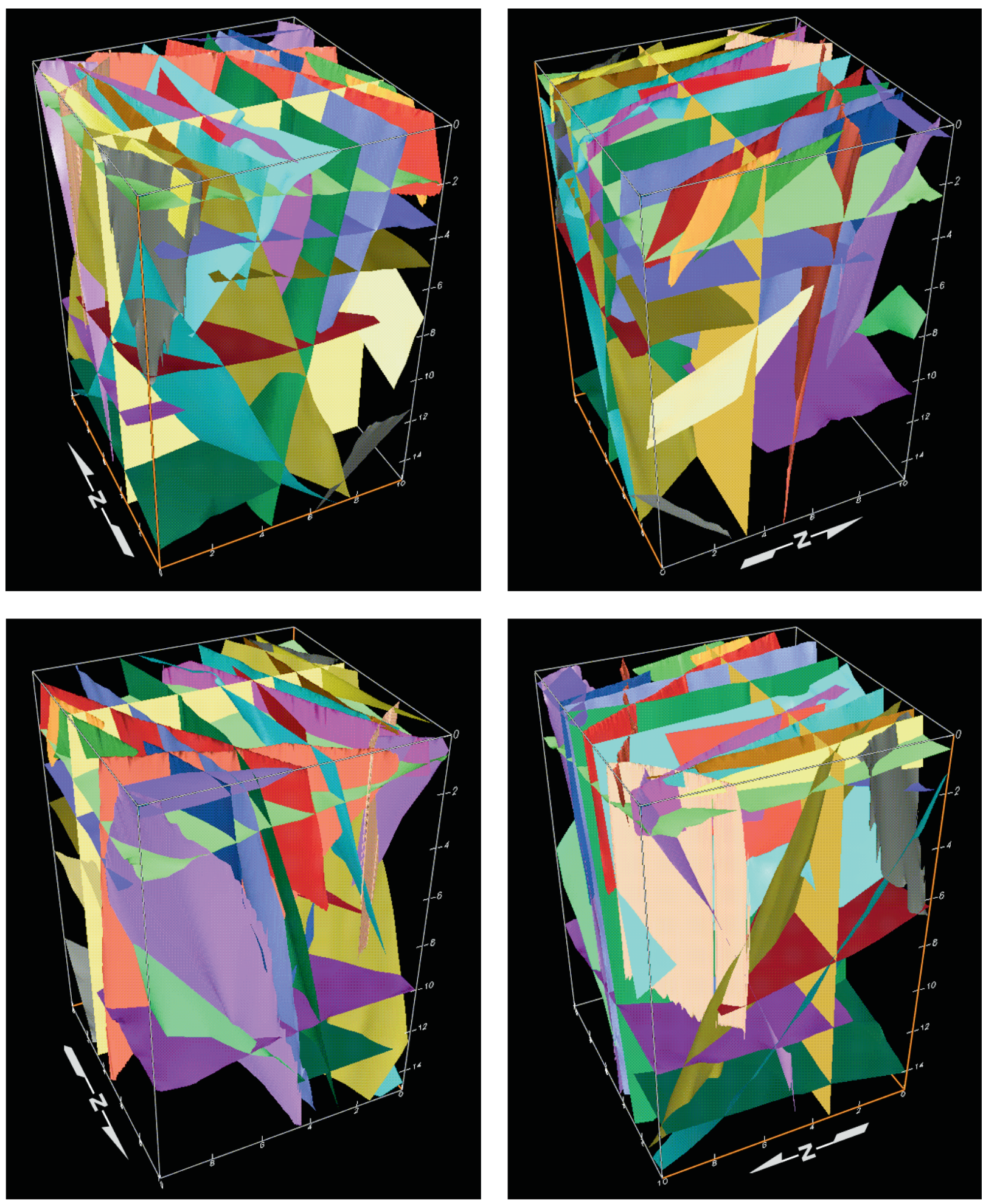

Figure 4. 3-D depiction of the major mappable fractures cutting the large block: individual fractures are color coded to facilitate discrimination of the fracture planes.; vertical and horizontal scales, in English units (ft), are equal and marked on the edges of the block. 

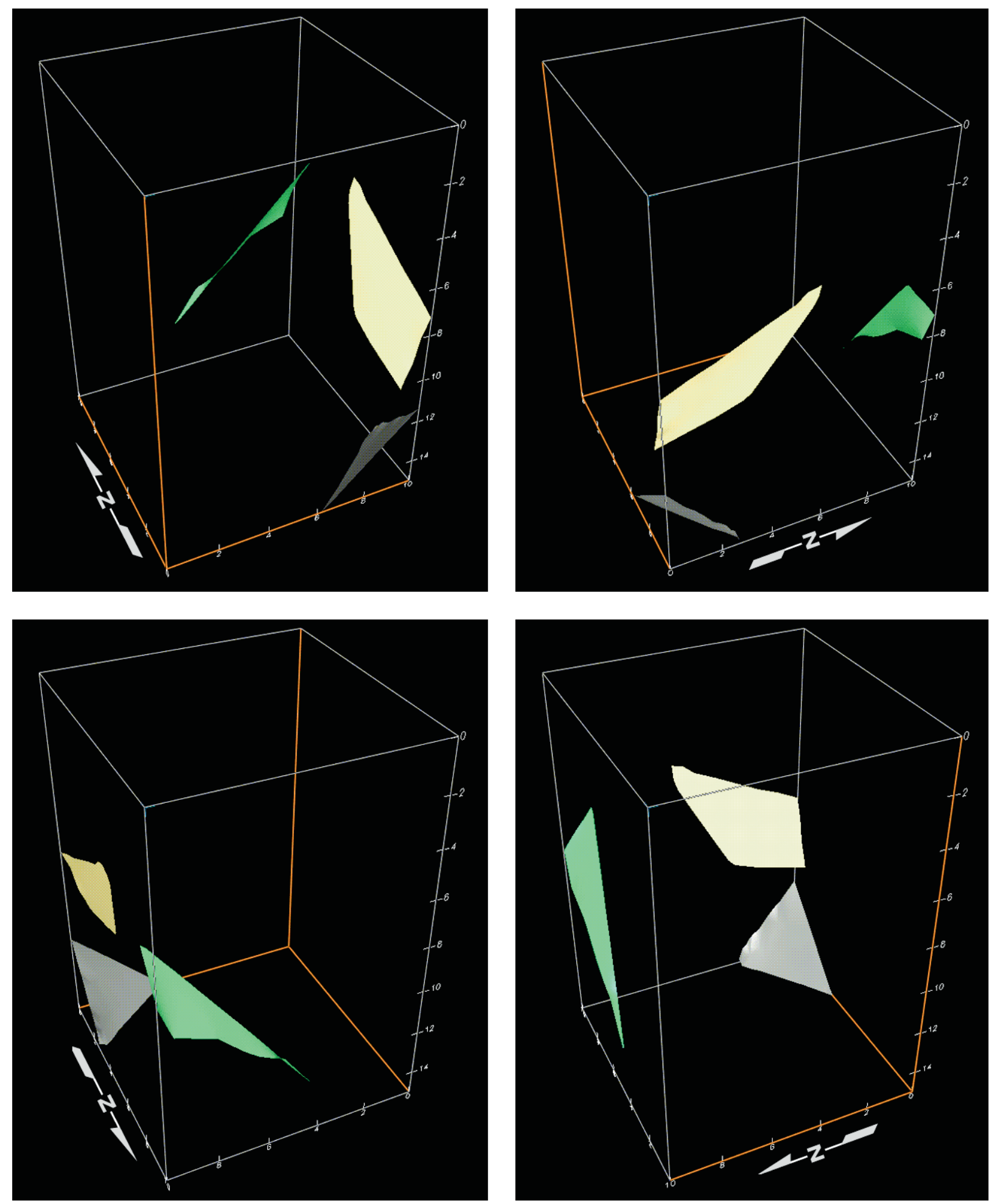

Figure 5. Fracture system \#1 contains 3 fractures that strike N50E and dip $40-45^{\circ}$ northwest: individual fractures are color coded to facilitate discrimination of the fracture planes; vertical and horizontal scales, in English units (ft), are equal and marked on the edges of the block. 

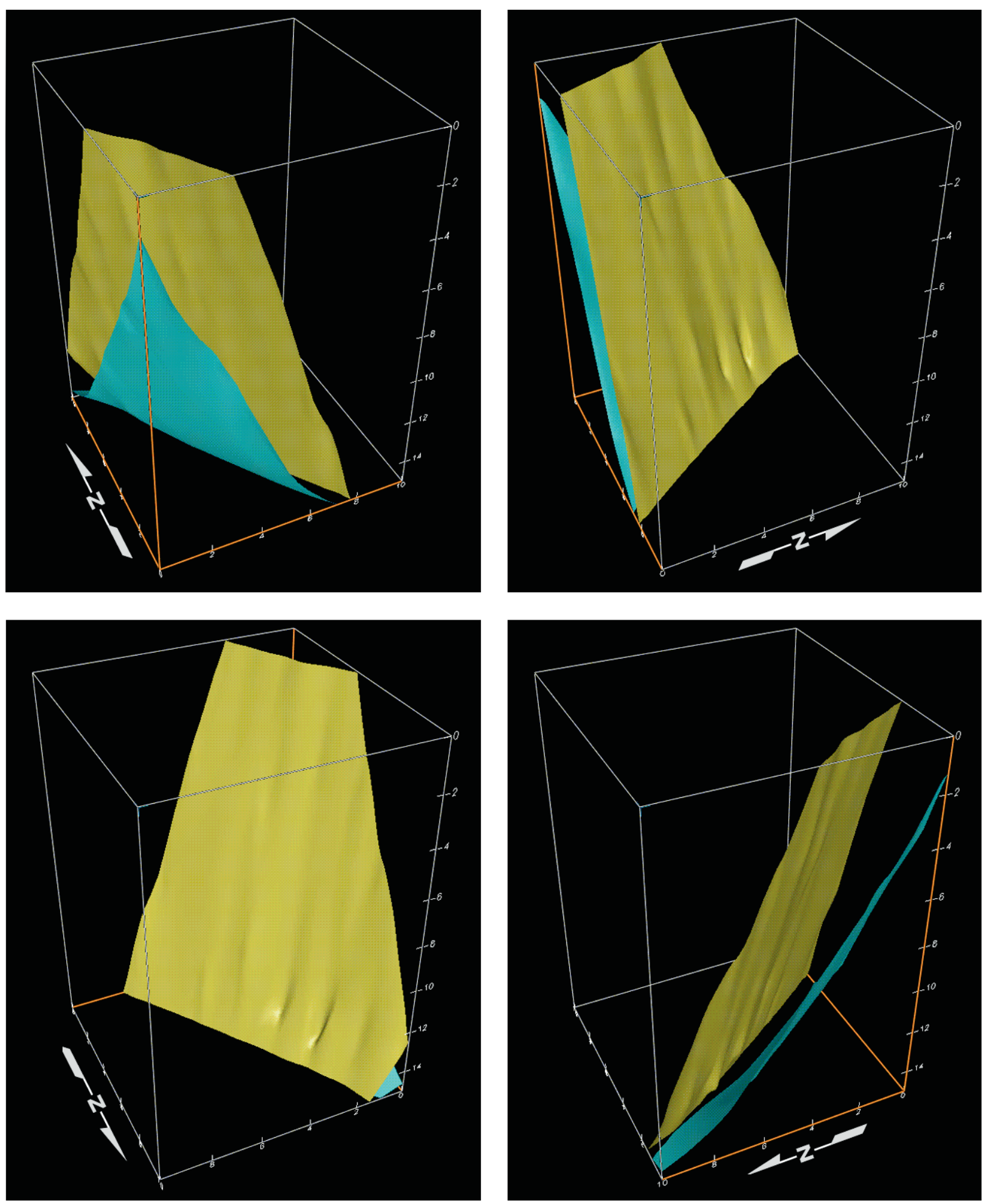

Figure 6. Fracture system \#2 contains 2 fractures that strike N30-40W and dip 60-80 northeast: individual fractures are color coded to facilitate discrimination of the fracture planes: vertical and horizontal scales, in English units (ft), are equal and marked on the edges of the block. 

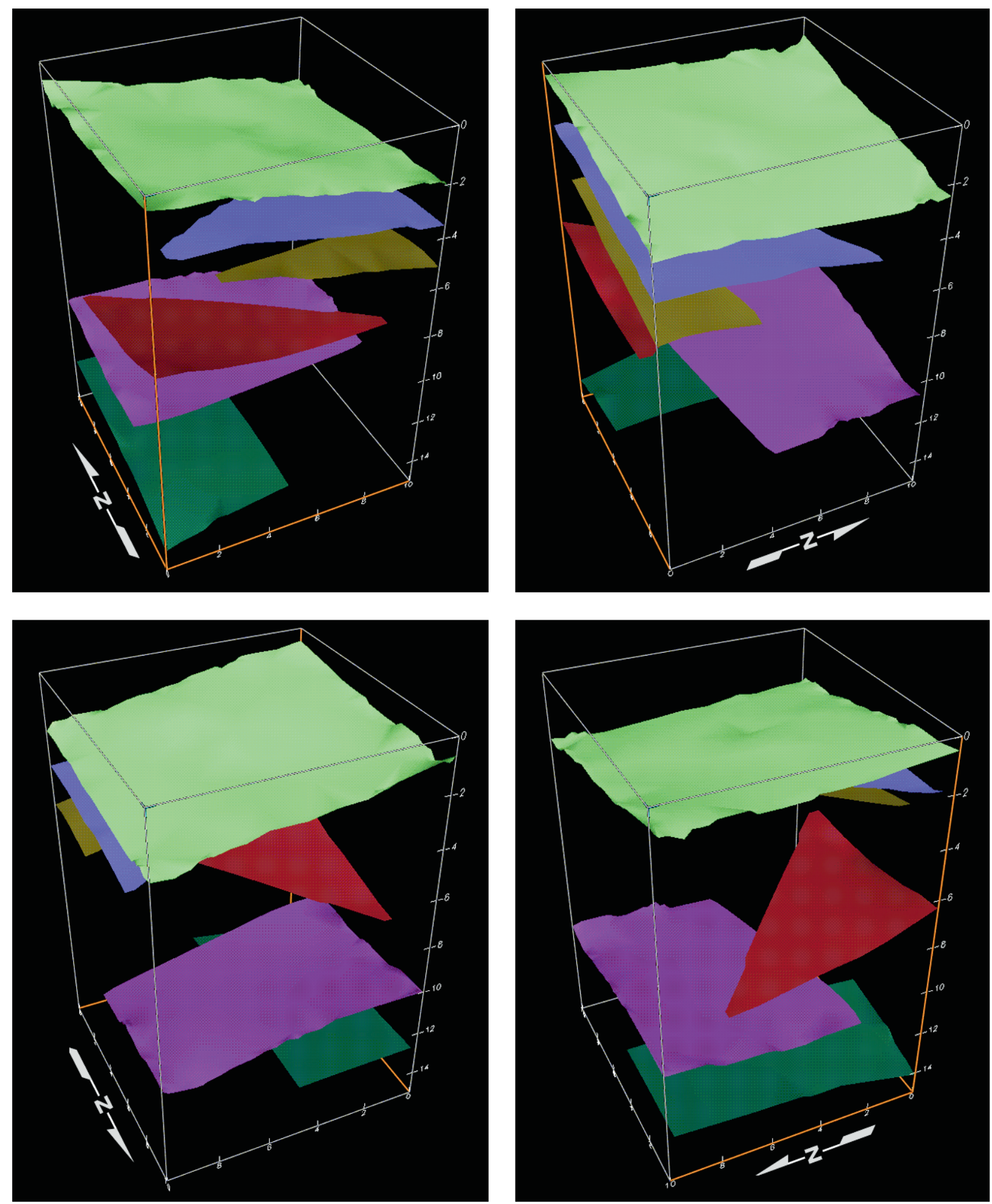

Figure 7. Fracture system \#3 contains 6 subhorizontal fractures. Fracture LBT1 (green) is the largest, most significant fracture mapped in the block. Individual fractures are color coded to facilitate discrimination of the fracture planes. The vertical and horizontal scales, in English units (ft), are equal and marked on the edges of the block. 

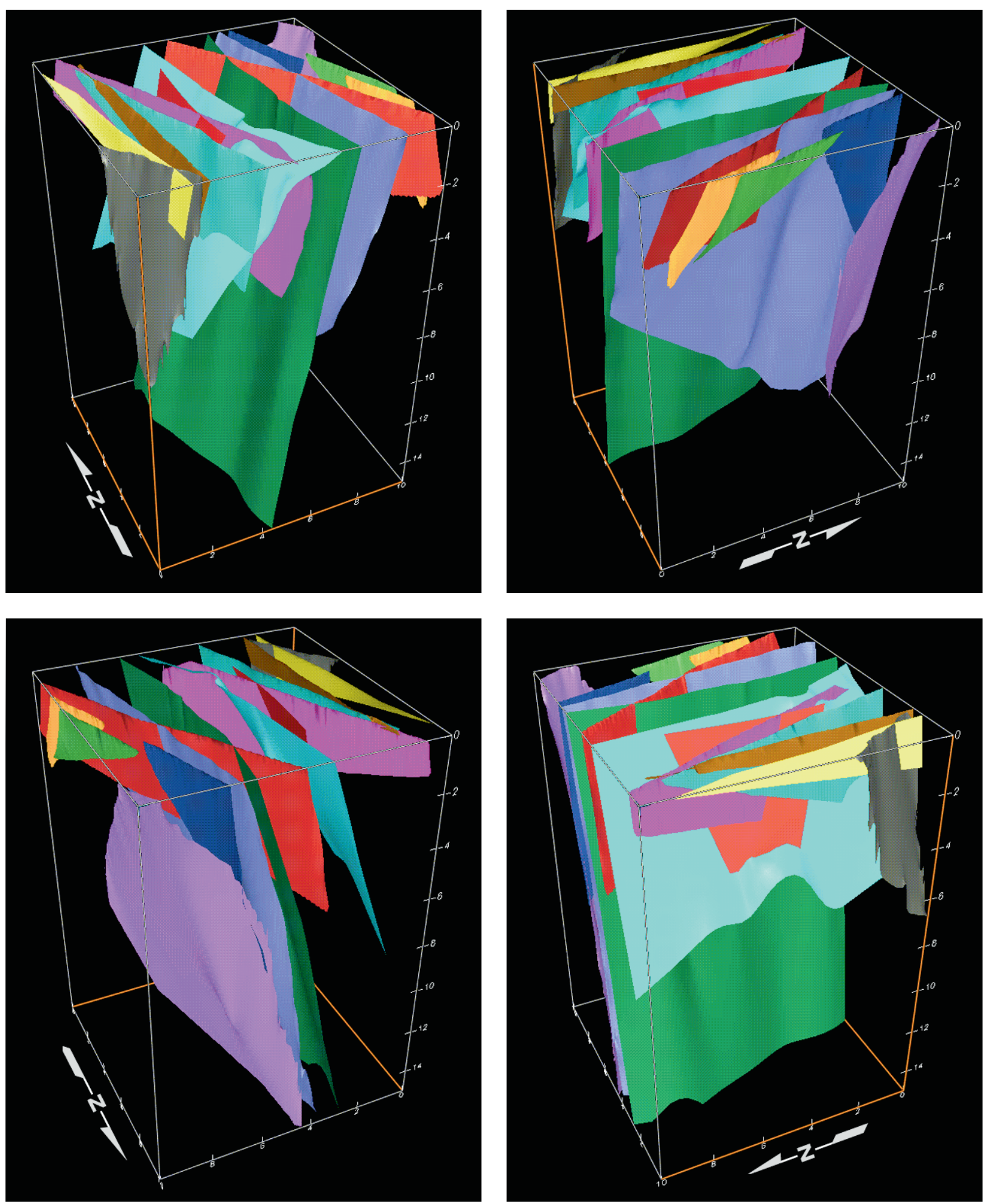

Figure 8. Fracture system \#4 contains 13 mapped fractures and is the dominant system in the block. This system strikes northwest and dips toward the southwest. Individual fractures are color coded to facilitate discrimination of the fracture planes. The vertical and horizontal scales, in English units (ft), are equal and marked on the edges of the block. 

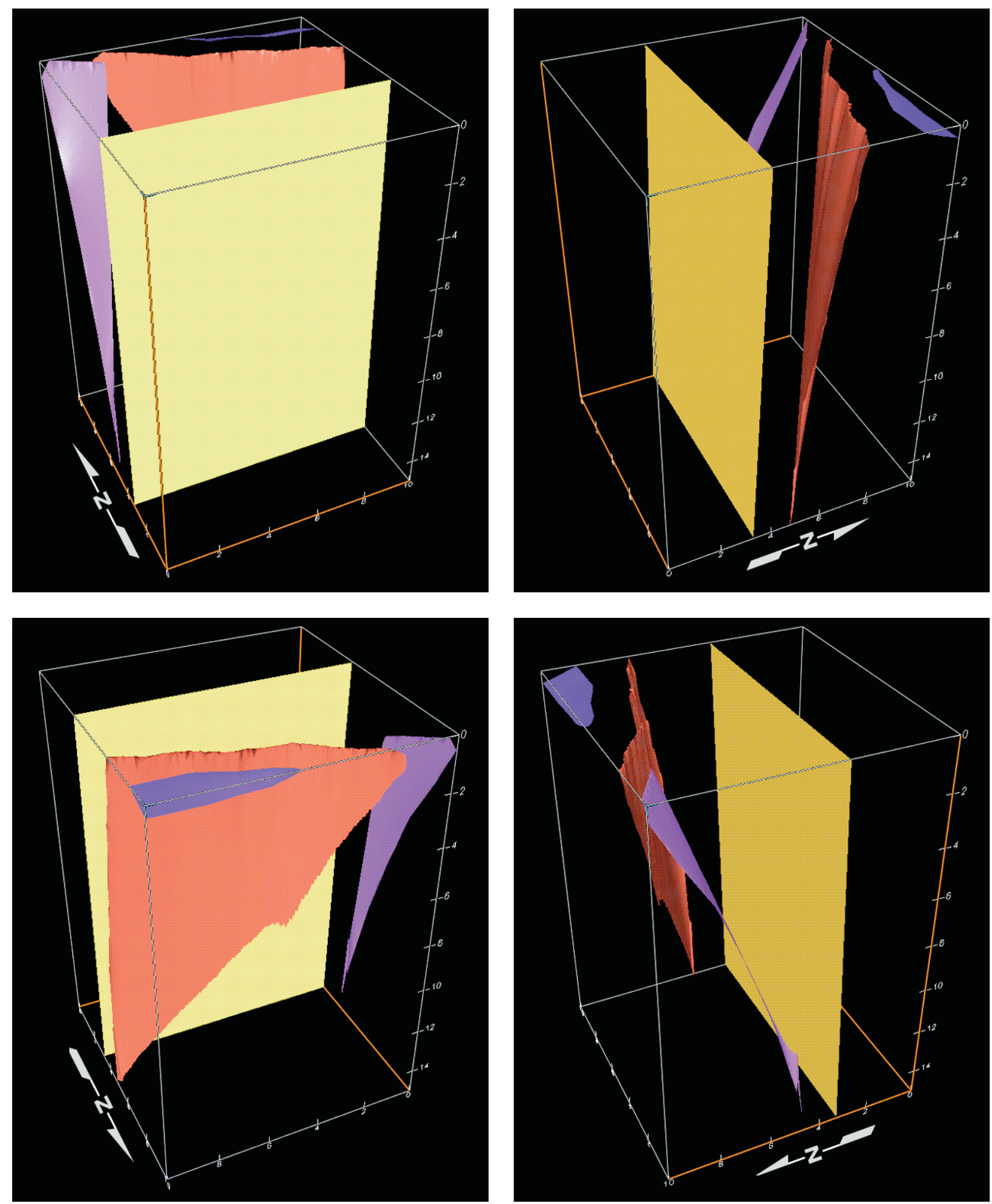

Figure 9. Fracture system \#5 contains 4 approximately vertical fractures that strike east-west. Individual fractures are color coded to facilitate discrimination of the fracture planes. The vertical and horizontal scales, in English units $(\mathrm{ft})$, are equal and marked on the edges of the block. 

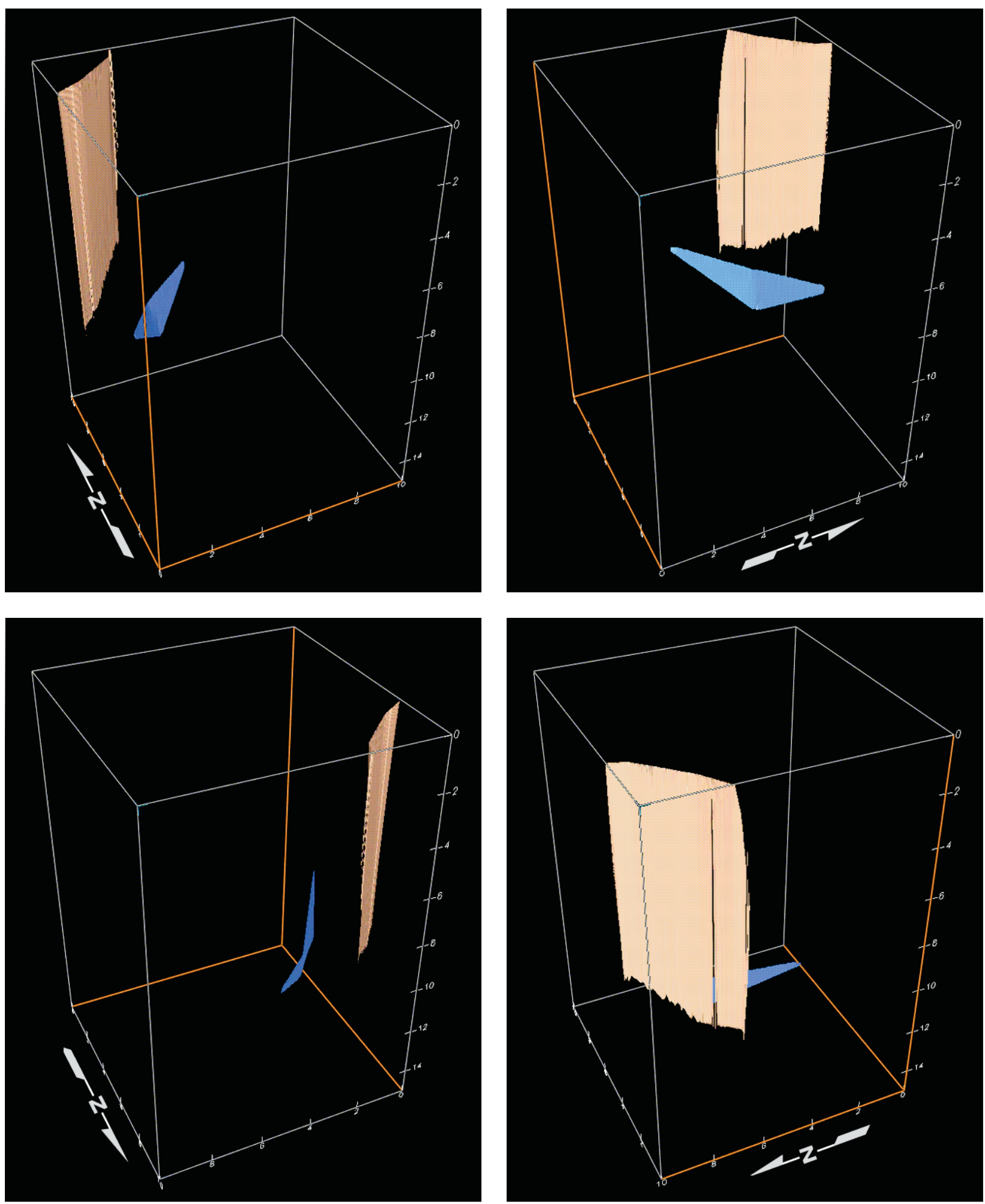

Figure 10. Fracture system \#6 contains 2 mappable fractures that strike northeast and dip to the southeast. Individual fractures are color coded to facilitate discrimination of the fracture planes. The vertical and horizontal scales, in English units (ft), are equal and marked on the edges of the block. 


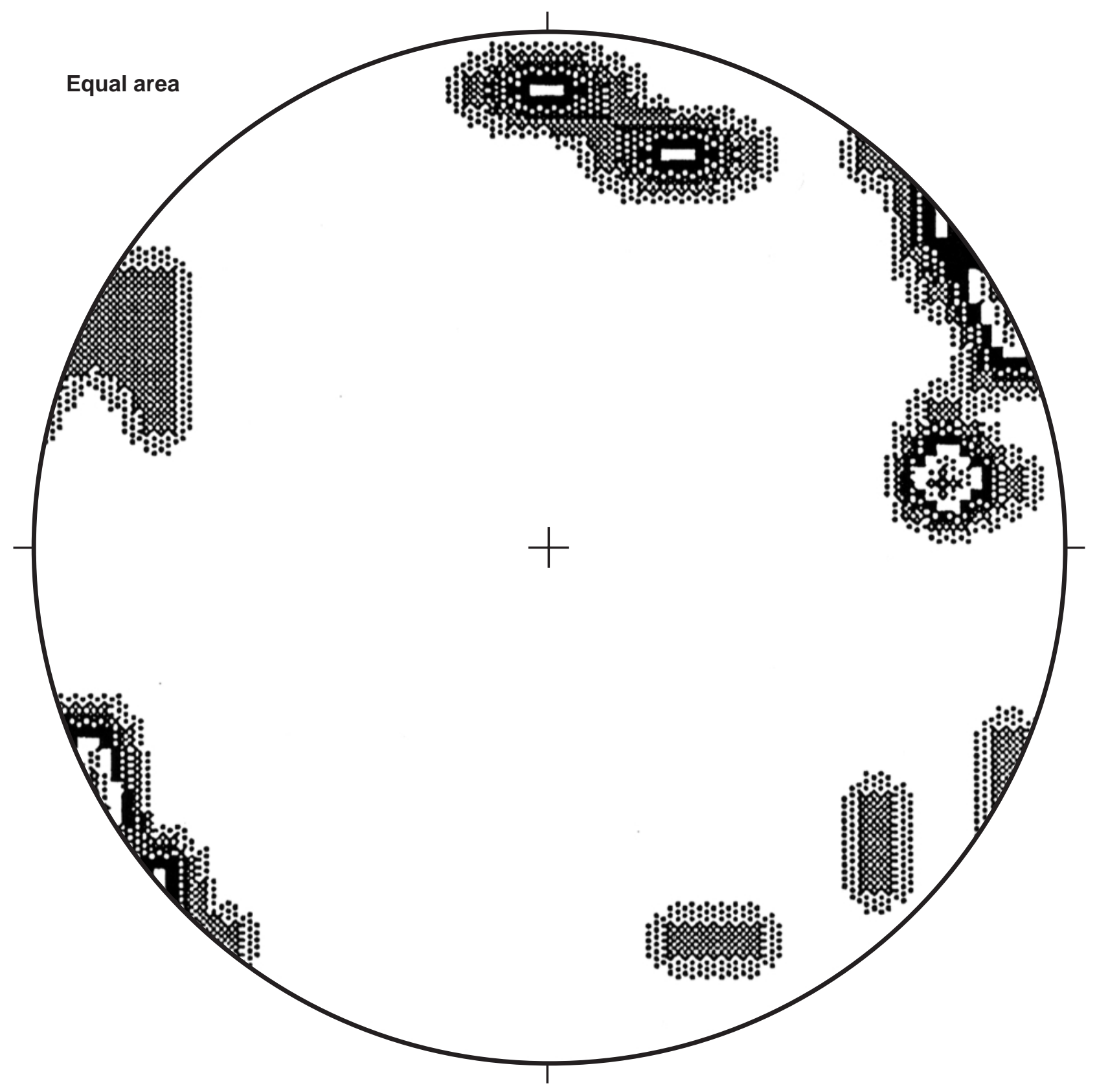

$1 \%$ area contour:

$\mathrm{N}=17 ;$ contour interval $=2 \% / 1 \%$ area

Figure 11. Analysis of fractures mapped after the top surface was leveled above the large block (Stereonet 4.9.5a by Richard Allmendinger of Cornell University in Ithaca, New York) (Wilder, et al., 1997, Section 2, Figure 2-13) 


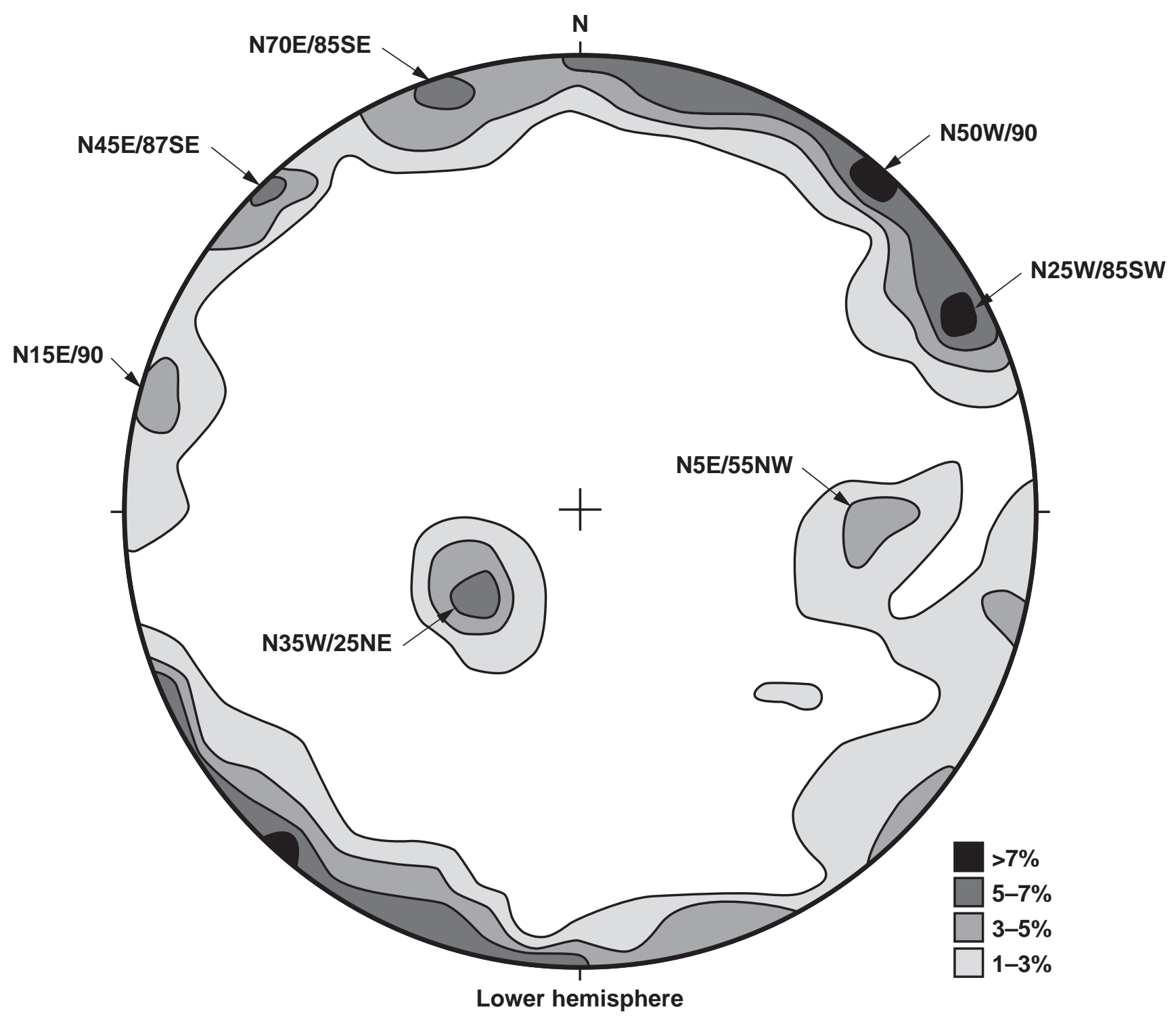

Figure 12. Equal-area contour diagram of poles to 90 fractures mapped in the Climax Stock granite (concentrations in percentage points within one percent area counting circle) (Thorpe and Springer 1981, p. 14, figure 6) 


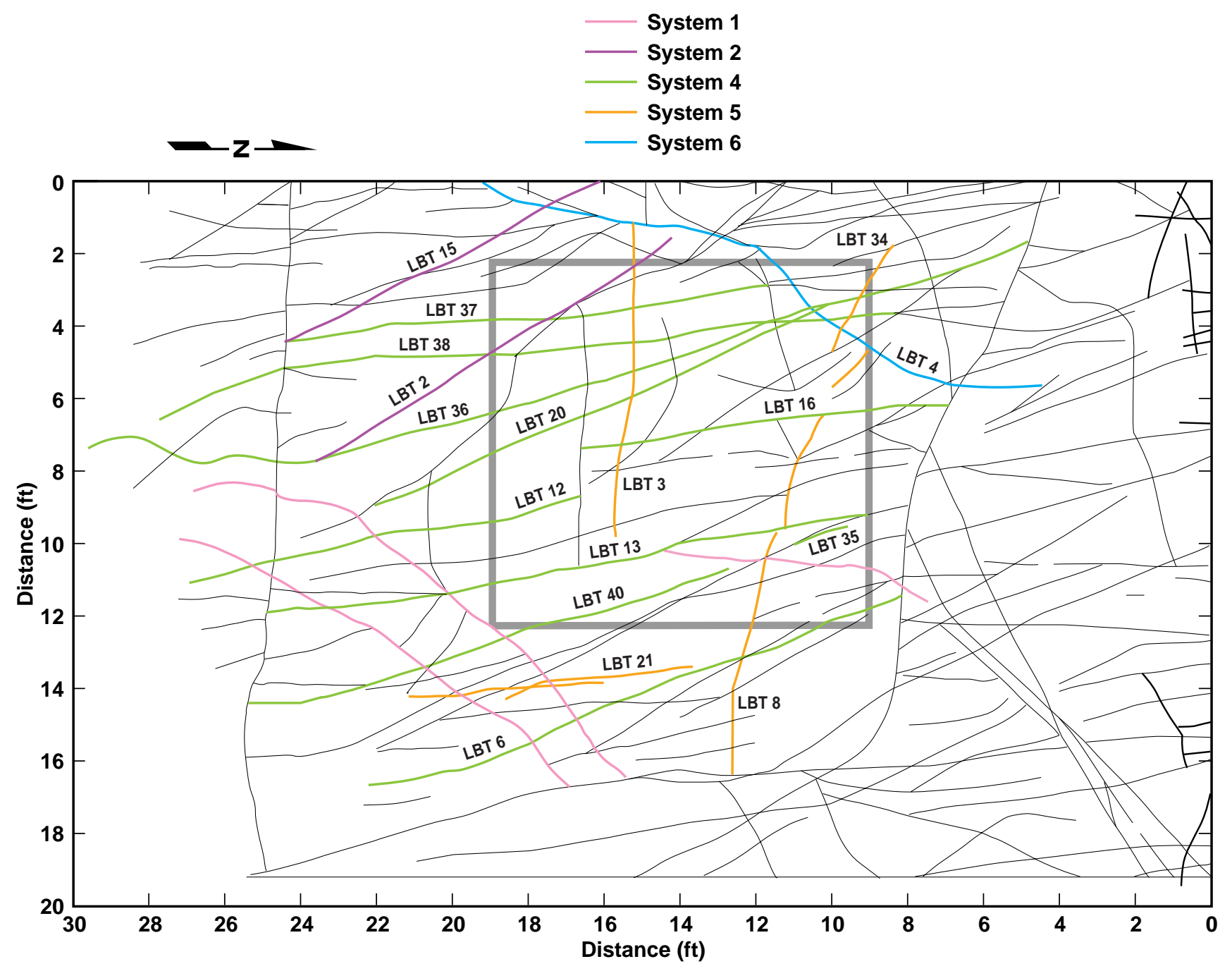

Figure 13. Fractures mapped at the Fran Ridge site prior to construction of the LBT (square): mapped LBT fracture systems were projected to this surface (color coded). (Wilder, et al., 1997, Section 2, Figure 2-1) The gray fractures were not correlated with named fracture surfaces in the block. Also, because of the lack of attitudes for these fractures, the gray fractures could not be specifically assigned to a fracture system. 


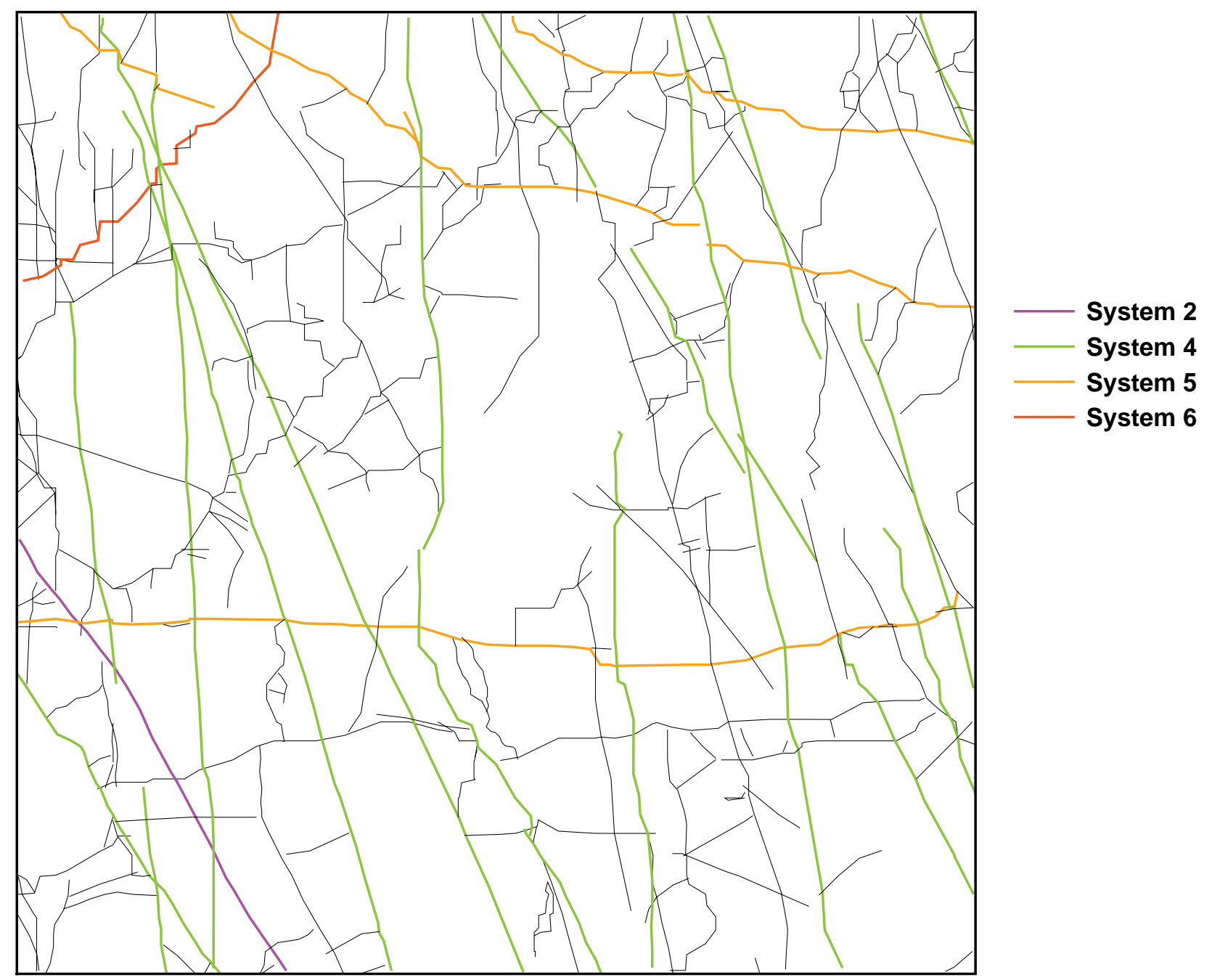

Figure 14. Mapped fractures on top of the LBT 


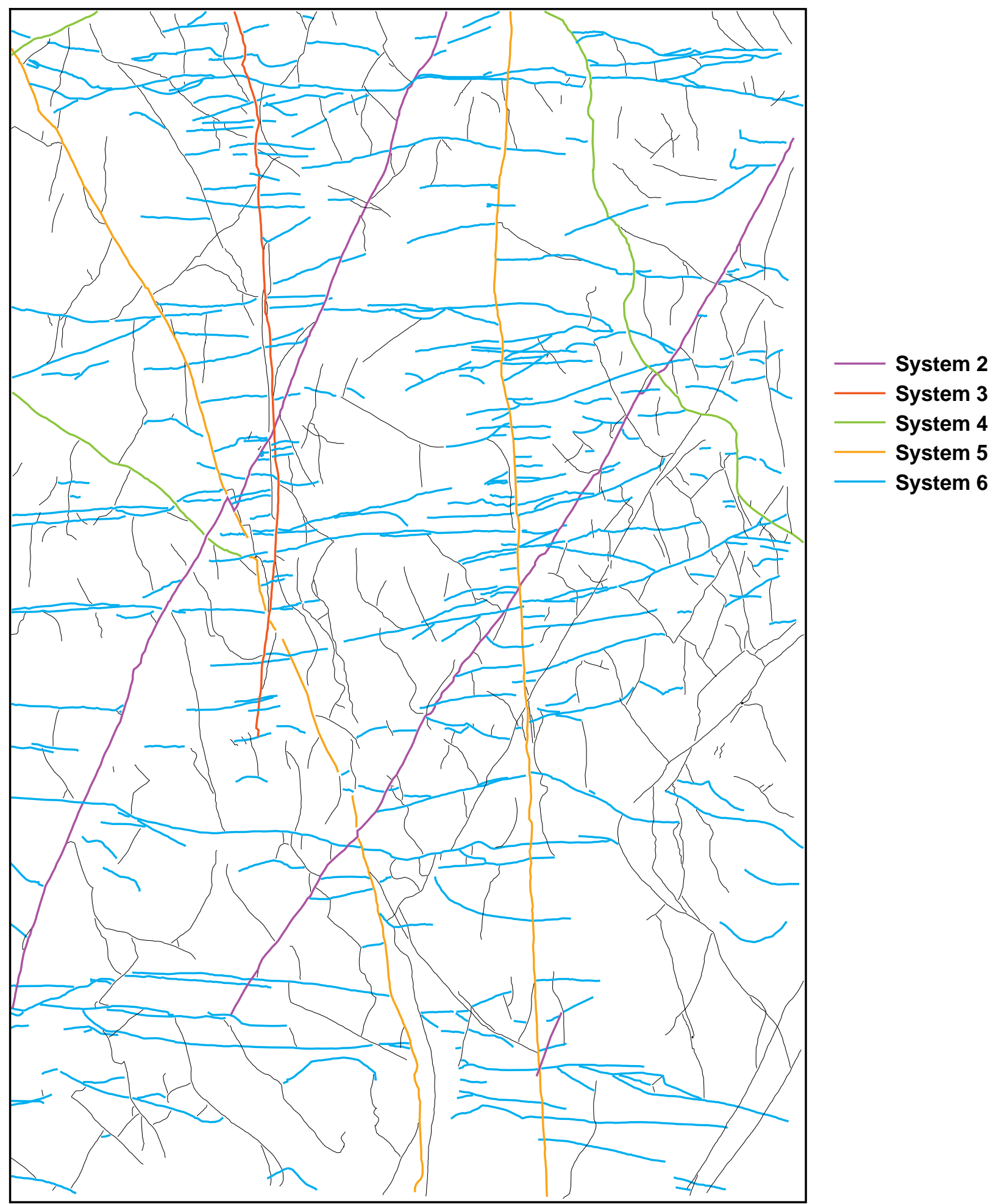

Figure 15. Mapped fractures on the west face of the LBT 


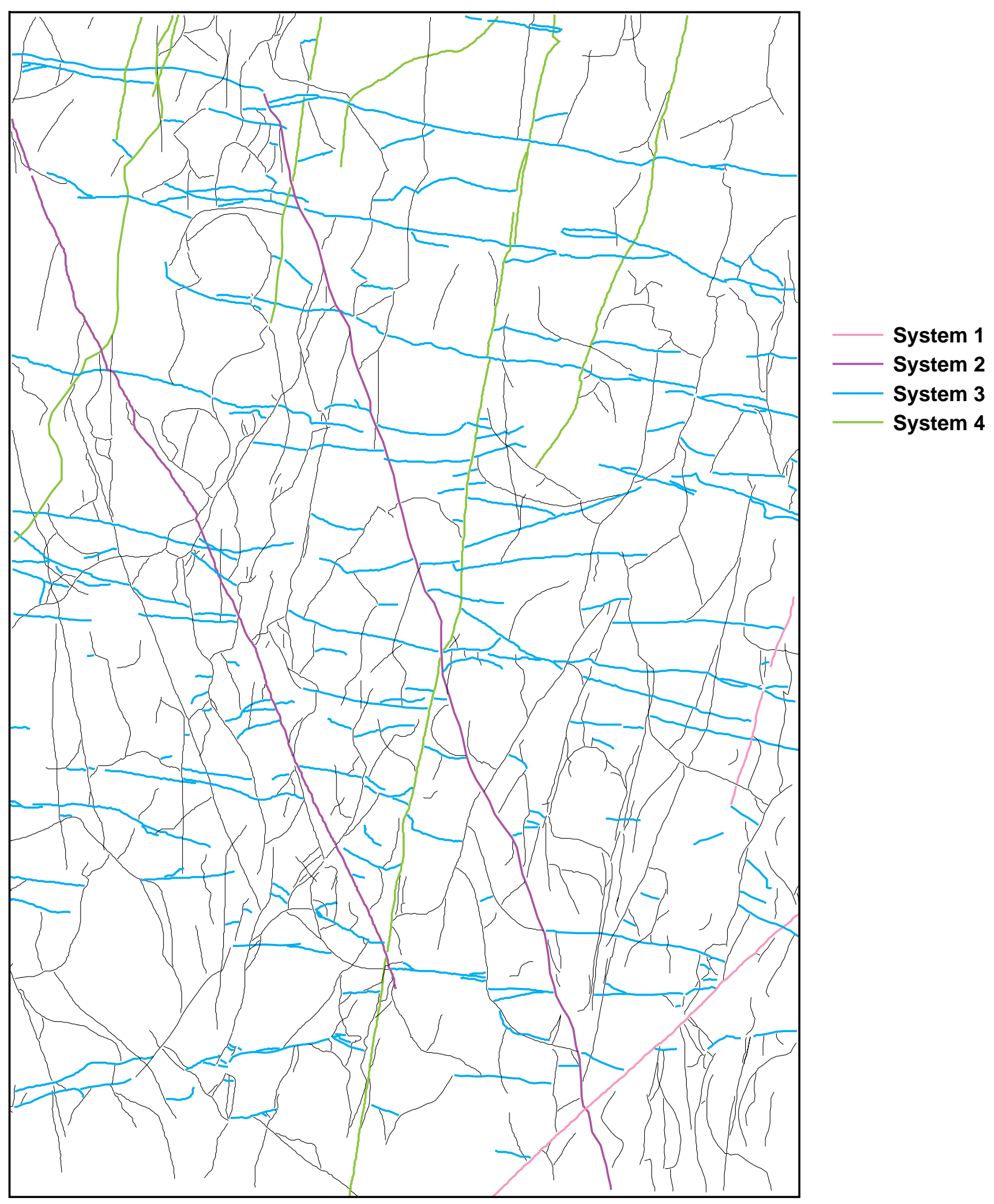

Figure 16. Mapped fractures on the south face of the LBT 


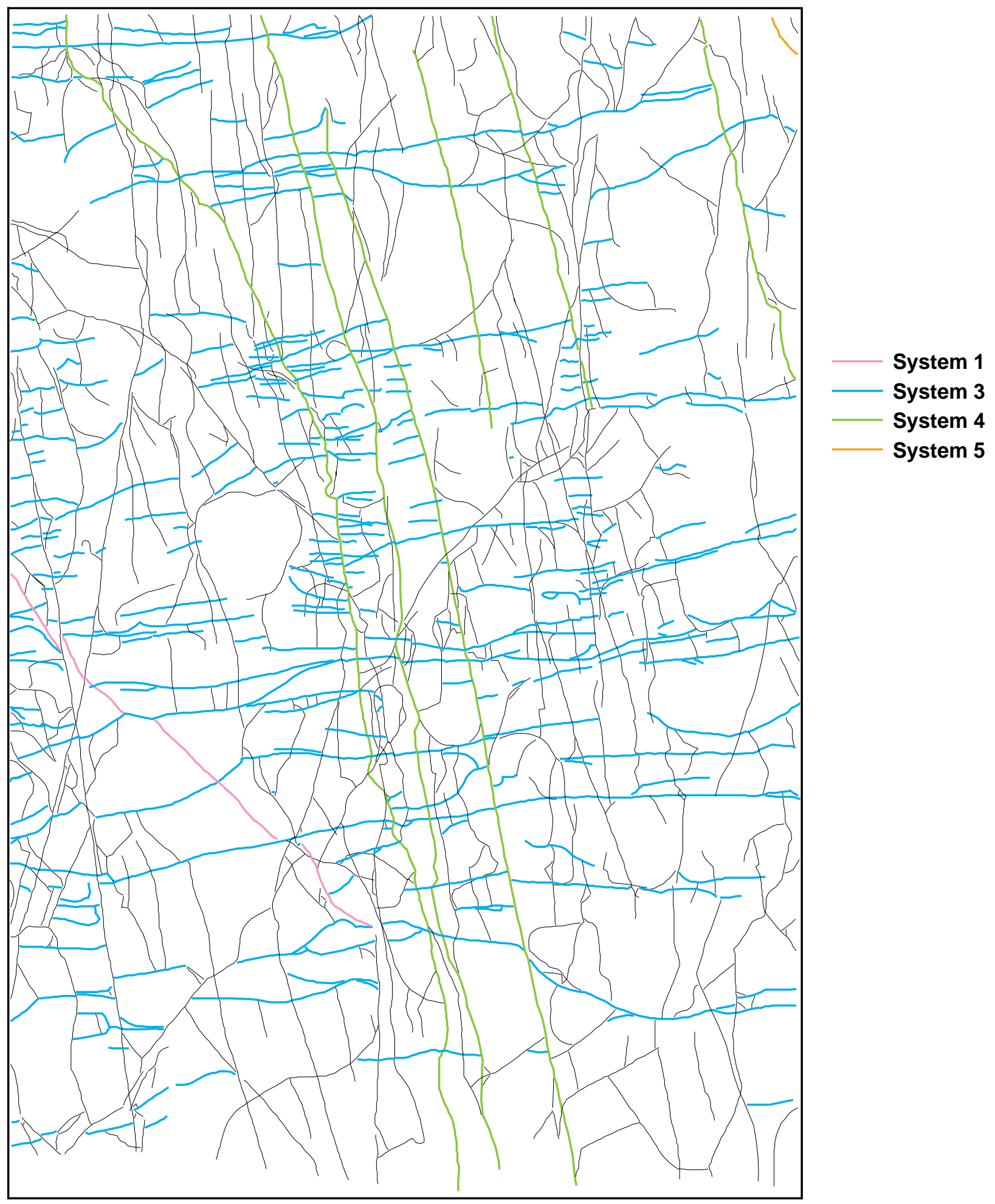

Figure 17. Mapped fractures on the north face of the LBT 


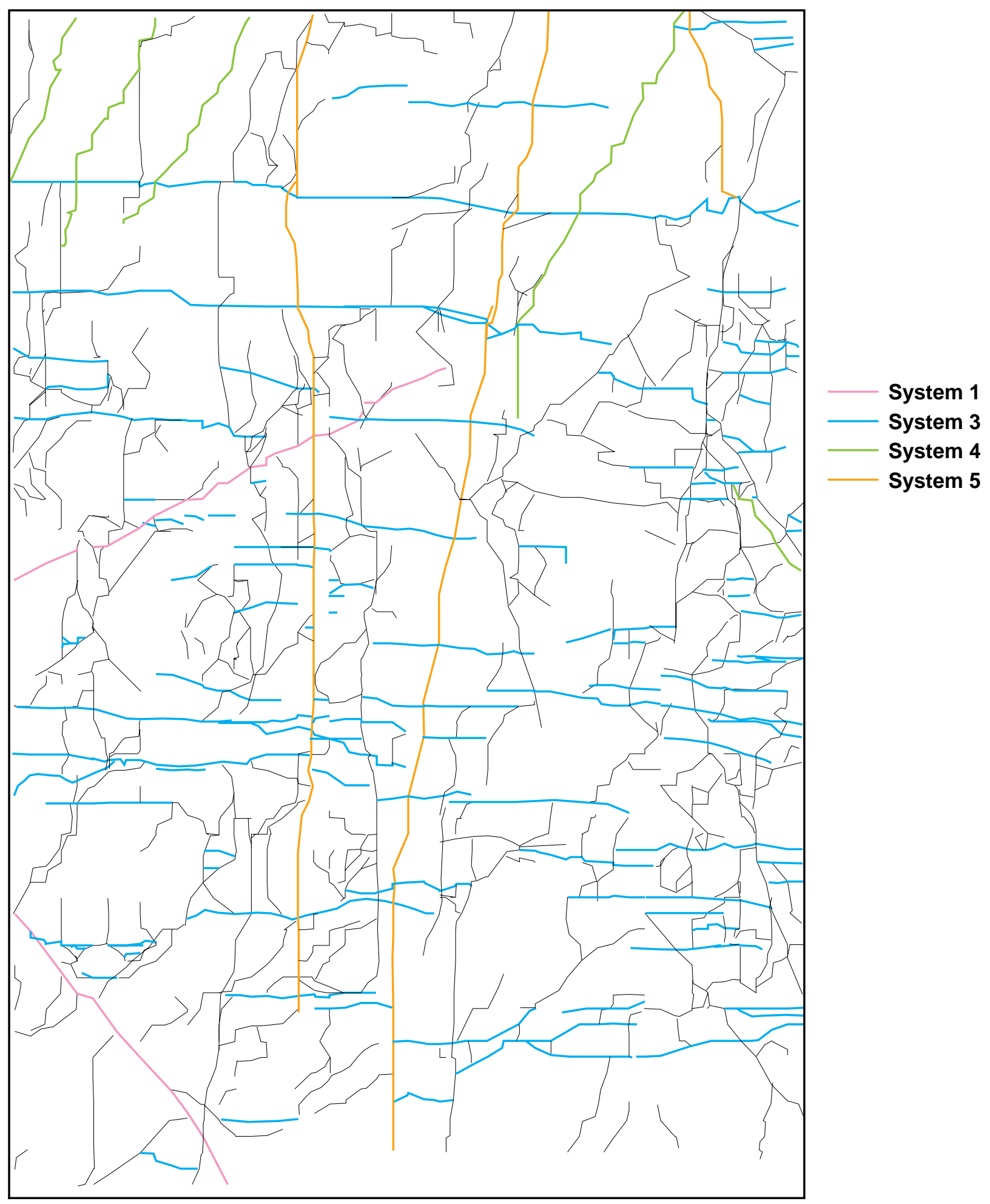

Figure 18. Mapped fractures on the east face of the LBT 